UCRL-ID-130792

\title{
Hydrologic Resources Management Program and Underground Test Area Operable Unit FY 1997 Progress Report
}

\author{
David K. Smith \\ Annie B. Kersting \\ Timothy P. Rose \\ Jacqueline M. Kenneally \\ G. Bryant Hudson \\ Gail F. Eaton \\ M. Lee Davisson
}

May, 1998

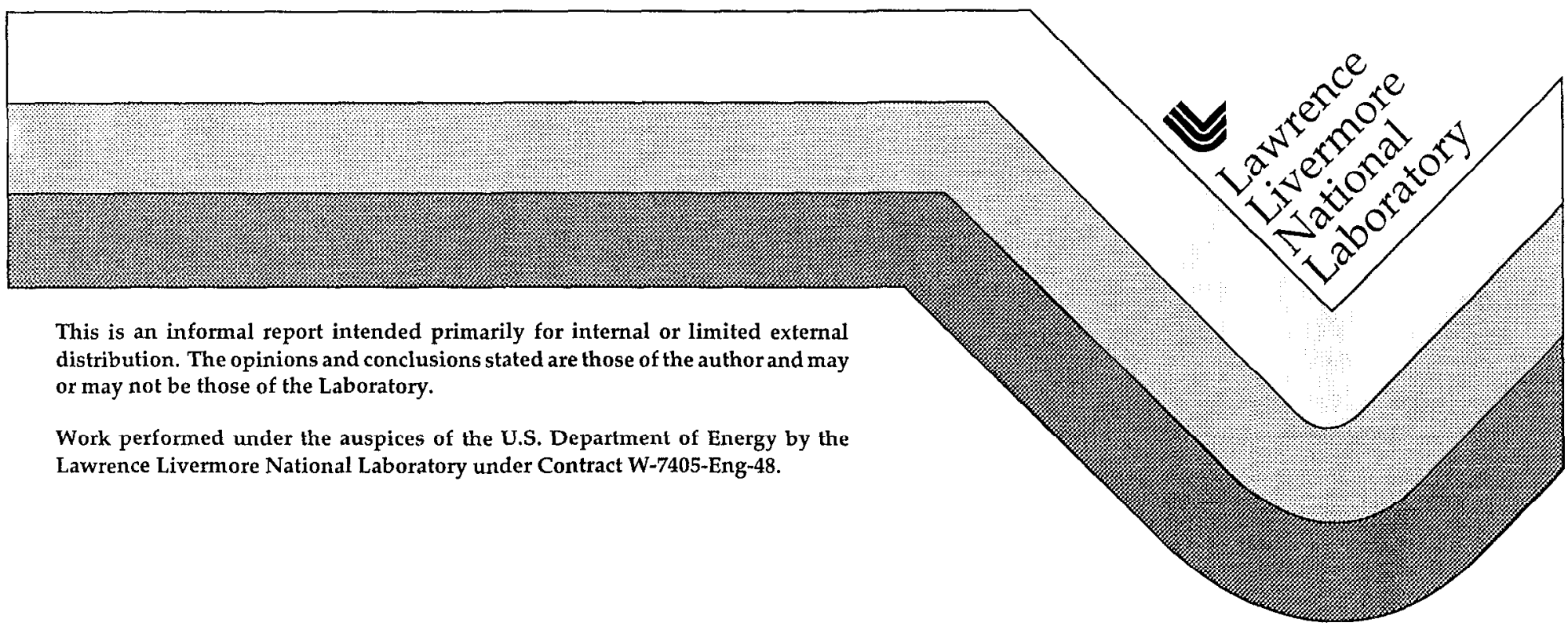




\section{DISCLAIMER}

This document was prepared as an account of work sponsored by an agency of the United States Government. Neither the United States Government nor the University of California nor any of their employees, makes any warranty, express or implied, or assumes any legal liability or responsibility for the accuracy, completeness, or usefulness of any information, apparatus, product, or process disclosed, or represents that its use would not infringe privately owned rights. Reference herein to any specific commercial product, process, or service by trade name, trademark, manufacturer, or otherwise, does not necessarily constitute or imply its endorsement, recommendation, or favoring by the United States Government or the University of California. The views and opinions of authors expressed herein do not necessarily state or reflect those of the United States Government or the University of California, and shall not be used for advertising or product endorsement purposes.

This report has been reproduced

directly from the best available copy.

Available to DOE and DOE contractors from the

Office of Scientific and Technical Information

P.O. Box 62, Oak Ridge, TN 37831

Prices available from (423) 576-8401

Available to the public from the

National Technical Information Service

U.S. Department of Commerce

5285 Port Royal Rd.

Springfield, VA 22161 


\title{
Hydrologic Resources Management Program and Underground Test Area Operable Unit FY 1997 Progress Report
}

\author{
David K. Smith \\ Annie B. Kersting \\ Timothy P. Rose \\ Jacqueline M. Kenneally \\ G. Bryant Hudson \\ Gail F. Eaton \\ M. Lee Davisson
}

May, 1998 



\section{TABLE OF CONTENTS}

Introduction

Chapter 1 Study of the Radiological Situation at the Atolls of Mururoa and Fangataufa

Chapter 2 Analysis of Natural Gas Samples Adjacent to the 1969 RULISON Underground Nuclear test, Grand Junction, Colorado

Chapter 3 Cavity or Near-cavity "Hot" Well Data: FY97

Chapter 4 Older Cavity or Near-cavity “Hot” Well Data: FY77 - FY96

Chapter 5 Batch Leaching of Nuclear Explosive Melt Debris

Chapter 6 Isotope Hydrology Investigation of Regional Groundwater Flow in Central Nevada

Chapter 7 Migration of Plutonium in Groundwater at the Nevada Test Site 


\section{INTRODUCTION}

This report presents the results of FY 1997 technical studies conducted by the Lawrence Livermore National Laboratory (LLNL) as part of the Hydrology and Radionuclide Migration Program (HRMP) and Underground Test Area Operable Unit (UGTA). The HRMP is sponsored by Defense Programs of the U.S. Department of Energy, Nevada Operations Office (DOE/NV) to assess the environmental (radiochemical and hydrologic) consequences of underground nuclear weapons testing at the Nevada Test Site (NTS); other participating organizations include the Los Alamos National Laboratory (LANL), the U.S. Geological Survey (USGS), the United States Environmental Protection Agency (EPA), the Desert Research Institute (DRI) of the University of Nevada, and BechtelNevada (BN). UGTA is sponsored by the Environmental Management Program of DOE/NV as part of a Federal Facilities Agreement and Consent Order between the Department of Energy, the Department of Defense, and the State of Nevada to address the release of hazardous constituents at the Nevada Test Site, Tonopah Test Range, and offNevada Test Site underground testing areas. Participating organizations include LLNL (Earth Sciences and Isotope Sciences Divisions), LANL, DRI, BN, HSI-GeoTrans, and IT Corporation.

The FY97 report represents a departure from the HRMP annual reports issued by LLNL for FY96, FY95 and earlier years. Discussions between DOE/NV, LLNL, and LANL during FY97 indicated the need to produce a single publication from each laboratory that would report all work relating to radionuclide transport at the Nevada Test Site. For this reason, and because some tasks which were historically a part of HRMP are presently supported by UGTA, the national laboratories will now report on results derivative from both FY97 HRMP and UGTA investigations in a single document published by each laboratory.

FY97 was a transitional year for HRMP. The traditional role of this program has been to protect groundwater resources from the consequences of underground nuclear weapons testing. That mission is under increased scrutiny now that U.S. testing has ended. During FY97, discussions between DOE/NV, LLNL, and LANL on the future of HRMP culminated in a presentation to the DOE/NV manager and assistant manager staff in October, 1997 entitled "Future Roles for Radiochemistry and HRMP Five Years After Nuclear Testing". A consensus is that HRMP needs to re-evaluate and possibly re-define its mission to provide strong linkage with the current needs of Defense Programs at DOE/NV. LLNL maintains that HRMP provides an important capability to collect and intrepret data important to the science-based stockpile stewardship program as well as a technical resource for credible stewardship of NTS environmental resources in the context of Defense Programs. The rolc of HRMP in face of changes in the DOE science-based 
stockpile stewardship program, Defense Programs at DOE/NV, and LLNL-Nevada programs will continue to be formulated in FY98.

FY97 tasking focused on radionuclide transport adjacent to sites of underground nuclear tests (i.e., the near-field). This report describes LLNL's participation in an International Atomic Energy Agency sponsored investigation of the radiological consequences of underground nuclear testing by the French government on the atolls or Mururoa and Fangataufa in French Polynesia. LLNL reports measurements of radionuclide concentrations in natural gas samples taken in the vicinity of the 1969 RULISON underground nuclear test fired near Grand Junction, Colorado to assess the potential migration of gaseous radionuclides. LLNL further compiles fluid data collected from or adjacent to saturated nuclear test cavities ("hot" wells) at the CHESHIRE (U-20n) and DALHART (U-4u) events in July, 1997; previously published archived "hot" well analyses are also tabulated. Results of batch leaching experiments of vitreous and crystalline nuclear explosive melt debris in dilute bicarbonate waters from the Nevada Test Site are reported relative to controls on the release of radionuclides to solution. The progress of on-going studies of regional groundwater flow systems using environmental isotopes is provided in the context of a specific investigation of the Railroad Valley flow-system which is proposed as part of a deep Paleozoic fractured rock aquifer which connects sources of precipitation and high elevation recharge north of the Nevada Test Site to flow systems beneath Pahute Mesa. Finally, this report describes results of a collaborative study with LANL to investigate the occurrence of Pu detected in the ER-20-5 \#1 and ER-20-5 \#3 wells which has migrated 1300 meters downgradient the BENHAM (U-20c) explosion beneath Pahute Mesa. The migration of plutonium and actinides under saturated and partially saturated conditions is a principal focus of our FY97 and FY98 technical program.

Besides topical investigations described in the following report, LLNL participated in other activities which supported DOE/NV initiatives in radiochemistry and radionuclide migration. These activities are not further developed in this report but constituted a significant portion of the FY97 effort for LLNL. Among these was a collaboration with LANL, DOE/NV, and other contractors to develop and teach a one-day short course on the occurrence, mobility, and heath effects of radionuclides at the Nevada Test Site. The curriculum was designed to acquaint a general audience to the origin of radioactive material, mechanisms of transport in groundwater, risks associated with groundwater contamination by radionuclides, and strategies planned for corrective actions and remediation. This allday course was presented on September 18, 1997 to 43 DOE/NV affiliated personnel. In addition LLNL was asked by representatives of the American Chemical Society to organize a special session entitled "Investigations of Radioactive and Nonradioactive Source Terms On and Off the Nevada Test Site" at the 214th National Meeting of the American Chemical Society held in Las Vegas from September 7 to 11, 1997. DOE and contractor personnel 
representing defense programs, environmental management, and waste management were invited to participate in this session. LLNL also presented a paper summarizing FY94 to FY97 near-field drilling results for the Nevada Test Site at the Fourth International Conference on Methods and Applications of Radioanalytical Chemistry held from April 6 to 11 in Kailua-Kona, Hawaii. A proceedings paper from this conference entitled "A Recent Drilling Program to Investigate Radionuclide Migration at the Nevada Test Site" (UCRLJC-127013) will be published in FY98 in the Journal of Radioanalytical and Nuclear Chemistry (Smith, 1998, in press). 


\title{
CHAPTER 1
}

\section{STUDY OF THE RADIOLOGICAL SITUATION AT THE ATOLLS OF MURUROA AND FANGATAUFA}

\author{
David K. Smith
}

Following the cessation of nuclear testing on the atolls of Mururoa and Fangataufa in French Polynesia in 1996, the International Atomic Energy Agency (IAEA) was asked by the French Government to conduct an independent study of the radiological impact of testing on potentially affected receptor populations throughout the South Pacific region. In June, 1996, the "Study of the Radiological Situation on the Atolls of Mururoa and Fangataufa" was organized by the IAEA to conduct this investigation. The scope of the study is two-fold and comprises an evaluation of current radiological conditions as well as an evaluation of the potential long term radiological situation at the Centre d'Expérimentations du Pacifique (CEP). The CEP includes both the Mururoa and Fangataufa atoll testing areas. In order to execute this study, an International Advisory Group has been appointed which consists of experts in nuclear radiation and health physics from Argentina, Australia, Germany, Indonesia, Japan, New Zealand, the Russian Federation, Sweden, the United Kingdom and the United States. Two Task Groups support an International Advisory Committee which includes experts who provide guidance and direction to the IAEA on the conduct of the Study. Task Group A is charged with evaluating the current extent of radiological contamination and Task Group B is charged with evaluating the potential for the long term release and transport of radionuclides. Each Task Group is in turn supported by Working Groups with technical expertise in terrestrial contamination, aquatic contamination, the underground radionuclide inventory, radionuclide release and transport, lagoon/open-ocean circulation and dispersion, and marine modeling. The IAEA is due to publish its final report in July, 1998.

Because of LLNL's experience in the design and execution of programs to assess the migration of radionuclides residual from underground nuclear weapons testing at the Nevada Test Site (NTS), the IAEA invited LLNL staff (D. Smith) to participate in this study through membership in Task Group B which is concerned with the long-term consequences of nuclear testing on the atolls. In addition to membership on Task Group B, LLNL collaborated closely with Working Group 3 on the definition of a radiologic source term, and Working Group 4 on modeling radionuclide transport beneath the atolls. LLNL also played a principal role in the organization and execution of a sampling campaign to collect water from monitoring and cavity wells beneath Mururoa and Fangataufa.

Mururoa and Fangalaufa are both volcanic atolls, capped by carbonate, and coral, situated approximately $1000 \mathrm{~km}$ southwest of Tahiti in the Tuamotu Archipelago. The French have conducted a total of 137 underground nuclear tests, 41 atmospheric tests, and 15 safety 
experiments at the CEP during the period 1966 to 1996. Figure 1 is a map of all underground nuclear tests (with nuclear yields in kilotons listed by range) fired on the Mururoa atoll.

The design of the IAEA study is to determine a dose assessment to potential receptors through independent expert investigation supplemented by data provided by the French Government. The structure of this investigation utilizes numerical models of water flow and contaminant transport which input data to calculate a dose assessment. Data requirements included information on the atoll geology, geologic pathways (fractures and faults), atoll hydrology, hydraulic conductivity, the radiologic source term residual from the underground explosions (including tritium, fission products, activation products, and residual actinides), the solution and sorption of radionuclides derived from debris produced during the explosions, radionuclide releases to the overlying lagoons and open ocean, and models of marine transport. To independently verify historical data returned by the French Government as well as to provide data to validate modeling efforts, the IAEA planned a sampling campaign on the atolls to collect fluids from a network of monitoring wells and wells completed in detonation cavities created by the nuclear explosions. In collaboration with the IAEA, the French military, and the Commissariat a l'Energie Atomique (CEA), LLNL helped organize and conduct the sampling program on the atolls. The underground sampling provides an cmpirical measure of the solution source which is composed of radionuclides dissolved in or otherwise available for transport by water circulating through the atolls. The sampling campaign ultimately provides an objective measure of potential individual and collective dose of particular interest to populations in French Polynesia and throughout the Pacific Rim. Final results will be presented at an IAEA-sponsored international conference in July, 1998.

LLNL's participation in the Study consisted of attendance at three meetings of Task Group B in Paris, France and Vienna, Austria over the period July, 1996 to August, 1997 as well as organization of an underground sampling campaign to sample eleven wells beneath the rim and lagoon of Mururoa and Fangataufa atolls during a two week period in May and June, 1997. Besides sharing U.S. experience on the occurrence, distribution, and transport of radionuclides associated with underground nuclear testing with the IAEA, LLNL, HRMP, and UGTA also benefited through collaboration with recognized international experts in the fields of geology, hydrology, rock mechanics, radiochemistry, nonproliferation and arms control, and numerical modeling relative to formulating comprehensive estimates of radionuclide release and dose assessment associated with underground nuclear testing.

A detailed discussion of the organization, approach, and results of the Study is outside the scope of this report. Interested parties are referred to the final Study Report and derivative 
technical reports to be published by the IAEA in the spring of 1998. Further questions about the Study, its findings, or final reports should be directed to LLNL-HRMP. 
337 underground tests (satety tests induded)

Total Yeid (Wtot) -2.4 megatons (MT)

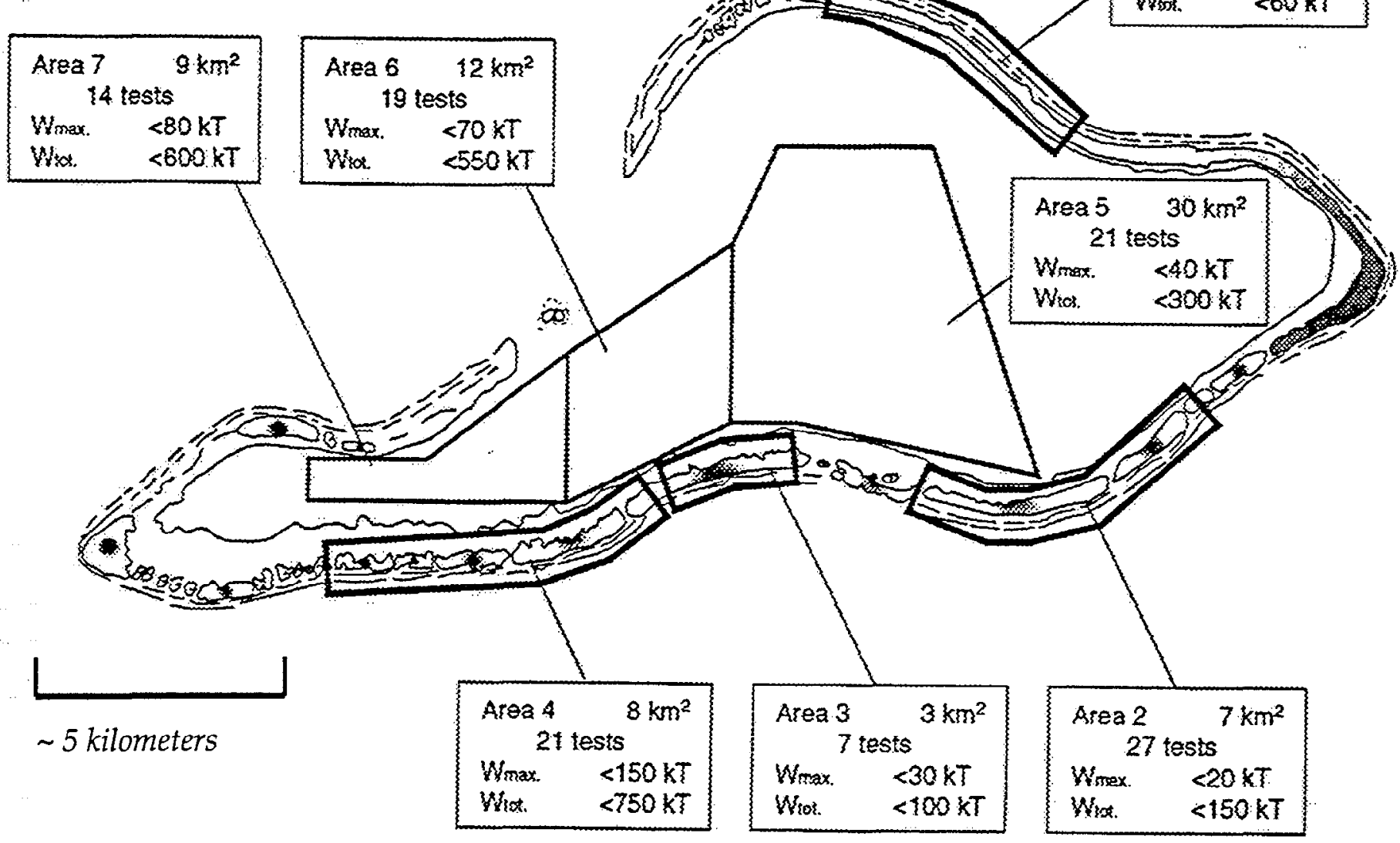

Figure 1: Map of Mururoa atoll showing underground testing areas and number of tests within each. Yield in kilotons expressed as W. Note testing areas included both atoll rim and lagoon. 


\title{
CHAPTER 2
}

\section{ANALYSIS OF NATURAL GAS SAMPLES ADJACENT TO THE 1969 RULISON UNDERGROUND NUCLEAR TEST, GRAND JUNCTION, COLORADO}

\author{
G. Bryant Hudson
}

LLNL received a request in 1997 to analyze five natural gas samples collected from gas wells within several kilometers of the RULISON underground nuclear test fired in September, 1969 between Grand Junction and Rifle, Colorado. This 43 kiloton yield offNevada Test Site nuclear test was part of joint project between the Atomic Energy Commission and the Austral Oil Company to assess the feasibility of using nuclear explosives to stimulate production from tight (less transmissive) natural gas fields. Recent interest in increasing the number of natural gas production wells in this area culminated in a priority request that the U.S. Department, Nevada Operations Office evaluate how close gas wells could be drilled to the RULISON ground zero without radioactivity residual from the explosion contaminating the gas produced from these wells. The gas samples consisted primarily of methane $\left(\mathrm{CH}_{4}\right)$ with less amounts of ethane, propane, and $\mathrm{CO}_{2}$. There are many gaseous fission (and activation) products produced during a nuclear cxplosion including ${ }^{3} \mathrm{H},{ }^{14} \mathrm{C},{ }^{37} \mathrm{Ar},{ }^{39} \mathrm{Ar},{ }^{85} \mathrm{Kr}$, and ${ }^{133} \mathrm{Xe}$ - that might substitute in the molecular structure (in the case of $\mathrm{C}$ and $\mathrm{H}$ ) or accompany the hydrocarbon molecules. Of these ${ }^{3} \mathrm{H}$, ${ }^{14} \mathrm{C}$, and ${ }^{85} \mathrm{Kr}$ are sufficiently long lived $\left(\mathrm{t}_{1 / 2}>10\right.$ years) that they might possibly be detected in the nearly thirty-years since the detonation. Their presence in the regional gas wells was not expected due to inherent fractionation and dispersion of these radionuclides.

Initial screening of the samples by thin-window $\beta$ counting indicated no activity was present $(<200 \mathrm{dpm} / \mathrm{L})$. Samples were counted by gas flow proportional counting which is particularly well suited in this case because the gas could be used directly in the counters without separation or pre-concentration. Methane blanks were measured along with the unknowns. Each sample was counted 4 times (except RUG00001 which was analyzed three times). Each sample was analyzed using at least two different sampling tubes. Results are presented in Table I. The results do not discriminate between ${ }^{3} \mathrm{H},{ }^{14} \mathrm{C}$, and ${ }^{85} \mathrm{Kr}$; reported activities are the total for all gaseous radionuclides present. The uncertainity represents a $2 \sigma(95 \%)$ confidence interval for the weighted average for all analyses for each sample. Total activities are reported in $\mathrm{pCi} / \mathrm{L}$ and $\mathrm{Bq} / \mathrm{L}$ at the time of counting. A complete discussion of analytical procedures and results are contained in a LLNL letter report to DOE/NV (Hudson, 1997). 


\section{TABLE I}

${ }^{3} \mathrm{H},{ }^{14} \mathrm{C}$, and ${ }^{85} \mathrm{Kr}$ in Natural Gas Samples Adjacent to the RULISON Event

\begin{tabular}{lllll}
\hline SAMPLE & $\begin{array}{l}\text { Total Activity } \\
(\mathrm{pCi} / \mathrm{L})\end{array}$ & $\begin{array}{l}\text { Total Activity } \\
(\mathrm{Bq} / \mathrm{L})\end{array}$ & $\begin{array}{l}\text { Error }(2 \sigma) \\
(\mathrm{pCi} / \mathrm{L})\end{array}$ & $\begin{array}{l}\text { Error }(2 \sigma) \\
(\mathrm{Bq} / \mathrm{L})\end{array}$ \\
\hline & & & & \\
RUG00001 & 7 & $2.6 \mathrm{E}-1$ & 42 & 1.6 \\
RUG00002 & -1 & $-3.7 \mathrm{E}-2$ & 27 & 1 \\
RUG00003 & 1 & $3.7 \mathrm{E}-2$ & 27 & 1 \\
RUG00004 & -7 & $-2.6 \mathrm{E}-1$ & 27 & 1 \\
RUG00005 & 15 & $5.6 \mathrm{E}-1$ & 27 & 1 \\
\hline
\end{tabular}

These results suggest production areas are not compromised by gaseous radionuclides derived from the RULISON event.

\section{REFERENCES}

Hudson, G.B., 1997, Letter report to Environmental Restoration Division, U.S. Department of Energy, Nevada Operations Office from Isotope Sciences Division I awrence Livermore National Laboratory. 


\title{
CHAPTER 3
}

\section{CAVITY OR NEAR-CAVITY "HOT" WELL DATA: FY97}

\author{
Jacqueline M. Kenneally
}

To understand better the hydrologic source term and potential radionulcide migration away from the sites of underground nuclear explosions, LANL and LLNL have collected and analyzed fluid samples annually from post-shot drill holes and other near-cavity wells (e.g., Marsh, 1992 and Thompson, 1998). Figure 1 is a map of drill-holes and wells historically investigated by HRMP and UGTA. In recent years, sample collection has been restricted to bailing at discrete intervals. Bailing relies on a down-hole wireline capability which was transferred from the Joint Test Organization (JTO) to the USGS in FY97. The FY97 sampling campaign successfully demonstrated the continued ability to collect discrete samples.

During FY97 scientists from the USGS, LANL, and LLNL collected fluid samples from post-shot drill holes at U-20n PS\#1DD-h (CHESHIRE) and U-4u PS2a (DALHART). One sample was collected from a single interval at U-4u PS2a and four samples were collected from separate depth intervals at U-20n PS\#1DD-h. In past years these wells have been sampled at similar depths (see Smith et al., 1997). Repeated sampling creates a baseline against which temporal variations in radionuclide concentrations may be compared. Analyses of radionuclides from these two sites are particularly significant since both holes are proposed for rehabilitation and pumping in FY98.

\section{U-20n PS\#1DD-h (CHESHIRE)}

Well U-20n PS\#1DD-h is a post-shot hole that was drilled to collect diagnostic radiochemical core samples following the February 14, 1976 CHESHIRE event. Subsequently, well U-20n PS\#1DD-h was pumped both within and above the cavity as part of a comprehensive radionuclide migration study in fractured volcanic rocks (e.g., Buddemeier and Isherwood, 1985). Pumping allows production of fluids which are more representative of formation water. Pumping above the cavity was suspended in 1985. All samples collected since that time have been bailed.

Results from samples collected from U-20n PS\#1DD-h in July, 1997 are listed in Table 1 and are consistent with previous analyses (see Tables 2 through 4 in Chapter 4). The ${ }^{3} \mathrm{H}$ results listed in Table 1 are field measurements with a higher uncertainty than analyses conducted in the laboratory. Despite the higher uncertainty, activity levels are comparable with recent measurements. Not unexpectedly, analytical results from samples collected during the 1970's and 1980's indicate the waters produced by pumping the CHESHIRE 
cavity contained much higher levels of activity than those taken from above the cavity (e.g., Buddemeier, 1988; Smith et al., 1997). Comparison between current concentrations of radionuclides found within the cavity and concentrations encountered in 1985 must wait for the proposed recompletion and repumping of the well in FY98.

The depth of the static water level measured in 1997 for well U-20n PS\#1DD-h was 640 meters (2099 feet) below surface which is consistent with previous measurements. This depth represents the measured slant depth; no attempt was made to correct to vertical depth below ground surface.

\section{U-4u PS2a (DALHART)}

Well U-4u PS2a is the post-shot hole that was drilled back into the DALHART event fired on October 13, 1988. Fluid samples were collected in 1993 at a depth of $533 \mathrm{~m}$ (1748 feet) using evacuated bailers similar in design to the system employed during FY97 (see Smith et al., 1997). The 1993 samples were extensively contaminated with drilling fluid. In 1995, LANL attempted to air lift (jet) water from the hole; unfortunatcly this approach proved unsuccessful despite copious amounts of water added to dilute the drilling fluids (Thompson, 1995). Fluid samples were not collected during the 1995 exercise.

Collection of a representative groundwater sample from this hole is important because low levels of $\mathrm{Pu}$ were detected in the fluid sample collected in $1993\left({ }^{239} \mathrm{Pu}+{ }^{240} \mathrm{Pu}\right.$ : $1.36 \mathrm{E}-01$ $\mathrm{pCi} / \mathrm{L}$ ). Results for the 1997 sample are listed in Table 1. Pu was again detected in 1997 $\left({ }^{239} \mathrm{Pu}+{ }^{240} \mathrm{Pu}: 1.92 \mathrm{E}-01 \mathrm{pCi} / \mathrm{L}\right)$. This sample was neither filtered nor preserved in the field. Upon return to the laboratory, it was filtered through a $0.45 \mu \mathrm{m}$ filter and both filter and filtrate were analyzed. The $\mathrm{Pu}$ is associated with the solid material trapped on the filter. The significance of transport of Pu via non solute phases at the Nevada Test Site is discussed by Thompson (1998), Kersting et al. (in press), and in Chapter 7 of this report.

The activity levels for other radionuclides, such as ${ }^{60} \mathrm{Co},{ }^{106} \mathrm{Ru},{ }^{125} \mathrm{Sb},{ }^{137} \mathrm{Cs}$ and ${ }^{226} \mathrm{Ra}$, are higher in the 1997 sample compared to samples previously collected (for comparison see Table 3 in Chapter 4). In contrast, LANL notes that the ${ }^{3} \mathrm{H}$ activity of the 1997 sample is approximately $50 \%$ of the 1993 sample and suggests the lower concentrations may be attributable to water introduced into the hole during the 1995 air-lifting exercise (Thompson, 1998). The concentration of major and trace elements is significantly lower in the 1997 sample when compared with the 1993 sample (for comparison see Table 3 in Chapter 4). This could also be the result of dilution by the additional water injected downhole. 
The concurrent decrease in ${ }^{3} \mathrm{H}$ concentration and increase in other radionuclides between 1993 and 1997 is curious. Previous work has shown that fluids sampled at different depths in the same well bore can have different activity levels due to poor fluid circulation (see Tables 2 through 4 in Chapter 4). Radionuclide concentrations in bailed fluid samples are not necessarily representative of concentrations in formation water. Therefore, caution should be exercised before interpreting these data due to, 1) known contamination of 1993 samples with drilling mud, 2) addition of large quantities of water into the hole in 1995, and 3) data from only two years $(1993,1997)$.

We speculate that drilling fluids present in 1993 may have been sufficiently disseminated to increase the effective surface area available for reaction with radionuclides dissolved or suspended in the groundwater. Radionuclides, excluding tritium, may have sorbed onto the sediments. This increase in effective surface area may allow more radionuclides to sorb and may explain the increased activity levels detected in the 1997 sample. Similarly, the concentration of dissolved species would appear to decrease in the cavity due to dilution by water introduced from the surface. Until the drilling fluid and water introduced during the 1995 operations are purged from the well and a representative sample of cavity fluid can be collected and analyzed, radionuclide concentrations in the cavity cannot be determined with certainty.

\section{REFERENCES}

Buddemeier, R.W. and D. Isherwood, 1985, Radionuclide Migration Project 1984 Progress Report, Lawrence Livermore National Laboratory, UCRL-53628, pages 6-14.

Buddemeier, R.W., ed., 1988, Hydrology and Radionuclide Migration Program 19851986 progress report, Lawrence Livermore National Laboratory, UCRL-53779, pp. 102.

Kersting, AB, D.W. Efurd, D.L. Finnegan, D.J. Rokop, D.K. Smith, and J.L. Thompson, Migration of plutonium in groundwater at the Nevada Test Site, in press, Nature.

Marsh, K.V., 1991, Hydrology and Radionuclide Migration Program 1987 Progress Report, Lawrence Livermore National Laboratory, UCRL-53779-87, p. 3-6.

Smith, DK, A.B. Kersting, J.M. Kenneally, J.H. Rego, J.L. Thompson, 1997, Hydrologic Resources Management Program FY-1996 Progress Report, UCRL-ID126886, pp. 40.

Thompson, J.L., 1998, Laboratory and Field Studies Related to Radionuclide Migration at the Nevada Test Site. October 1, 1996 - September 30, 1997, Los Alamos National Laboratory, 3419-PR Progress Report, 31p.

Thompson, J.L., 1995, Laboratory and Field Studies Related to the Hydrologic Resources Management Program, Los Alamos National Laboratory, LA-13064-PR Progress Report, $27 \mathrm{p}$. 


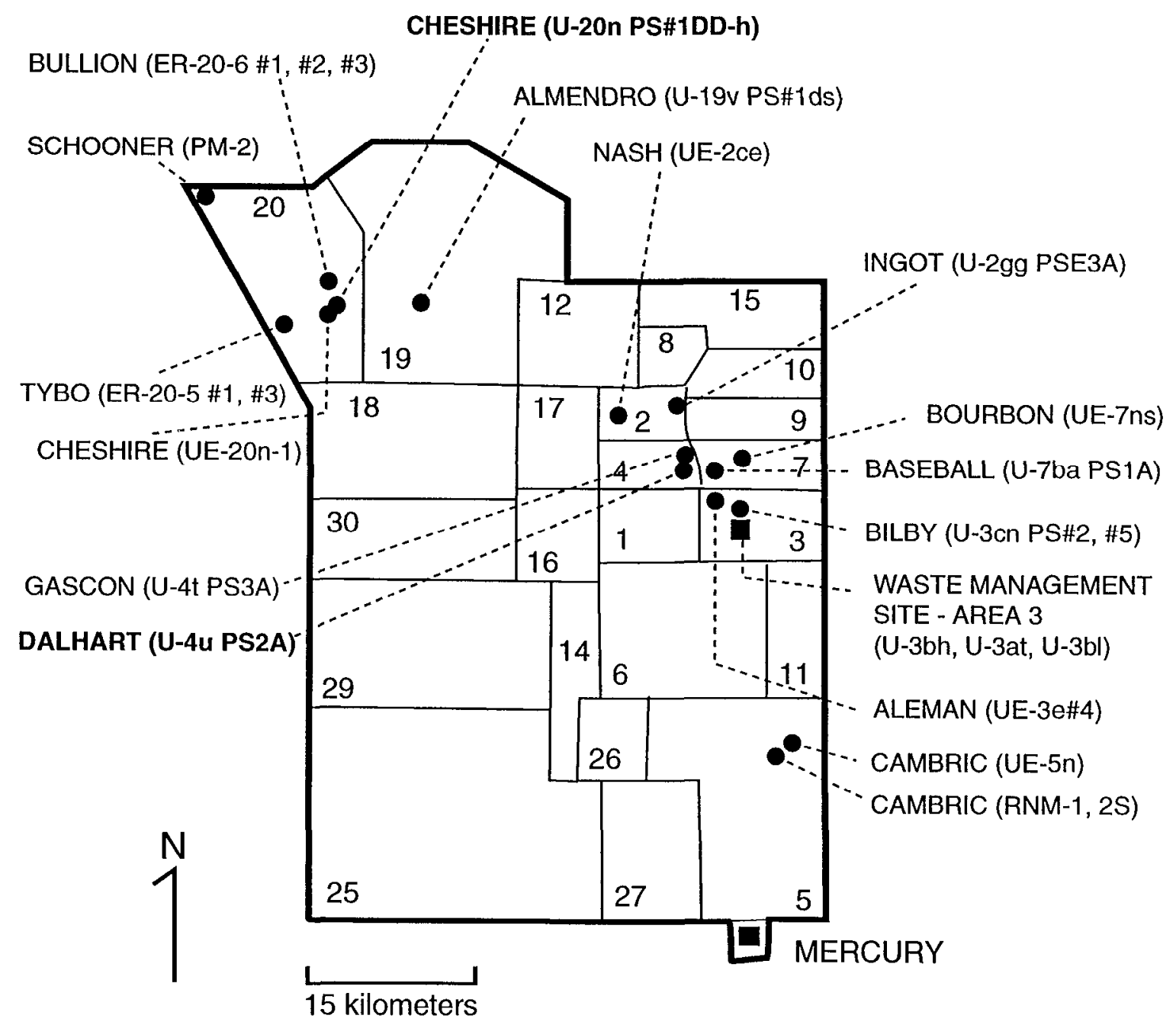

Figure 1: Cavity or near-cavity ("hot") drill-holes and wells studied by the Hydrologic Resources Management Program and Underground Test Area Operable Unit at the Nevada Test Site. Highlighted wells were sampled by wire-line bailer in FY97 as part of the LLNL-LANL-USGS hot well sampling program. 


\section{TABLE I}

\section{Cavity and Near-Cavity "Hot" Well Analyses \\ 1997}

\begin{tabular}{|c|c|c|c|c|c|c|c|c|c|c|c|c|c|c|}
\hline Well name & Event & Depth & $\begin{array}{l}\text { Collection } \\
\text { date }\end{array}$ & ${ }^{3} \mathrm{H}$ & ${ }^{3} \mathrm{H}$ & ${ }^{14} \mathrm{C}$ & $\delta^{13} \mathrm{C}$ & $\delta D$ & $\delta^{18} O$ & ${ }^{60} \mathrm{Co}$ & ${ }^{60} \mathrm{Co}$ & ${ }^{90} \mathrm{Sr}$ & ${ }^{90} \mathrm{Sr}$ & ${ }^{106} \mathrm{Ru}$ \\
\hline Unit & & $(f t)$ & & $(p C i / L)$ & $(p C i / L)$ & pme & $\%$ & $\%$ & $\%$ & $(p C i / L)$ & $(p C i / L)$ & $(\mathrm{pCi} / \mathrm{L})$ & $(\rho C i / L)$ & $(p C i / L)$ \\
\hline Half-life (a) & & & & 12.3 & 12.3 & 5280.0 & stable & stable & stable & 5.3 & 5.3 & 28.8 & 28.8 & 1.0 \\
\hline Ref date & & & & collect. & $1 / 1 / 94$ & collect. & collect. & collect. & collect. & collect. & $1 / 1 / 94$ & collect. & $1 / 1 / 94$ & collect. \\
\hline U-4u PS2a & Dalhart & 1680 & 21-Jul-97 & $1.56 e+7$ & $1.91 €+7$ & $2.49 e+4$ & -8.9 & -107.0 & -12.8 & $1.03 e+1$ & $1.69 e+1$ & $3.11 \theta+0$ & $3.39 e+0$ & $3.27 e+1$ \\
\hline U-20n PS\#1DD-h & Cheshire & 2250 & 22-Jul-97 & $4.53 e+7$ & $5.54 e+7$ & - & - & -120.0 & -14.8 & $(4.07 e-1)$ & $(6.66 e-1)$ & $2.32 \theta+1$ & $2.53 e+1$ & $(3.83 e+0)$ \\
\hline U-20n PS\#1DD-h & Cheshire & 2550 & 22-Jul-97 & $4.48 e+7$ & $5.48 e+7$ &. & - & -121.0 & -14.8 & $(2.35 \mathrm{e}-1)$ & $(3.86 e-1)$ & & 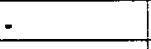 & $(2.09 e+0)$ \\
\hline U-20n PS\#1DD-h & Cheshire & 2650 & 22-Jul-97 & $3.71 e+7$ & $4.53 e+7$ & - & - & .124 .0 & -14.8 & $(4.12 \mathrm{e}-1)$ & $(6.78 e-1)$ & & - & $(3.68 e+0)$ \\
\hline U-20n PS\#1DD-h & Cheshire & 2750 & 23-Jul-97 & $2.73 e+7$ & $3.34 e+7$ & $2.97 e+4$ & -6.0 & -124.0 & -15.0 & $(2.66 e-1)$ & $(4.38 e-1)$ & - & - & $(2.20 e+0)$ \\
\hline \multicolumn{2}{|c|}{ NOTE: ()$=$ detection limit. } & & & & & & & & & & & & & \\
\hline \multicolumn{4}{|c|}{.$=$ analysis not completed on this sample. } & & & & & & & & & & & \\
\hline
\end{tabular}

\begin{tabular}{|c|c|c|c|c|c|c|c|c|c|c|c|c|c|}
\hline Well name & Event & Depth & ${ }^{106} \mathrm{Ru}$ & ${ }^{125} \mathrm{Sb}$ & ${ }^{125} \mathrm{Sb}$ & ${ }^{137} \mathrm{Cs}$ & ${ }^{137} \mathrm{Cs}$ & ${ }^{226} \mathrm{Ra}$ & ${ }^{226} \mathrm{Ra}$ & ${ }^{238} \mathrm{P} \mathrm{u}$ & ${ }^{238} \mathrm{Pu}$ & ${ }^{239} \mathrm{Pu}+{ }^{240} \mathrm{Pu}$ & ${ }^{239} \mathrm{Pu}+{ }^{240} \mathrm{Pu}$ \\
\hline Unit & & $(f t)$ & $(0 \mathrm{Ci} / \mathrm{L})$ & $(p C i / L)$ & $(p \mathrm{Ci} / \mathrm{L})$ & $(p \mathrm{Ci} / L)$ & $(p C i / L)$ & $(p C i / L)$ & $(p \mathrm{Ci} / L)$ & $(p \mathrm{Ci} / \mathrm{L})$ & $(p C i / L)$ & $(p C i / L)$ & $(\rho C i / L)$ \\
\hline Half-life (a) & & & 1.0 & 2.7 & 2.7 & 30.2 & 30.2 & 1600 & 1600 & 87.7 & 87.7 & $24110 / 6537$ & $24110 / 6537$ \\
\hline Ref. date & & & $1 / 1 / 94$ & collect. & $1 / 1 / 94$ & collect. & $1 / 1 / 94$ & collect. & $1 / 1 / 94$ & collect. & $1 / 1 / 94$ & collect. & $1 / 1 / 94$ \\
\hline U.4u PS2a & Dalhart & 1680 & $4.31 e+2$ & $1.36 e+2$ & $3.51 \mathrm{e}+2$ & $6.65 e+1$ & $7.25 e+1$ & $2.81 e+0$ & $2.81 e+0$ & $2.61 \mathrm{e}-2$ & $2.68 \mathrm{e}-2$ & $1.92 \mathrm{e}-1$ & $1.92 e-1$ \\
\hline U-20n PS\#1DD-h & Cheshure & 2250 & $(4.98 e+1)$ & $(1.18 e+0)$ & $(3.01 e+0)$ & $2.19 e+1$ & $2.39 e+1$ & $3.13 e+0$ & $3.14 e+0$ & - & - & . &. \\
\hline U-20n PS\#1DD-h & Cheshire & 2550 & $(2.73 e+1)$ & $2.13 e+0$ & $5.45 e+0$ & $2.31 e+1$ & $2.52 e+1$ & $(8.01 e-1)$ & $(8.02 e-1)$ &. & - & . &. \\
\hline U-20n PS\#1DD-h & Cheshire & 2650 & $(4.92 \theta+1)$ & $1.04 e+1$ & $2.680+1$ & $2.35 e+1$ & $2.57 e+1$ & $2.220+0$ & $2.220+0$ & 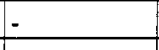 & - & - & F \\
\hline U.20n PS\#1DD-h & Cheshire & 2750 & $(2.94 e+1)$ & $3.30 e+0$ & $8.54 e+0$ & $4.65 e+0$ & $5.07 e+0$ & $6.99 e+0$ & $7.00 e+0$ & $(6.09 e-3)$ & $(6.26 e-3)$ & $(3.85 \mathrm{e}-3)$ & $(3.85 e-3)$ \\
\hline \multicolumn{14}{|c|}{ NOTE: ()$=$ detection limit. } \\
\hline$=$ analysis not $\mathrm{c}$ & npleted & s sé & & & & & & & & & & & \\
\hline
\end{tabular}


TABLE I (continued)

Cavity and Near-Cavity "Hot" Well Analyses

1997

\begin{tabular}{|c|c|c|c|c|c|c|c|c|c|c|c|c|c|c|c|}
\hline Well name & Event & Depth & $\begin{array}{l}\text { Collection } \\
\text { date }\end{array}$ & $\mathrm{pH}$ & $E C$ & $T$ & TDIC & $F$ & $\mathrm{Cl}$ & $\mathrm{SO}_{4}$ & $\mathrm{NO}_{3}$ & $\mathrm{Ca}$ & $\mathrm{Mg}$ & $\mathrm{Na}$ & $\mathrm{K}$ \\
\hline Unit & & $(f t)$ & & & $(\mu \mathrm{S} / \mathrm{cm})$ & $c$ & $(m g / L)$ & $(\mathrm{mg} / \mathrm{L})$ & $(m g / L)$ & $(\mathrm{mg} / \mathrm{L})$ & $(m g / L)$ & $(m g / L)$ & $(m g / L)$ & $(m g / L)$ & $(m g / L)$ \\
\hline U-4u PS2a & Dalhart & 1680 & $21-\mathrm{Ju} \mid-97$ & 7.3 & 332 & 28 & 157 & 0.47 & 5.92 & 9.5 & 19.3 & 6.53 & 1.45 & 61.3 & 11.2 \\
\hline U-20n PS\#1DD-h & Cheshire & 2250 & 22-Jul-97 & 7.8 & 353 & 28 & 63 & - & - & - &. & - & - & - & - \\
\hline U-20n PS\#1DD-h & Cheshıre & 2550 & $22-J u l-97$ & 8.8 & 347 & 29 & 78 & - & - & . & $=$ & - & - & - & - \\
\hline U-20n PS\#1DD-h & Cheshire & 2650 & 22-Jul-97 & 7.8 & 331 & 28 & 91 & - & . & - & - & - & $=$ & - & - \\
\hline U-20n PS\#1DD-h & Cheshire & 2750 & $23-J u l-97$ & 7 & - & - & 103 & 2.19 & 11.9 & 26.5 & 1.28 & 16.7 & 0.45 & 57.3 & 7.5 \\
\hline \multicolumn{16}{|c|}{ NOTE: ()$=$ detection limit..$\quad$ L } \\
\hline \multirow{2}{*}{\multicolumn{16}{|c|}{ * = analysis not completed on this sample. }} \\
\hline & & & $\mathrm{HCO}_{3}{ }^{\circ} \mathrm{L}$ & & & & & & & & & & & & \\
\hline
\end{tabular}

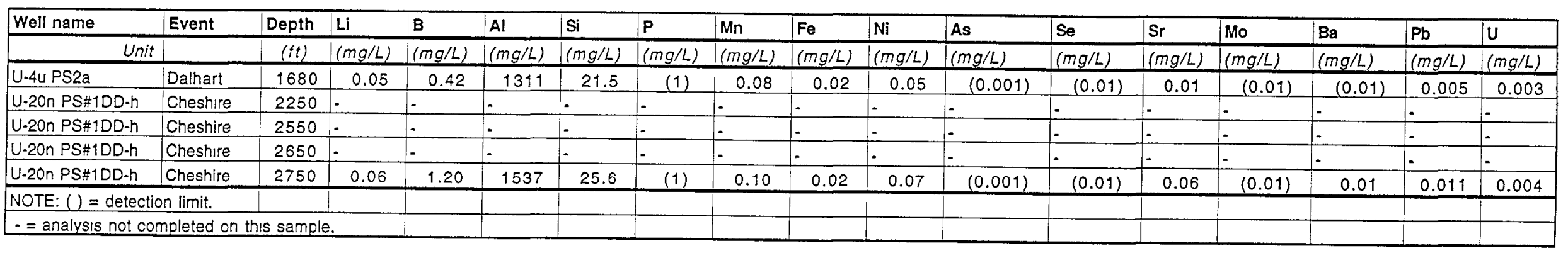




\section{CHAPTER 4}

\section{OLDER CAVITY OR NEAR-CAVITY "HOT" WELL DATA: FY77 - FY96}

Gail F. Eaton

The following is a compilation of data collected by LLNL in FY93, FY94, FY96 as well as representative historical data from the 1970s and 1980s. These tables are updated from tables that appeared in the LLNL HRMP FY 1996 Progress Report (Smith et al., 1997). The tables in HRMP FY 1995 Progress Report contain errors and should not be used. Refer to Figure 1 in Chapter 3 of this report for location of the cited underground nuclear tests.

The historical analyses are not a comprehensive list of all cavity fluids ever analyzed but rather analyses that LLNL has been able to verify through archived field and analytical records.

Table I includes data returned during FY96; Table II includes data returned in FY94; Table III includes data returned in FY93, and Table IV is a compilation of the older historical data.

Some wells were slant drilled and completed at an inclined angle. In all cases collection depths for the bailed samples represent measured distance from the surface down the well casing and not vertical depth below surface.

\section{REFERENCE}

Smith, D.K., A.B. Kersting, J.M. Kenneally, J.H. Rego, and J. L. Thompson, 1997, Hydrologic Resources Management Program FY 1996 Progress Report, Lawrence Livermore National Laboratory, UCRL-ID-126886, 40p. 
TABLE I

Cavity or Near-Cavity "Hot" Well Analyses

1996

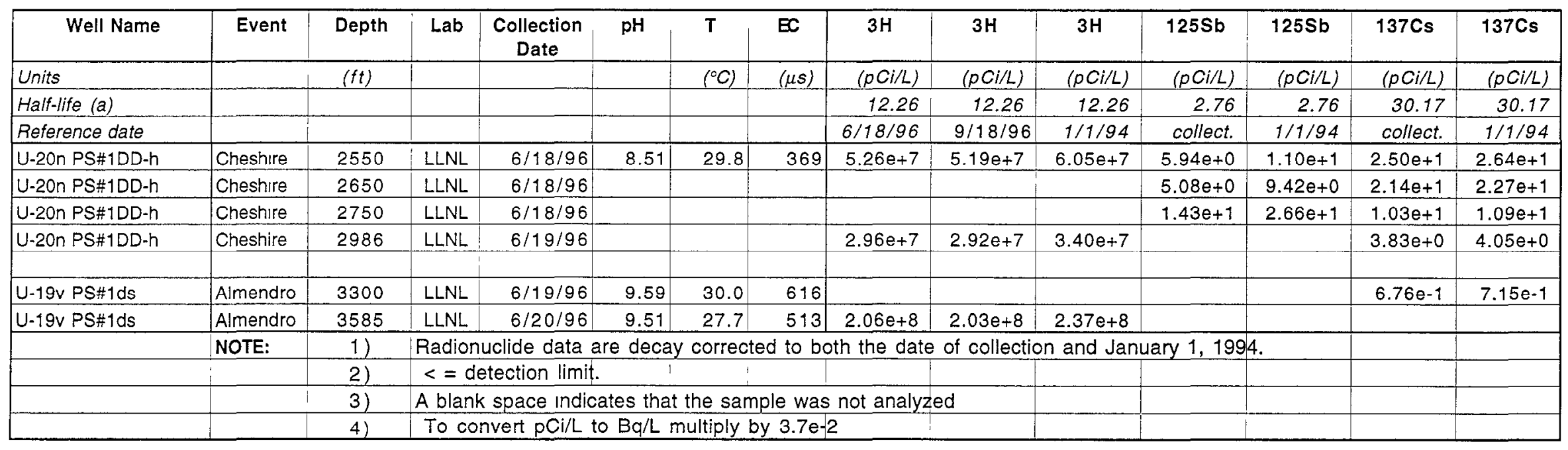


TABLE II

Cavity or Near-Cavity "Hot" Well Analyses

\section{4}

\begin{tabular}{|c|c|c|c|c|c|c|c|c|c|c|c|c|c|c|}
\hline Well Name & Event & Depth & Lab & $\begin{array}{l}\text { Collection } \\
\text { Date }\end{array}$ & DO & $\mathrm{pH}$ & $\mathbf{T}$ & $E C$ & $\begin{array}{l}\text { Gross } \\
\text { alpha }\end{array}$ & $\begin{array}{l}\text { Gross } \\
\text { beta }\end{array}$ & $3 \mathrm{H}$ & $3 \mathrm{H}$ & $14 C$ & $14 C$ \\
\hline Half-life (a) & & & & & & & & & & & 12.26 & 12.26 & 5730 & 5730 \\
\hline Reference date & & & & & & & & & collect. & collect. & collect. & $1 / 1 / 94$ & collect. & $1 / 1 / 94$ \\
\hline & & & & & & & & & & & & & & \\
\hline U-2gg PSE3A & Ingot & 1927 & LLNL & 22-Sep-94 & & & & & & & $6.49 \mathrm{e}+3$ & $6.76 e+3$ & $1.99 e+4$ & $1.99 e+4$ \\
\hline U.2gg PSE3A & Ingot & 1960 & LLNL & $21-$ Sep-94 & 1.5 & 7.47 & 26.0 & 2280 & & & $5.45 e+3$ & $5.68 e+3$ & $5.96 e+3$ & $5.96 e+3$ \\
\hline U-20n PS1DD-h & Cheshire & 2248 & LLNL & 14-Sep-94 & 2.0 & 8.35 & 27.8 & 386 & $<5.18 e+0$ & $8.85 e+1$ & $6.55 \mathrm{e}+7$ & $6.81 e+7$ & $5.29 e+4$ & $5.29 e+4$ \\
\hline$U-20 n$ PS1DD-h & Cheshire & 2448 & LLNL & 14-Sep-94 & & & & & & & $6.49 e+7$ & $6.75 e+7$ & & \\
\hline U-20n PS1DD-h & Cheshire & 2648 & LLNL & 14-Sep-94 & & & & & $<4.90 e+0$ & $7.71 \mathrm{e}+1$ & $6.34 e+7$ & $6.60 e+7$ & $6.12 e+4$ & $6.12 e+4$ \\
\hline & NOTE: & 1) & \multicolumn{10}{|c|}{ Radionuclide data are decay corrected to both the date of collection and January 1,1994 . } & & \\
\hline & & 2) & $<=d$ & detection limit. & & & & & & & & & & \\
\hline & & 3) & \multicolumn{7}{|c|}{ A blank space indicates that the sample was not analyzed } & & & & & \\
\hline & & 4) & \multicolumn{6}{|c|}{ To convert $\mathrm{pCi} / \mathrm{L}$ to $\mathrm{Bq} / \mathrm{L}$ multiply by $3.7 \mathrm{e}-2$} & & & & & & \\
\hline
\end{tabular}




\section{TABLE II}

Cavity or Near-Cavity "Hot" Well Analyses

1994

\begin{tabular}{|c|c|c|c|c|c|c|c|c|c|c|c|}
\hline Well Name & Event & $85 \mathrm{Kr}$ & $85 \mathrm{Kr}$ & $125 \mathrm{Sb}$ & $125 \mathrm{Sb}$ & $137 \mathrm{Cs}$ & $137 \mathrm{Cs}$ & 238Pu & 238Pu & $239,240 \mathrm{Pu}$ & $239,240 \mathrm{Pu}$ \\
\hline Units & & $(\rho C i / L)$ & $(p C i / L)$ & $(p C i / L)$ & $(p C i / L)$ & $(p C i / L)$ & $(p C i / L)$ & $(p C i / L)$ & $(p C i / L)$ & $(p C i / L)$ & $(p C i / L)$ \\
\hline Half-life (a) & & 10.72 & 10.72 & 2.76 & 2.76 & 30.17 & 30.17 & 87.74 & 87.74 & 6560 & 6560 \\
\hline Reference date & & collect. & $1 / 1 / 94$ & collect. & $1 / 1 / 94$ & collect. & $1 / 1 / 94$ & collect. & $1 / 1 / 94$ & collect. & $1 / 1 / 94$ \\
\hline Sample Blank & & & & $<5.97 \mathrm{e}-1$ & $<7.11 \mathrm{e}-1$ & $<2.50 \mathrm{e}-1$ & $<2.54 \mathrm{e}-1$ & $<4.33 \mathrm{e}-3$ & $<4.35 \mathrm{e}-3$ & $<2.67 \mathrm{e}-3$ & $<2.67 \mathrm{e}-3$ \\
\hline U-2gg PSE $3 A$ & Ingot & & & $<9.59 \mathrm{e}-1$ & $<1.15 e+0$ & $8.98 e-1$ & $913 e-1$ & $<3.71 \mathrm{e}-3$ & $<3.73 e-3$ & $<3.05 e-3$ & $<3.05 e-3$ \\
\hline U-2gg PSE3A & Ingot & & & $<1.23 e+0$ & $<1.47 e+0$ & $2.74 e+0$ & $2.79 e+0$ & $<4.51 \mathrm{e}-3$ & $<4.54 \mathrm{e}-3$ & $<2.74 \mathrm{e}-3$ & $<2.74 \mathrm{e}-3$ \\
\hline U-2gg PSE3A & Ingot & $1.00 e+1$ & $1.05 e+1$ & & & & & & & & \\
\hline & & & & & & & & & & & \\
\hline U-20n PS1DD-h & Cheshire & & & $<1.01 e+0$ & $<1.20 e+0$ & $2.33 e+1$ & $2.37 e+1$ & $1.88 \mathrm{e}-2$ & $1.89 \mathrm{e}-2$ & $8.53 e-2$ & $8.53 \mathrm{e}-2$ \\
\hline U-20n PS1DD-h & Cheshire & & & $<9.47 e-1$ & $<1.13 e+0$ & $2.34 e+1$ & $2.38 e+1$ & $<3.26 \mathrm{e}-3$ & $<3.28 \mathrm{e}-3$ & $<3.63 e-3$ & $<3.63 e-3$ \\
\hline U-20n PS1DD-h & Cheshire & & & $3.60 \mathrm{e}+0$ & $4.29 e+0$ & $1.83 e+1$ & $1.86 e+1$ & $<5.16 \mathrm{e}-3$ & $<5.19 e-3$ & $1.00 e-2$ & $1.00 \mathrm{e}-2$ \\
\hline
\end{tabular}


TABLE III

Cavity or Near-Cavity "Hot" Well Analyses

1993

\begin{tabular}{|c|c|c|c|c|c|c|c|c|c|c|c|c|c|}
\hline Well name & Event & Depth & Lab & $\begin{array}{l}\text { Collection } \\
\text { date }\end{array}$ & HNO3/filter & DO & $\mathrm{pH}$ & $E C$ & TDS & Alkalinity & $\mathrm{Li}$ & $B$ & $\mathrm{Na}$ \\
\hline Unit & & $(f t)$ & & & & $(m g / L)$ & & $(\mu S)$ & $(\mu g / g)$ & $(\mathrm{mg} \mathrm{CaCO} / \mathrm{L})$ & $(m g / L)$ & $(m g / L)$ & $(m g / L)$ \\
\hline \multicolumn{14}{|l|}{ Half-life (a) } \\
\hline \multicolumn{14}{|l|}{ Ref. date } \\
\hline RNM-1 & Cambric & pump & LLNL & 14-Sep-93 & no/no & & & & & & $<0.1$ & 0.24 & 43 \\
\hline RNM-2s & Cambric & pump & LLNL & 14-Sep-93 & no/no & & & & & & $<0.1$ & 0.24 & 49 \\
\hline \multicolumn{14}{|c|}{1} \\
\hline U-19v-PS1ds & Almendro & 2464 & LLNL & 27-Sep-93 & yes $/ 0.2 \mathrm{~mm}$ & 1.5 & 8.8 & 7.93 & 394 & 20 & 0.4 & 1.02 & 152 \\
\hline U-19v-PS1ds & Almendro & 3300 & LLNL & 29-Sep-93 & yes $/ 0.2 \mathrm{~mm}$ & 2.0 & 9.1 & 759 & 380 & 50 & 0.2 & 1.32 & 155 \\
\hline U-4t-PS3a & Gascon & 1055 & LLNL & 31-Aug-93 & yes/no & 3.0 & 7.6 & 787 & 377 & $<200$ & 0.2 & 0.34 & 86 \\
\hline U-4u-PS2a & Dalhart & 1750 & LLNL & 31-Aug-93 & yes/no & 3.0 & 7.6 & 420 & 209 & 350 & $<0.1$ & 0.13 & 105 \\
\hline \multirow{2}{*}{\multicolumn{14}{|c|}{ UE-2ce }} \\
\hline & & & & & & & & & & & & & \\
\hline UE-3e\#4 (p3) & Aleman & 1630 & LLNL & 25-Aug-93 & yes $/ 0.2 \mathrm{~mm}$ & & 12.5 & 8,040 & 4,030 & $>1200$ & $<0.1$ & 0.35 & 32 \\
\hline UE-3e\#4 (p2) & Aleman & 1785 & LLNL & 26-Aug-93 & yes $/ 0.2 \mathrm{~mm}$ & 3.5 & 11.5 & 3,800 & 2,070 & & 0.2 & $<0.06$ & 239 \\
\hline UE-3e\#4 (p1) & Aleman & 1885 & LLNL & 26-Aug-93 & yes $/ 0.2 \mathrm{~mm}$ & & & & & & & & \\
\hline UE-3e\#4 (p1) & Aleman & 2150 & LLNL & 25-Aug-93 & yes $/ 0.2 \mathrm{~mm}$ & 3.0 & 11.3 & 2,290 & 1,150 & 320 & $<0.1$ & 0.20 & 220 \\
\hline \multirow[t]{5}{*}{ UE-7ns } & Bourbon & 2025 & LLNL & 24-Aug-93 & yes/no & 2.5 & & 434 & 223 & 170 & $<0.1$ & 0.16 & 59 \\
\hline & NOTE: & 1) & \multicolumn{9}{|c|}{ Radionuclide data are decay corrected to both the date of collection and January 1,1994 . } & & \\
\hline & & 2) & \multicolumn{3}{|c|}{$<=$ detection limit. } & & & & & & & & \\
\hline & & 3) & \multicolumn{6}{|c|}{ A blank space indicates that the sample was not analyzed } & & & & & \\
\hline & & 4) & \multicolumn{5}{|c|}{ To convert $\mathrm{pCi} / \mathrm{L}$ to $\mathrm{Bg} / \mathrm{L}$ multiply by $3.7 \mathrm{e}-2$} & & & & & & \\
\hline
\end{tabular}


TABLE III

Cavity or Near-Cavity "Hot" Well Analyses

1993

\begin{tabular}{|c|c|c|c|c|c|c|c|c|c|c|c|c|c|}
\hline Well name & Event & $\mathrm{Mg}$ & Al & $\mathrm{Si}$ & $P$ & $s$ & $\mathrm{Cl}$ & $\mathrm{K}$ & $\mathrm{Ca}$ & $\mathrm{Or}$ & $M n$ & $\mathrm{Fe}$ & Co \\
\hline Unit & & $(m g / L)$ & $(m g / L)$ & $(\mathrm{mg} / \mathrm{L})$ & $(m g / L)$ & $(m g / L)$ & $(m g / L)$ & $(m g / L)$ & $(m g / L)$ & $(n g / g)$ & $(n g / g)$ & $(m g / L)$ & $(n g / g)$ \\
\hline \multicolumn{14}{|l|}{ Half-life (a) } \\
\hline \multicolumn{14}{|l|}{ Ref. date } \\
\hline RNM-1 & Cambric & 9.97 & $<0.06$ & 30.5 & 0.1 & 14 & & 8.0 & 26.1 & 1.4 & 0.4 & 0.03 & $<0.1$ \\
\hline RNM-2s & Cambric & 8.13 & $<0.06$ & 26.6 & $<0.1$ & 12 & & 8.9 & 20.9 & 1.0 & 22.5 & 0.06 & $<0.1$ \\
\hline U-19v-PS1ds & Almendro & 0.21 & $<0.06$ & 3.9 & 0.3 & 1 & 90.0 & 22.9 & 2.3 & 1.5 & 2.8 & 0.14 & $<0.1$ \\
\hline U-19v-PS1ds & Almendro & 0.03 & $<0.06$ & 10.5 & $<0.1$ & 2 & 171.6 & 11.7 & 1.2 & 0.6 & 2.0 & 0.12 & $<0.1$ \\
\hline U-4t-PS3a & Gascon & 2.19 & 0.09 & 1.4 & $<0.1$ & 21 & 31.7 & 9.6 & 5.7 & 2.4 & 87.8 & 4.01 & 0.4 \\
\hline U-4u-PS2a & Dalhart & 2.96 & 0.15 & 25.7 & $<0.1$ & 5 & 9.1 & 24.7 & 32.9 & 2.0 & 132.5 & 1.58 & 0.3 \\
\hline UE-2ce & Nash & 25.32 & 1.54 & 22.9 & $<0.1$ & 5 & 18.3 & 19.5 & 57.7 & 1.6 & 31.6 & 0.90 & $<0.1$ \\
\hline & & & & & & & & & & & & & \\
\hline UE-3e\#4 (p3) & Aleman & $<0.01$ & 0.20 & 2.6 & $<0.1$ & 8 & 496.7 & 10.8 & 893.0 & 7.1 & 0.7 & $<0.02$ & 10.8 \\
\hline UE-3e\#4 (p2) & Aleman & $<0.01$ & 14.42 & 2.4 & $<0.1$ & 4 & 188.2 & 48.5 & 217.0 & 91.2 & $<0.2$ & $<0.02$ & 1.0 \\
\hline UE-3e\#4 (p1) & Aleman & & & & & & & & & & & & \\
\hline UE-3e\#4 (p1) & Aleman & 0.01 & 0.20 & 6.4 & $<0.1$ & 10 & 67.5 & 10.3 & 76.9 & 46.2 & $<0.2$ & $<0.02$ & 0.4 \\
\hline UE-7ns & Bourbon & 4.19 & 2.22 & 9.6 & $<0.1$ & 1 & 37.0 & 4.9 & 21.2 & 2.3 & 55.6 & 0.28 & $<0.1$ \\
\hline
\end{tabular}


TABLE III

Cavity or Near-Cavity "Hot" Well Analyses

1993

\begin{tabular}{|c|c|c|c|c|c|c|c|c|c|c|c|c|c|c|}
\hline Well name & Event & $\overline{\mathrm{Ni}}$ & $\mathrm{Cu}$ & $\mathrm{Zn}$ & As & $\mathrm{Se}$ & $\mathrm{Sr}$ & Mo & $\mathrm{Ba}$ & $\mathrm{Hg}$ & $\mathrm{Pb}$ & $\mathrm{U}$ & $\begin{array}{l}\text { Gross } \\
\text { alpha }\end{array}$ & $\begin{array}{l}\text { Gross } \\
\text { beta }\end{array}$ \\
\hline Unit & & $(n g / g)$ & $(n g / g)$ & $(n g / g)$ & $(n g / g)$ & $(n g / g)$ & $(n g / g)$ & $(n g / g)$ & $(n g / g)$ & $(n g / g)$ & $(n g / g)$ & $(n g / g)$ & $(\mathrm{pCi} / \mathrm{L})$ & $(p C i / L)$ \\
\hline \multicolumn{15}{|l|}{ Half-life (a) } \\
\hline Ref. date & & & & & & & & & & & & & collect. & collect. \\
\hline RNM-1 & Cambric & $<0.1$ & 1.1 & 8.0 & 7.0 & 8.4 & 266 & 4 & 15 & 1.1 & 0.8 & 3.9 & $<3.4 e+1$ & $<7.0 e+1$ \\
\hline RNM-2s & Cambric & $<0.1$ & 1.0 & 2.0 & 5.0 & 9.5 & 120 & 4 & 24 & 1.0 & 1.4 & 3.5 & $<3.4 e+1$ & $<7.0 e+1$ \\
\hline & & & & & & & & & & & & & & \\
\hline U-19v-PS1ds & Almendro & $<0.1$ & 3.2 & 59.5 & 31.0 & 36.9 & 269 & 687 & 100 & $<0.5$ & 1.5 & $<0.1$ & $<3.4 \mathrm{e}+1$ & $<7.0 \mathrm{e}+1$ \\
\hline U-19v-PS1ds & Almendro & $<0.1$ & 1.5 & 24.0 & 105.0 & 23.0 & 47 & 1,007 & 49 & 2.0 & 0.8 & $<0.1$ & $<3.4 e+1$ & $<7.0 e+1$ \\
\hline U-4t-PS3a & Gascon & 24.0 & 31.2 & $3,323.3$ & 5.8 & 28.5 & 963 & 111 & 127 & $<0.5$ & 194.0 & 0.3 & $<3.4 e+1$ & $<7.0 e+1$ \\
\hline U-4u-PS2a & Dalhart & 26.7 & 63.1 & 409.7 & 9.5 & 16.9 & 46 & 2 & 9 & 1.0 & 4.4 & 6.3 & $<3.4 e+1$ & $7.3 e+2$ \\
\hline UE-2ce & Nash & $<0.1$ & 3.2 & 314.7 & 1.6 & 5.5 & 217 & 14 & 30 & $<0.5$ & 23.5 & 0.4 & $<3.4 e+1$ & $<7.0 e+1$ \\
\hline & & & & & & & & & & & & & & \\
\hline UE-3e\#4 (p3) & Aleman & 71.0 & 190.3 & 585.4 & 194.2 & 64.6 & 8,084 & 29 & 1,140 & 9.1 & 855.9 & $<0.1$ & $<3.4 e+1$ & $<7.0 e+1$ \\
\hline UE-3e\#4 (p2) & Aleman & 1.1 & 40.4 & 117.9 & 14.9 & 73.4 & 4,471 & 26 & 1,518 & 3.1 & 8.5 & $<0.1$ & $<3.4 e+1$ & $<7.0 \mathrm{e}+1$ \\
\hline UE-3e\#4 (p1) & Aleman & & & & & & & & & & & & $<3.4 \mathrm{e}+1$ & $<7.0 e+1$ \\
\hline UE-3e\#4 (p1) & Aleman & $<0.1$ & 60.3 & 6.4 & 7.2 & 30.7 & 487 & 42 & 144 & 1.1 & 18.0 & $<0.1$ & $<3.4 e+1$ & $<7.0 e+1$ \\
\hline UE-7ns & Bourbon & $<0.1$ & 8.8 & 234.0 & 4.8 & 8.1 & 144 & 36 & 125 & $<0.5$ & 15.0 & $<0.1$ & $<3.4 \theta+1$ & $<7.0 e+1$ \\
\hline
\end{tabular}


TABLE III

\section{Cavity or Near-Cavity "Hot" Well Analyses \\ 1993}

\begin{tabular}{|c|c|c|c|c|c|c|c|c|c|c|c|}
\hline Well name & Event & $3 \mathrm{H}$ & $3 \mathrm{H}$ & $14 \mathrm{C}$ & $14 \mathrm{C}$ & $60 \mathrm{Co}$ & $60 \mathrm{Co}$ & $125 \mathrm{Sb}$ & $125 \mathrm{Sb}$ & $137 \mathrm{Cs}$ & $137 \mathrm{Cs}$ \\
\hline Unit & & $(p C i / L)$ & $(p C i / L)$ & $(p C i / L)$ & $(p C i / L)$ & $(p C i / L)$ & $(p C i / L)$ & $(p C i / L)$ & $(p C i / L)$ & $(p C i / L)$ & $(p C i / L)$ \\
\hline Half-life (a) & & 12.26 & 12.26 & 5730 & 5730 & 5.27 & 5.27 & 2.76 & 2.76 & 30.17 & 30.17 \\
\hline Ref. date & & collect. & $1 / 1 / 94$ & collect. & $1 / 1 / 94$ & collect. & $1 / 1 / 94$ & collect. & $1 / 1 / 94$ & collect. & $1 / 1 / 94$ \\
\hline RNM-1 & Cambric & $<1.00 e+3$ & $<9.83 e+2$ & $<3.20 e+3$ & $<3.20 e+3$ & $<3.30 \mathrm{e}-1$ & $<3.17 e-1$ & $<9.74 e-1$ & $<9.04 \mathrm{e}-1$ & $6.85 e+0$ & $6.80 e+0$ \\
\hline RNM-2s & Cambric & $2.60 e+5$ & $2.56 e+5$ & $<3.20 e+3$ & $<3.20 e+3$ & $<2.25 e-1$ & $<2.16 e-1$ & $<6.84 e-1$ & $<6.35 e-1$ & $<2.41 e-1$ & $<2.39 e-1$ \\
\hline U-19v-PS1ds & Almendro & $2.30 e+8$ & $2.27 e+8$ & $<3.20 e+3$ & $<3.20 e+3$ & $<3.24 \mathrm{e}-1$ & $<3.13 e-1$ & $<8.43 e-1$ & $<7.89 e-1$ & $<3.87 e-1$ & $<3.85 \mathrm{e}-1$ \\
\hline U-19v-PS1ds & Almendro & $2.30 e+8$ & $2.27 e+8$ & $<3.20 \mathrm{e}+3$ & $<3.20 e+3$ & $<2.26 \mathrm{e}-1$ & $<2.18 \mathrm{e}-1$ & $<5.50 e-1$ & $<5.16 \mathrm{e}-1$ & $<2.49 \mathrm{e}-1$ & $<2.48 \mathrm{e}-1$ \\
\hline U-4t-PS3a & Gascon & $6.70 e+4$ & $6.57 e+4$ & $<3.20 \mathrm{e}+3$ & $<3.20 \mathrm{e}+3$ & $<3.34 \mathrm{e}-1$ & $<3.20 \mathrm{e}-1$ & $<8.75 e-1$ & $<8.04 \mathrm{e}-1$ & $<3.41 \mathrm{e}-1$ & $<3.38 \mathrm{e}-1$ \\
\hline U-4u-PS2a & Dalhart & $4.92 e+7$ & $4.83 e+7$ & $<3.20 \mathrm{e}+3$ & $<3.20 \mathrm{e}+3$ & $1.02 e+0$ & $9.76 e-1$ & $1.53 e+2$ & $1.40 e+2$ & $2.02 e+0$ & $2.00 e+0$ \\
\hline UE-2ce & Nash & $1.27 e+5$ & $1.24 e+5$ & $<3.20 \mathrm{e}+3$ & $<3.20 \mathrm{e}+3$ & $<3.04 e-1$ & $<2.90 \mathrm{e}-1$ & $<8.56 \mathrm{e}-1$ & $<7.83 \mathrm{e}-1$ & $<2.83 \mathrm{e}-1$ & $<2.81 e-1$ \\
\hline & & & & & & & & & & & \\
\hline UE-3e\#4 (p3) & Aleman & $4.39 e+5$ & $4.30 e+5$ & $4.77 e+3$ & $4.77 e+3$ & $<4.42 \mathrm{e}-1$ & $<4.22 e-1$ & $<1.26 e+0$ & $<1.15 e+0$ & $<4.72 \mathrm{e}-1$ & $<4.68 \mathrm{e}-1$ \\
\hline UE-3e\#4 (p2) & Aleman & $4.77 e+4$ & $4.68 e+4$ & $<3.20 e+3$ & $<3.20 e+3$ & $<2.72 e-1$ & $<2.60 \mathrm{e}-1$ & $<7.66 \mathrm{e}-1$ & $<7.01 e-1$ & $<2.85 \mathrm{e}-1$ & $<2.83 e-1$ \\
\hline UE-3e\#4 (p1) & Aleman & $3.30 \mathrm{e}+5$ & $3.24 e+5$ & & & & & & & & \\
\hline UE-3e\#4 (p1) & Aleman & $9.42 e+6$ & $9.23 e+6$ & $<3.20 e+3$ & $<3.20 e+3$ & & & & & & \\
\hline & & & & & & & & & & & \\
\hline UE-7ns & Bourbon & $4.57 e+2$ & $4.48 e+2$ & $<3.20 e+3$ & $<3.20 e+3$ & $<2.43 e-1$ & $<2.32 \mathrm{e}-1$ & $<5.84 e-1$ & $<5.34 e-1$ & $<2.50 e-1$ & $<2.48 \mathrm{e}-1$ \\
\hline
\end{tabular}




\section{TABLE III}

Cavity or Near-Cavity "Hot" Well Analyses

1993

\begin{tabular}{|c|c|c|c|c|c|c|c|c|c|c|c|}
\hline Well name & Event & $3 \mathrm{H}$ & $3 \mathrm{H}$ & $14 C$ & $14 C$ & $60 \mathrm{Co}$ & $60 \mathrm{Co}$ & $125 \mathrm{Sb}$ & $125 \mathrm{Sb}$ & $137 \mathrm{Cs}$ & $137 \mathrm{Cs}$ \\
\hline Unit & & $(p C I / L)$ & $(p C i / L)$ & $(p C I / L)$ & $(p C i / L)$ & $(p C i / L)$ & $(p C i / L)$ & $(p C i / L)$ & $(p C i / L)$ & $(p \subset i / L)$ & $(p C i / L)$ \\
\hline Half-life (a) & & 12.26 & 12.26 & 5730 & 5730 & 5.27 & 5.27 & 2.76 & 2.76 & 30.17 & 30.17 \\
\hline Ref. date & & collect. & $1 / 1 / 94$ & collect. & $1 / 1 / 94$ & collect. & $1 / 1 / 94$ & collect. & $1 / 1 / 94$ & collect. & $1 / 1 / 94$ \\
\hline RNM-1 & Cambric & $<1.00 e+3$ & $<9.83 e+2$ & $<3.20 e+3$ & $<3.20 e+3$ & $<3.30 \mathrm{e}-1$ & $<3.17 \mathrm{e}-1$ & $<9.74 e-1$ & $<9.04 e-1$ & $6.85 e+0$ & $6.80 e+0$ \\
\hline RNM-2s & Cambric & $2.60 e+5$ & $2.56 e+5$ & $<3.20 e+3$ & $<3.20 e+3$ & $<2.25 e-1$ & $<2.16 \mathrm{e}-1$ & $<6.84 e-1$ & $<6.35 e-1$ & $<2.41 e-1$ & $<2.39 e-1$ \\
\hline U-19v-PS1ds & Almendro & $2.30 \mathrm{e}+8$ & $2.27 e+8$ & $<3.20 e+3$ & $<3.20 \mathrm{e}+3$ & $<3.24 \mathrm{e}-1$ & $<3.13 e-1$ & $<8.43 e-1$ & $<7.89 \mathrm{e}-1$ & $<3.87 e-1$ & $<3.85 \mathrm{e}-1$ \\
\hline U-19v-PS1ds & Almendro & $2.30 e+8$ & $2.27 \theta+8$ & $<3.20 \theta+3$ & $<3.20 e+3$ & $<2.26 \theta-1$ & $<2.18 e-1$ & $<5.50 \mathrm{e}-1$ & $<5.16 e-1$ & $<2.49 e-1$ & $<2.48 e-1$ \\
\hline U-4t-PS3a & Gascon & $6.70 e+4$ & $6.57 e+4$ & $<3.20 \mathrm{e}+3$ & $<3.20 e+3$ & $<3.34 e-1$ & $<3.20 \mathrm{e}-1$ & $<8.75 \mathrm{e}-1$ & $<8.04 \mathrm{e}-1$ & $<3.41 \mathrm{e}-1$ & $<3.38 \mathrm{e}-1$ \\
\hline U-4u-PS2a & Dalhart & $4.92 e+7$ & $4.83 e+7$ & $<3.20 \mathrm{e}+3$ & $<3.20 \mathrm{e}+3$ & $1.02 e+0$ & $9.76 \mathrm{e}-1$ & $1.53 e+2$ & $1.40 \mathrm{e}+2$ & $2.02 e+0$ & $2.00 e+0$ \\
\hline UE-2ce & Nash & $1.27 e+5$ & $1.24 e+5$ & $<3.20 e+3$ & $<3.20 e+3$ & $<3.04 e-1$ & $<2.90 e-1$ & $<8.56 e-1$ & $<7.83 e-1$ & $<2.83 e-1$ & $<2.81 e-1$ \\
\hline UE-3e\#4 (p3) & Aleman & $4.39 e+5$ & $4.30 e+5$ & $4.77 e+3$ & $4.77 e+3$ & $<4.42 \mathrm{e}-1$ & $<4.22 \mathrm{e}-1$ & $<1.26 e+0$ & $<1.15 e+0$ & $<4.72 \mathrm{e}-1$ & $<4.68 \mathrm{e}-1$ \\
\hline UE-3e\#4 (p2) & Aleman & $4.77 e+4$ & $4.68 e+4$ & $<3.20 e+3$ & $<3.20 \mathrm{e}+3$ & $<2.72 e-1$ & $<2.60 e-1$ & $<7.66 \mathrm{e}-1$ & $<7.01 \mathrm{e}-1$ & $<2.85 \mathrm{e}-1$ & $<2.83 \mathrm{e}-1$ \\
\hline UE-3e\#4 (p1) & Aleman & $3.30 e+5$ & $3.24 e+5$ & & & & & & & & \\
\hline UE-3e\#4 (p1) & Aleman & $9.42 e+6$ & $9.23 e+6$ & $<3.20 \mathrm{e}+3$ & $<3.20 e+3$ & & & & & & \\
\hline UE-7ns & Bourbon & $4.57 e+2$ & $4.48 e+2$ & $<3.20 e+3$ & $<3.20 \mathrm{e}+3$ & $<2.43 \mathrm{e}-1$ & $<2.32 e-1$ & $<5.84 e-1$ & $<5.34 \mathrm{e}-1$ & $<2.50 \mathrm{e}-1$ & $<2.48 \mathrm{e}-1$ \\
\hline
\end{tabular}


TABLE III

\section{Cavity or Near-Cavity "Hot" Well Analyses \\ 1993}

\begin{tabular}{|c|c|c|c|c|c|c|c|c|c|c|c|}
\hline Well name & Event & 226Ra & 226Ra & $238 \mathrm{Pu}$ & $238 \mathrm{Pu}$ & $239,240 \mathrm{Pu}$ & $239,240 \mathrm{Pu}$ & \begin{tabular}{|l|} 
Carbon \\
disulfide
\end{tabular} & $\begin{array}{l}\text { TCE } \\
1,1,1 \\
\end{array}$ & Benzene & $\begin{array}{l}\text { Methylene } \\
\text { chloride }\end{array}$ \\
\hline Unit & & $(p C i / L)$ & $(p C i / L)$ & $(p C i / L)$ & $(p C i / L)$ & $(p C i / L)$ & $(p \subset i / L)$ & $(\mu g / L)$ & $(\mu g / L)$ & $(\mu g / L)$ & $(\mu g / L)$ \\
\hline Half-life (a) & & 1600 & 1600 & 87.74 & 87.74 & 6560 & 6560 & & & & \\
\hline Ref. date & & collect. & $1 / 1 / 94$ & collect. & $1 / 1 / 94$ & collect. & $1 / 1 / 94$ & & & & \\
\hline RNM-1 & Cambric & $<7.37 e-1$ & $<7.37 \mathrm{e}-1$ & $<6.35 e-2$ & $<6.34 e-2$ & $<5.35 e-2$ & $<5.35 e-2$ & 1.0 & 2.0 & $<0.3$ & $<0.3$ \\
\hline RNM-2s & Cambric & $1.71 e+0$ & $1.71 e+0$ & $<1.09 e-1$ & $<1.09 \mathrm{e}-1$ & $<6.97 e-2$ & $<6.97 e-2$ & 1.0 & 1.0 & 0.7 & 0.3 \\
\hline U-19v-PS1ds & Almendro & $<6.97 e-1$ & $<6.97 \mathrm{e}-1$ & $<2.29 \mathrm{e}-1$ & $<2.29 \mathrm{e}-1$ & $<1.36 \mathrm{e}-1$ & $<1.36 \mathrm{e}-1$ & 6.3 & 2.0 & 30.0 & 0.3 \\
\hline U-19v-PS1ds & Almendro & $<4.98 \mathrm{e}-1$ & $<4.98 \mathrm{e}-1$ & $<8.24 \mathrm{e}-1$ & $<8.22 e-1$ & $<6.64 \mathrm{e}-1$ & $<6.64 e-1$ & 0.4 & $<1.0$ & 4.0 & $<0.3$ \\
\hline U-4t-PS3a & Gascon & $<6.48 \mathrm{e}-1$ & $<6.48 \mathrm{e}-1$ & $<8.52 e-2$ & $<8.50 \mathrm{e}-2$ & $<4.21 \mathrm{e}-2$ & $<4.21 e-2$ & 2.9 & 1.0 & 1.0 & $<0.3$ \\
\hline U-4u-PS2a & Dalhart & $<5.55 e-1$ & $<5.55 \mathrm{e}-1$ & $<1.25 \mathrm{e}-1$ & $<1.25 \mathrm{e}-1$ & $1.36 e-1$ & $1.36 e-1$ & $<0.2$ & $<1.0$ & $<0.3$ & $<0.3$ \\
\hline & & & & & & & & & & & \\
\hline UE-2ce & Nash & $6.59 e+0$ & $6.59 e+0$ & $<1.02 e^{-1}$ & $<1.02 \mathrm{e}-1$ & $<8.56 \mathrm{e}-2$ & $<8.56 \mathrm{e}-2$ & 0.8 & 0.5 & 1.0 & $<0.3$ \\
\hline UE-3e\#4 (p3) & Aleman & $<9.56 e-1$ & $<9.56 e-1$ & $<7.83 e-2$ & $<7.81 e-2$ & $<7.52 \mathrm{e}-2$ & $<7.52 \mathrm{e}-2$ & $<0.2$ & $<1.0$ & $<0.3$ & 0.5 \\
\hline UE-3e\#4 (p2) & Aleman & $3.17 e+0$ & $3.17 e+0$ & $<1.29 \mathrm{e}-1$ & $<1.29 \mathrm{e}-1$ & $<7.75 \mathrm{e}-2$ & $<7.75 e-2$ & $<0.2$ & $<1.0$ & $<0.3$ & $<0.3$ \\
\hline UE-3e\#4 (p1) & Aleman & & & $<1.07 \mathrm{e}-1$ & $<1.07 e-1$ & $<6.40 \mathrm{e}-2$ & $<6.40 e-2$ & $<0.2$ & $<1.0$ & $<0.3$ & $<0.3$ \\
\hline UE-3e\#4 (p1) & Aleman & & & & & & & $<0.2$ & 1.0 & $<0.3$ & 0.6 \\
\hline & & & & & & & & & & & \\
\hline UE-7ns & Bourbon & $<5.00 e-1$ & $<5.00 e-1$ & $<6.85 e-2$ & $<6.83 e-2$ & $<7.36 e-2$ & $<7.36 e-2$ & $<0.2$ & $<1.0$ & $<0.3$ & $<0.3$ \\
\hline
\end{tabular}


TABLE III

Cavity or Near-Cavity "Hot" Well Analyses

1993

\begin{tabular}{|c|c|c|c|c|c|c|c|c|c|c|}
\hline Well name & Event & Toluene & \begin{tabular}{|l|}
$2-$ \\
Butanone \\
\end{tabular} & \begin{tabular}{|l|}
$\begin{array}{l}\text { Dimethyl } \\
\text { ether }\end{array}$ \\
\end{tabular} & $\begin{array}{l}\begin{array}{l}\text { 4-Methyl } \\
\text { phenol }\end{array} \\
\end{array}$ & \begin{tabular}{|l|}
$\begin{array}{l}\text { Cloro- } \\
\text { benzene }\end{array}$ \\
\end{tabular} & \begin{tabular}{|l|} 
Ethyl \\
Benzene
\end{tabular} & \begin{tabular}{|l} 
Vinal \\
Acetate
\end{tabular} & Acetone & $\begin{array}{l}\text { Chloro- } \\
\text { methane }\end{array}$ \\
\hline Unit & & $(\mu g / L)$ & $(\mu g / L)$ & $(\mu g / L)$ & $(\mu g / L)$ & $(\mu g / L)$ & $(\mu g / L)$ & $(\mu g / L)$ & $(\mu g / L)$ & $(\mu g / L)$ \\
\hline \multicolumn{11}{|l|}{ Half-life (a) } \\
\hline \multicolumn{11}{|l|}{ Ref. date } \\
\hline RNM-1 & Cambric & $<0.3$ & $<4.0$ & 20.0 & $<37.0$ & $<0.3$ & $<0.3$ & $<0.1$ & $<10.0$ & $<1.0$ \\
\hline RNM-2s & Cambric & 0.5 & $<4.0$ & 20.0 & $<37.0$ & $<0.3$ & $<0.3$ & $<0.1$ & $<10.0$ & $<1.0$ \\
\hline & & & & & & & & & & \\
\hline U-19v-PS1ds & Almendro & 20.0 & 4.0 & $<20.0$ & 65.0 & 18.0 & 0.7 & $<0.1$ & $<10.0$ & $<1.0$ \\
\hline U-19v-PS1ds & Almendro & $<0.3$ & 10.0 & $<20.0$ & $<37.0$ & $<0.3$ & $<0.3$ & $<0.1$ & 60.0 & $<1.0$ \\
\hline U-4t-PS3a & Gascon & 0.6 & $<4.0$ & 20.0 & $<37.0$ & $<0.3$ & $<0.3$ & $<0.1$ & $<10.0$ & $<1.0$ \\
\hline U-4u-PS2a & Dalhart & $<0.3$ & $<4.0$ & 30.0 & $<37.0$ & $<0.3$ & $<0.3$ & $<0.1$ & $<10.0$ & $<1.0$ \\
\hline UE-2ce & Nash & 0.6 & $<4.0$ & 20.0 & $<37.0$ & $<0.3$ & $<0.3$ & $<0.1$ & $<10.0$ & $<1.0$ \\
\hline & & & & & & & & & & \\
\hline UE-3e\#4 (p3) & Aleman & $<0.3$ & $<4.0$ & 30.0 & $<37.0$ & $<0.3$ & $<0.3$ & $<0.1$ & $<10.0$ & $<1.0$ \\
\hline UE-3e\#4 (p2) & Aleman & $<0.3$ & $<4.0$ & 20.0 & $<37.0$ & $<0.3$ & $<0.3$ & $<0.1$ & $<10.0$ & $<1.0$ \\
\hline UE-3e\#4 (p1) & Aleman & $<0.3$ & $<4.0$ & $<20.0$ & $<37.0$ & $<0.3$ & $<0.3$ & $<0.1$ & $<10.0$ & $<1.0$ \\
\hline UE-3e\#4 (p1) & Aleman & $<0.3$ & $<4.0$ & 20.0 & $<37.0$ & $<0.3$ & $<0.3$ & $<0.1$ & $<10.0$ & 2.0 \\
\hline UE-7ns & Bourbon & $<0.3$ & $<4.0$ & 20.0 & $<37.0$ & $<0.3$ & $<0,3$ & 0.5 & $<10.0$ & $<10$ \\
\hline
\end{tabular}


TABLE IV

Cavity or Near-Cavity "Hot" Well Analyses

1977-1992

\begin{tabular}{|c|c|c|c|c|c|c|c|c|c|c|c|c|c|}
\hline Well name & Event & Depth & $\begin{array}{c}\text { Collection } \\
\text { date }\end{array}$ & Date Sampled & HNO3/ filter & DO & $\mathrm{pH}$ & $T$ & TDS & Alkalinity & $\mathrm{Li}$ & B & $\mathrm{Na}$ \\
\hline Unit & & $(f t)$ & decay & date collected & & $(\mathrm{mg} / \mathrm{L})$ & & & $(\mu g / g)$ & (mg CaCO3/L) & $m g / L)$ & $m g / L)$ & $m g / L$ \\
\hline Half-life (a) & & & correct date & in field & & & & & & & & & \\
\hline \multicolumn{14}{|l|}{ Ref. date } \\
\hline U-3cn PS\#2 & Bilby & pump & 30-Sep-77 & 27-Sep-77 & no / no & & & & & & & & \\
\hline U-3cn PS\#2 & Bilby & pump & $30-\operatorname{Sep}-77$ & 29-Sep-77 & no/ no & & & & & & & & \\
\hline U-3cn PS\#2 & Bilby & pump & 30-Sep-77 & 29-Sep-77 & no/no & & & & & & & & \\
\hline $\mathrm{U}-3 \mathrm{cn}$ PS\#2 & Bilby & pump & $30-$ Sep-77 & 29-Sep-77 & no / no & & & & & & & & \\
\hline U-3cn PS\#2 & Bilby & pump & 30-Sep-77 & 29-Sep-77 & no / no & & & & & & & & \\
\hline U-3cn PS\#2 & Bilby & pump & 30-Sep-77 & 29-Sep-77 & no / no & & & & & & & & \\
\hline U-3cn PS\#2 & Bilby & pump & $30-5 \oplus p-77$ & 30-Sep-77 & no/ no & & & & 432 & & & & \\
\hline U-3cn PS\#2 & Bilby & pump & 30-Sep-77 & 21-Oct-81 & no / no & & & & 341 & & & & \\
\hline U-3cn $\# 5$ & Bilby & pump & 30-Sep-77 & $21-0 \mathrm{ct}-80$ & $\mathrm{no} / 1 \mu \mathrm{m}$ & & & & 393 & & & & \\
\hline U-3cn $\# 5$ & Bilby & pump & 30-Sep-77 & $20-$ Oct-81 & no/ 1 $14 \mathrm{~m}$ & & & & 304 & & & & \\
\hline & & & & & & & & & & & & & \\
\hline UE-2ce & Nash & pump & 15-Mar-78 & 15-Mar-78 & no $/ 1 \mu \mathrm{m}$ & & 6.9 & 33 & 609 & & nd & & 51 \\
\hline UE-2ce & Nash & pump & 15-Mar-78 & $9-0 \mathrm{ct}-80$ & no $/ 1 \mu \mathrm{m}$ & & & & 354 & & & & \\
\hline UE-2ce & Nash & pump & 15-Mar-78 & 9-Jul-82 & $\mathrm{no} / 1 \mu \mathrm{m}$ & & & & 535 & & & & \\
\hline UE-2ce & Nash & pump & 15-Mar-78 & 10-Aug-83 & no $/ 0.45 \mu \mathrm{m}$ & & 7.5 & & 442 & & nd & & 46 \\
\hline UE-2ce & Nash & pump & 15-Mar-78 & 18-Apr-84 & $\mathrm{no} / 1 \mu \mathrm{m}$ & & 6.6 & 33 & 567 & & 0.17 & & 63 \\
\hline & & & & & & & & & & & & & \\
\hline RNM-1 & Cambric & pump & 14-May-79 & 4-Sep-79 & no / no & & & & 532 & & & & \\
\hline RNM-1 & Cambric & pump & 14-May-79 & $5-0 \mathrm{ct}-81$ & no / no & & & & 228 & & & & \\
\hline RNM-1 & Cambric & pump & 14-May-79 & 1-Apr-82 & no/ no & & & & 232 & & & & \\
\hline RNM-1 & Cambric & $?$ & 10-Apr-85 & 10-Apr-85 & $\mathrm{no} / 0.2 \mu \mathrm{m}$ & & & & & & & & \\
\hline RNM-1 & Cambric & pump & 7-May-86 & 7-May-86 & no / no & & & & & & & & \\
\hline RNM-2S & Cambric & pump & 14-May-79 & 29-Nov-78 & no / no & & & & 790 & & & & \\
\hline RNM-2S & Cambric & pump & 14-May-79 & 7 -Feb-79 & no / no & & & & 579 & & & & \\
\hline RNM-2S & Cambric & pump & 14-May-79 & 14-Mar-79 & no / no & & & & 565 & & & & \\
\hline RNM-2S & Cambric & pump & 14-May-79 & 29-Aug-79 & no/ $/ \mu \mathrm{m}$ & & & & 389 & & & & \\
\hline RNM-2S & Cambric & pump & 14-May-79 & 17-Apr-80 & $\mathrm{no} / 1 \mu \mathrm{m}$ & & & & 458 & & & & \\
\hline RNM-2S & Cambric & pump & 14-May-79 & 23-Jul-80 & no $/ 1 \mu \mathrm{m}$ & & & & 282 & & & & \\
\hline RNM-2S & Cambric & pump & 14-May-79 & 22-Jul-81 & $\mathrm{no} / 1 \mu \mathrm{m}$ & & & & 290 & & & & \\
\hline RNM-2S** & Cambric & pump & 14-May-79 & 10-Nov-82 & no $/ 1 \mu \mathrm{m}$ & & 8.1 & & 262 & & & & 61 \\
\hline
\end{tabular}




\section{TABI.E IV}

\section{Cavity or Near-Cavity "Hot" Well Analyses}

1977-1992

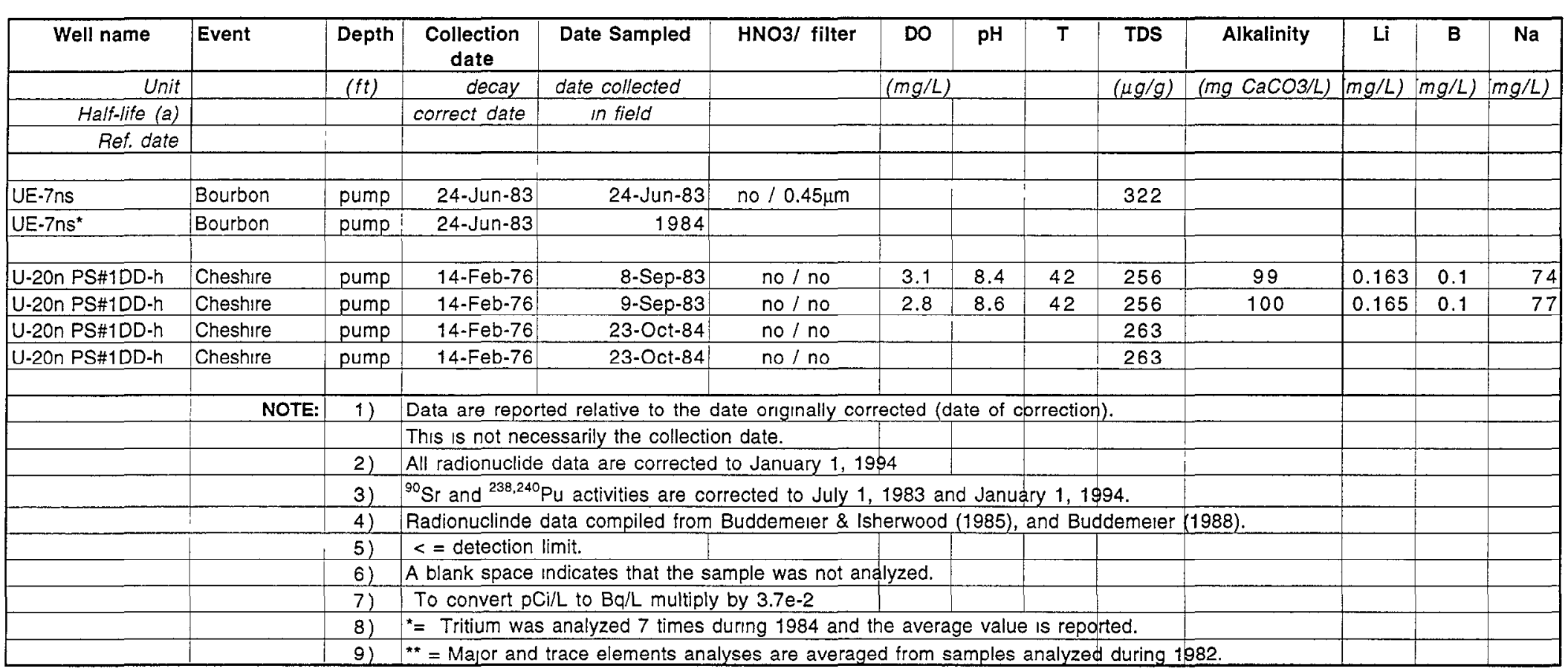




\section{TABLE IV}

Cavity or Near-Cavity "Hot" Well Analyses 1977-1992

\begin{tabular}{|c|c|c|c|c|c|c|c|c|c|c|c|c|c|c|c|c|c|}
\hline Well name & $\mathrm{Mg}$ & Al & Si & $P$ & $\mathrm{Cl}$ & $\mathrm{K}$ & $\mathrm{Ca}$ & $M n$ & $\mathrm{Fe}$ & Co & $\mathrm{Ni}$ & $\mathrm{Cu}$ & $\mathrm{Zn}$ & Se & Sr & Mo & $\mathrm{Pb}$ \\
\hline Unit & $m g / L)$ & $(m g / L)$ & $m g / L$ & $m g / L)$ & $m g / L)$ & $m g / L$ & $m g / L)$ & $m g / L)$ & $m g / L)$ & $m g / L)$ & $m g / L)$ & $m g / L)$ & $m g / L)$ & $m g / L)$ & $m g / L)$ & $m g / L)$ & $m g / L)$ \\
\hline \multicolumn{18}{|c|}{\begin{tabular}{|l|l|l|l|l|l|l|l|l|} 
Half-life (a) & & & & & & & \\
\end{tabular}} \\
\hline \multicolumn{18}{|l|}{ Ref. date } \\
\hline \multicolumn{18}{|l|}{ U-3cn PS\#2 } \\
\hline \multicolumn{18}{|l|}{ U-3cn PS\#2 } \\
\hline \multicolumn{18}{|l|}{ U-3cn PS\#2 } \\
\hline \multicolumn{18}{|l|}{ U-3cn PS\#2 } \\
\hline \multicolumn{18}{|l|}{ U-3cn PS\#2 } \\
\hline \multicolumn{18}{|l|}{ U-3en PS\#2 } \\
\hline \multicolumn{18}{|l|}{ U-3cn PS\#2 } \\
\hline \multicolumn{18}{|l|}{ U-3cn PS\#2 } \\
\hline \multicolumn{18}{|l|}{ U-3en PS\#2 } \\
\hline \multicolumn{18}{|l|}{ U-3cn \#5 } \\
\hline \multicolumn{18}{|l|}{ U-3cn \#5 } \\
\hline & & & & & & & & & & & & & & & & & \\
\hline UE-2ce & 44.0 & & & & 103 & 34.0 & 97.0 & & & & & & & & & & \\
\hline \multicolumn{18}{|l|}{ UE-2Ce } \\
\hline \multicolumn{18}{|l|}{ UE-2ce } \\
\hline \multicolumn{18}{|l|}{ UE-2ce } \\
\hline UE-2ce & 31.4 & & & & 49.2 & 22.1 & 70.9 & & & & & & & & & & \\
\hline UE-2ce & 39.9 & & & & 108.8 & 22.3 & 94.7 & & & & & & & & 0.27 & & \\
\hline & & & & & & & & & & & & & & & & & \\
\hline \multicolumn{18}{|l|}{ RNM-1 } \\
\hline \multicolumn{18}{|l|}{ RNM-1 } \\
\hline \multicolumn{18}{|l|}{ RNM-1 } \\
\hline \multicolumn{18}{|l|}{ RNM-1 } \\
\hline \multicolumn{18}{|l|}{ RNM-1 } \\
\hline \multicolumn{18}{|l|}{ RNM-2S } \\
\hline RNM-2S & & & & & & & & & & & & & & & & & \\
\hline RNM-2S & & & & & & & & & & & & & & & & & \\
\hline RNM-2S & & & & & & & & & & & & & & & & & \\
\hline RNM-2S & & & & & & & & & & & & & & & & & \\
\hline RNM-2S & & & & & & & & & & & & & & & & & \\
\hline RNM-2S & & & & & & & & & & & & & & & & & \\
\hline RNM-2S** & 5.5 & & & & 15 & 9.9 & 18.0 & & & & & & & & & & \\
\hline RNM-2S & & & & & & & & & & & & & & & & & \\
\hline
\end{tabular}


TABLE IV

Cavity or Near-Cavity "Hot" Well Analyses

1977-1992

\begin{tabular}{|c|c|c|c|c|c|c|c|c|c|c|c|c|c|c|c|c|c|}
\hline Well name & $\mathrm{Mg}$ & Al & $\mathrm{Si}$ & $\mathbf{P}$ & $\mathrm{Cl}$ & $\mathrm{K}$ & $\mathrm{Ca}$ & $\mathrm{Mn}$ & $\mathrm{Fe}$ & Co & $\mathrm{Ni}$ & $\mathrm{Cu}$ & $\mathrm{Zn}$ & $\mathrm{Se}$ & $\mathrm{Sr}$ & Mo & $\mathrm{Pb}$ \\
\hline Unit & $m g / L$ & $m g / L)$ & $(m g / L)$ & $(m g / L)$ & $(m g / L)$ & $m g / L$ & $m g / L)$ & $m g / L$ & $(m g / L)$ & $m g / L)$ & $m g / L)$ & $(m g / L)$ & $m g / L$ & $m g / L)$ & $m g / L)$ & $m g / L)$ & $m g / L)$ \\
\hline \multicolumn{18}{|c|}{ Half-life (a) } \\
\hline \multicolumn{18}{|l|}{ Ref date } \\
\hline & & & & & & & & & & & & & & & & & \\
\hline \multicolumn{18}{|l|}{ UE-7ns } \\
\hline \multicolumn{18}{|l|}{ UE-7ns ${ }^{*}$} \\
\hline & & & & & & & & & & & & & & & & & \\
\hline U-20n PS\#1DD-h & 0.2 & 0.98 & 29.7 & 0.114 & 13.4 & 2.4 & 1.9 & 0.08 & 0.17 & 0.00 & 0.003 & 0.003 & 0.00 & 0.05 & 0.005 & 0.019 & 0.019 \\
\hline U-20n PS\#1DD-h & 0.1 & 2.14 & 33.1 & 0.122 & 14 & 3.1 & 1.7 & 0.10 & 0.27 & 0.00 & 0.003 & 0.003 & 0.01 & 0.05 & 0.002 & 0.021 & 0.022 \\
\hline \multicolumn{18}{|l|}{ U-20n PS\#1DD-h } \\
\hline U-20n PS\#1DD-h & & & & & & & & & & & & & & & & & \\
\hline & & & & & & & & & & & & & & & & & \\
\hline
\end{tabular}


TABLE IV

Cavity or Near-Cavity "Hot" Well Analyses

1977-1992

\begin{tabular}{|c|c|c|c|c|c|c|c|c|c|c|c|}
\hline Well name & $3 \mathrm{H}$ & $3 \mathrm{H}$ & $3 \mathrm{H}$ & $22 \mathrm{Na}$ & $22 \mathrm{Na}$ & $22 \mathrm{Na}$ & $40 K$ & $40 \mathrm{~K}$ & $40 \mathrm{~K}$ & $54 \mathrm{Mn}$ & $54 M n$ \\
\hline Unit & $(d p m / L)$ & $(\rho \mathrm{Ci} / L)$ & $(p C i / L)$ & $(d p m / L)$ & $(p C i / L)$ & $(\rho \mathrm{CI} / \mathrm{L})$ & $(d p m / L)$ & $(p C i / L)$ & $(p C i / L)$ & $(p \mathrm{Ci} / L)$ & $(\rho \mathrm{Ci} / \mathrm{L})$ \\
\hline Half-life (a) & 12.26 & 12.26 & 12.26 & & 2.61 & 2.61 & & $1.24 E+9$ & $1.24 E+9$ & 0.85 & 0.85 \\
\hline Ref. date & & collect. & $1 / 1 / 94$ & collect. & collect. & $1 / 1 / 94$ & collect. & collect. & $1 / 1 / 94$ & collect. & $1 / 1 / 94$ \\
\hline U-3cn PS\#2 & $7.77 \mathrm{E}+07$ & $3.50 \mathrm{e}+7$ & $140 \mathrm{e}+7$ & $<2.00 e-3$ & $<9.01 e-4$ & $<1.19 \mathrm{e}-5$ & $4.84 e+1$ & $2.18 e+1$ & $2.18 \mathrm{e}+1$ & & \\
\hline U-3cn PS\#2 & $7.93 E+07$ & $3.57 e+7$ & $142 e+7$ & $\leq 2.00 \mathrm{e}-3$ & $<9.01 \mathrm{e}-4$ & $<1.19 e-5$ & $4.60 e+1$ & $2.07 e+1$ & $2.07 e+1$ & & \\
\hline U-3cn PS\#2 & $8.12 \mathrm{E}+07$ & $3.66 e+7$ & $146 e+7$ & $<2.00 \mathrm{e}-3$ & $<9.01 \mathrm{e}-4$ & $<1.19 e-5$ & $4.66 e+1$ & $2.10 e+1$ & $2.10 \mathrm{e}+1$ & & \\
\hline U-3cn PS\#2 & $7.90 \mathrm{E}+07$ & $3.56 e+7$ & $1.42 e+7$ & $<2.00 \mathrm{e}-3$ & $<9.01 \mathrm{e}-4$ & $<1.19 \mathrm{e}-5$ & $2.84 \mathrm{e}+1$ & $1.28 e+1$ & $1.28 \mathrm{e}+1$ & & \\
\hline U-3en PS\#2 & $7.59 \mathrm{E}+07$ & $3.42 e+7$ & $1.36 e+7$ & $<2.00 e-3$ & $<9.01 \mathrm{e}-4$ & $<1.19 e-5$ & $5.79 e+1$ & $2.61 e+1$ & $2.61 e+1$ & & \\
\hline U-3cn PS\#2 & $7.83 \mathrm{E}+07$ & $3.53 e+7$ & $1.41 \theta+7$ & $<2.00 e-3$ & $<9.01 e-4$ & $<1.19 e-5$ & $2.88 e+1$ & $1.30 e+1$ & $1.30 e+1$ & & \\
\hline U-3cn PS\#2 & $7.73 E+07$ & $3.48 e+7$ & $1.39 e+7$ & $<2.00 e-3$ & $<9.01 e-4$ & $<119 e-5$ & $3.24 e+1$ & $1.46 e+1$ & $1.46 \mathrm{e}+1$ & & \\
\hline U-3cn PS\#2 & $7.73 E+07$ & $3.48 e+7$ & $1.39 e+7$ & $<2.00 e-3$ & $<9.01 \mathrm{e}-4$ & $<119 e-5$ & $3.56 e+1$ & $1.60 e+1$ & $1.60 \theta+1$ & & \\
\hline U-3en PS\#2 & $8.14 \mathrm{E}+07$ & $3.67 e+7$ & $1.46 e+7$ & $<2.00 e-3$ & $<9.01 e-4$ & $<1.19 \mathrm{e}-5$ & $2.84 e+1$ & $1.28 e+1$ & $1.28 \mathrm{e}+1$ & & \\
\hline$U-3 c n \# 5$ & 19.51 & $8.79 e+0$ & $3.51 \mathrm{e}+0$ & $<2.00 e-3$ & $<9.01 \mathrm{e} .4$ & $<1.19 \mathrm{e}-5$ & $1.60 \mathrm{e}+1$ & $7.21 \mathrm{e}+0$ & $7.21 e+0$ & & \\
\hline U-3cn \#5 & 5.16 & $2.32 e+0$ & $9.27 e-1$ & $<2.00 \mathrm{e}-3$ & $<9.01 e-4$ & $<1.19 e-5$ & $1.34 \mathrm{e}+1$ & $6.01 e+0$ & $6.01 \mathrm{e}+0$ & & \\
\hline UE-2ce & $2.80 \mathrm{E}+07$ & $1.26 e+7$ & $5.16 \mathrm{e}+6$ & $6.80 \mathrm{e}-1$ & $3.06 \mathrm{e}-1$ & $4.57 e-3$ & $4.19 e+1$ & $1.89 e+1$ & $1.89 e+1$ & & \\
\hline UE-2ce & & & & $3.90 \mathrm{e}-1$ & $1.76 \mathrm{e}-1$ & $2.62 e-3$ & $2.35 e+1$ & $1.06 e+1$ & $1.06 e+1$ & & \\
\hline UE-2ce & $4.80 E+06$ & $2.16 \mathrm{e}+6$ & $8.85 e+5$ & $1.40 \mathrm{e}-1$ & $6.31 \mathrm{e}-2$ & $9.42 e-4$ & $3.24 \theta+1$ & $146 \theta+1$ & $146 e+1$ & & \\
\hline UE-2ce & $4.40 \mathrm{E}+07$ & $1.98 e+7$ & $8.11 e+6$ & $9.80 \mathrm{e}-1$ & $4.41 e-1$ & $6.59 e-3$ & $4.09 e+1$ & $1.84 e+1$ & $1.84 e+1$ & & \\
\hline UE-2ce & $3.92 E+07$ & $1.77 e+7$ & $7.23 e+6$ & $111 e+0$ & $5.00 e-1$ & $747 e-3$ & $2.49 \mathrm{e}+1$ & $1.12 e+1$ & $1.12 \mathrm{e}+1$ & & \\
\hline UE-2Ce & $7.28 \mathrm{E}+07$ & $3.28 e+7$ & $1.34 e+7$ & $1.97 e+0$ & $8.87 e-1$ & $1.33 e-2$ & $4.08 e+1$ & $1.84 \mathrm{e}+1$ & $1.84 e+1$ & & \\
\hline & & & & & & & & & & & \\
\hline RNM-1 & $3.20 \mathrm{E}+06$ & $1.44 \mathrm{e}+6$ & $6.30 e+5$ & $<4.00 e-3$ & $<1.80 e-3$ & $<3.67 \mathrm{e}-5$ & $1.52 e+1$ & $6.86 e+0$ & $6.86 e+0$ & & \\
\hline RNM-1 & $1.37 E+05$ & $6.17 e+4$ & $2.70 e+4$ & $<4.00 e-3$ & $<1.80 \mathrm{e}-3$ & $<3.67 \mathrm{e}-5$ & $113 e+1$ & $5.09 \theta+0$ & $5.09 e+0$ & & \\
\hline RNM-1 & $7.20 \mathrm{E}+04$ & $3.24 \mathrm{e}+4$ & $142 \theta+4$ & $<4.00 e-3$ & $<1.80 e-3$ & $<3.67 e-5$ & $1.33 e+1$ & $5.97 \mathrm{e}+0$ & $5.97 \mathrm{e}+0$ & & \\
\hline \multicolumn{12}{|l|}{ RNM-1 } \\
\hline \multicolumn{12}{|l|}{ RNM-1 } \\
\hline RNM-2S & $1.80 E+06$ & $8.11 e+5$ & $3.54 e+5$ & $<4.00 e-3$ & $<1.80 e-3$ & $<3.67 e-5$ & $2.24 e+1$ & $1.01 e+1$ & $1.01 e+1$ & & \\
\hline RNM-2S & $2.40 \mathrm{E}+06$ & $1.08 e+6$ & $4.73 e+5$ & $<4.00 \mathrm{e}-3$ & $<1.80 \mathrm{e}-3$ & $<3.67 \mathrm{e}-5$ & $1.61 \mathrm{e}+1$ & $7.27 e+0$ & $7.27 \mathrm{e}+0$ & & \\
\hline RNM-2S & $2.63 E+06$ & $1.18 e+6$ & $5.18 e+5$ & $<4.00 e-3$ & $<1.80 e-3$ & $<3.67 \mathrm{e}-5$ & $1.73 e+1$ & $7.78 \mathrm{e}+0$ & $7.78 \mathrm{e}+0$ & & \\
\hline RNM-2S & $4.23 E+06$ & $1.91 e+6$ & $8.33 e+5$ & $<4.00 e-3$ & $<1.80 \mathrm{e}-3$ & $<3.67 e-5$ & $2.29 e+1$ & $1.03 e+1$ & $1.03 e+1$ & & \\
\hline RNM-2S & $6.10 \mathrm{E}+06$ & $2.75 e+6$ & $1.20 \mathrm{e}+6$ & $<4.00 \mathrm{e}-3$ & $<1.80 \mathrm{e}-3$ & $<3.67 \mathrm{e}-5$ & $2.86 e+1$ & $1.29 \mathrm{e}+1$ & $1.29 e+1$ & & \\
\hline RNM-2S & $6.24 \mathrm{E}+06$ & $2.81 e+6$ & $1.23 e+6$ & $<4.00 e-3$ & $<1.80 e-3$ & $<3.67 e-5$ & $1.89 \mathrm{e}+1$ & $8.52 e+0$ & $8.52 e+0$ & & \\
\hline RNM-2S & $6.02 E+06$ & $2.71 e+6$ & $1.19 e+6$ & $<4.00 \mathrm{e}-3$ & $<1.80 e-3$ & $<3.67 e-5$ & $1.56 e+1$ & $7.04 e+0$ & $7.04 e+0$ & & \\
\hline RNM-2S** & $6.24 \mathrm{E}+06$ & $2.81 e+6$ & $1.23 e+6$ & $<4.00 e-3$ & $<1.80 e-3$ & $<3.67 e-5$ & $1.80 e+1$ & $8.10 e+0$ & $8.10 e+0$ & & \\
\hline RNM-2S & $4.73 E+06$ & $2.13 e+6$ & $9.31 e+5$ & $<4.00 e-3$ & $<1.80 e-3$ & $<3.67 e-5$ & $1.82 e+1$ & $8.22 e+0$ & $8.22 e+0$ & & \\
\hline
\end{tabular}


TABLE IV

Cavity or Near-Cavity "Hot" Well Analyses

1977-1992

\begin{tabular}{|c|c|c|c|c|c|c|c|c|c|c|c|}
\hline Well name & $3 \mathrm{H}$ & $3 \mathrm{H}$ & $3 \mathrm{H}$ & $22 \mathrm{Na}$ & $22 \mathrm{Na}$ & $22 \mathrm{Na}$ & $40 \mathrm{~K}$ & $40 K$ & $40 \mathrm{~K}$ & $54 \mathrm{Mn}$ & $54 \mathrm{Mn}$ \\
\hline Unit & $(d p m / L)$ & $(p C i / L)$ & $(p C i / L)$ & $(d p m / L)$ & $(p \mathrm{Ci} / \mathrm{L})$ & $(p C i / L)$ & $(d p m / L)$ & $(p C i / L)$ & $(p C i / L)$ & $(p C i / L)$ & $(p C i / L)$ \\
\hline Half-life (a) & 12.26 & 12.26 & 12.26 & & 2.61 & 2.61 & & $1.24 E+9$ & $1.24 E+9$ & 0.85 & 0.85 \\
\hline Ref. date & & collect. & $1 / 1 / 94$ & collect. & collect. & $1 / 1 / 94$ & collect. & collect. & $1 / 1 / 94$ & collect. & $1 / 1 / 94$ \\
\hline UE-7ns & $<3.00 \mathrm{e}-3$ & $<1.35 \mathrm{e}-3$ & $<7.45 \mathrm{e}-4$ & $<2.00 e-3$ & $<9.01 e-4$ & $<5.48 e-5$ & $1.10 e+1$ & $4.96 e+0$ & $4.96 e+0$ & & \\
\hline UE-7ns* & $4.81 e+3$ & $2.17 e+3$ & $1.19 \mathrm{e}+3$ & & & & & & & & \\
\hline & & & & & & & & & & & \\
\hline U-20n PS\#1DD-h & & $6.15 \mathrm{e}+8$ & $2.24 e+8$ & & $7.57 e+0$ & $6 . \overline{50 e-2}$ & & $2.67 e+0$ & $2.67 e+0$ & $1.18 \mathrm{e}+2$ & $5.49 e-5$ \\
\hline U-20n PS\#1DD-h & & $6.14 e+8$ & $2.23 e+8$ & & $9.79 e+0$ & $8.40 \mathrm{e}-2$ & & $5.24 e+0$ & $5.24 e+0$ & $1.20 \mathrm{e}+2$ & $5.58 \mathrm{e} .5$ \\
\hline U-20n PS\#1DD-h & & $5.04 e+8$ & $1.83 e+8$ & & $8.02 e+0$ & $6.88 \mathrm{e}-2$ & & $3.58 \mathrm{e}+0$ & $3.58 e+0$ & $9.64 e+1$ & $4.48 \mathrm{e}-5$ \\
\hline U-20n PS\#1DD-h & & $5.04 e+8$ & $1.83 e+8$ & & $8.50 e+0$ & $7.30 \mathrm{e}-2$ & & $4.15 \theta+0$ & $4.15 e+0$ & $3.31 \theta+1$ & $1.54 e-5$ \\
\hline
\end{tabular}


TABLE IV

Cavity or Near-Cavity "Hot" Well Analyses

1977-1992

\begin{tabular}{|c|c|c|c|c|c|c|c|c|c|c|c|c|}
\hline Well name & $60 \mathrm{Co}$ & $60 \mathrm{Co}$ & $60 \mathrm{Co}$ & $\begin{array}{c}\text { Coll date for } \\
\text { Sr Pu }\end{array}$ & $90 \mathrm{Sr}$ & $90 \mathrm{Sr}$ & 106Ru & 106Ru & 106Ru & $125 \mathrm{Sb}$ & $125 \mathrm{Sb}$ & $125 \mathrm{Sb}$ \\
\hline Unit & $(d p m / L)$ & $(p C i / L)$ & $(p C i / L)$ & & $(p C i / L)$ & $(p C i / L)$ & $(d p m / L)$ & $(p \subset i / L)$ & $(\rho \mathrm{Ci} / \mathrm{L})$ & $(d p m / L)$ & $(p C i / L)$ & $(p C i / L)$ \\
\hline Half-life (a) & 5.27 & 5.27 & 5.27 & & & 29 & & 1.02 & 1.02 & 2.76 & 2.76 & 2.76 \\
\hline Ref date & collect. & collect. & $1 / 1 / 94$ & & $7 / 1 / 83$ & $1 / 1 / 94$ & collect. & collect. & $1 / 1 / 94$ & collect. & collect. & $1 / 1 / 94$ \\
\hline U-3cn PS\#2 & $6.80 e-1$ & $3.06 \mathrm{e}-1$ & $3.61 \mathrm{e}-2$ & & & & $<2.20 e+0$ & $<9.91 e-1$ & $<1.58 \mathrm{e}-5$ & $1.90 e+0$ & $8.56 \mathrm{e}-1$ & $1.44 \mathrm{e}-2$ \\
\hline U-3cn PS\#2 & $6.90 \mathrm{e}-1$ & $3.11 \mathrm{e}-1$ & $3.67 e-2$ & & & & $<2.40 e+0$ & $<1.08 \mathrm{e}+0$ & $<1.72 \mathrm{e}-5$ & $2.30 e+0$ & $1.04 e+0$ & $1.74 \mathrm{e}-2$ \\
\hline U-3cn PS\#2 & $6.60 e-1$ & $2.97 \mathrm{e}-1$ & $3.51 e-2$ & & & & $<2.40 e+0$ & $<1.08 \theta+0$ & $<1.72 \mathrm{e}-5$ & $2.30 e+0$ & $1.04 e+0$ & $1.74 \mathrm{e}-2$ \\
\hline U.3cn PS\#2 & $<1.70 \mathrm{e}-1$ & $<7.66 \mathrm{e}-2$ & $<9.03 e-3$ & & & & $<1.800+0$ & $<8.11 \mathrm{e}-1$ & $<1.29 \mathrm{e}-5$ & $1.50 e+0$ & $6.76 \mathrm{e}-1$ & $1.14 \mathrm{e}-2$ \\
\hline U-3cn PS\#2 & $6.20 \mathrm{e}-1$ & $2.79 \mathrm{e}-1$ & $3.29 e-2$ & & & & $<1.50 e+0$ & $<6.76 \mathrm{e}-1$ & $<1.08 e-5$ & $2.80 e+0$ & $1.26 e+0$ & $2.12 e-2$ \\
\hline$U-3 \mathrm{cn}$ PS\#2 & $<1.80 \mathrm{e}-1$ & $<8.11 \mathrm{e}-2$ & $<9.56 e-3$ & & & & $<1.80 e+0$ & $<8.11 \mathrm{e}-1$ & $<1.29 e-5$ & $<6.10 \mathrm{e}-1$ & $<2.75 \mathrm{e}-1$ & $<4.62 e-3$ \\
\hline U-3cn PS\#2 & $<2.70 \mathrm{e}-1$ & $<1.22 \mathrm{e}-1$ & $<1.43 \mathrm{e}-2$ & & & & $<2.00 e+0$ & $<9.01 e-1$ & $<1.44 \mathrm{e}-5$ & $140 e+0$ & $6.31 \mathrm{e}-1$ & $1.06 e-2$ \\
\hline U-3cn PS\#2 & $1.50 \mathrm{e}-1$ & $6.76 \mathrm{e}-2$ & $7.97 e-3$ & & & & $3.90 e+0$ & $1.76 e+0$ & $2.80 e-5$ & $2.10 e+0$ & $9.46 \mathrm{e}-1$ & $1.59 \mathrm{e}-2$ \\
\hline U-3cn PS\#2 & $3.00 e-2$ & $1.35 \mathrm{e}-2$ & $1.59 e-3$ & 1-Jul-83 & $5.66 \mathrm{e}-2$ & $4.40 e-2$ & $<710 \mathrm{e}-1$ & $<3.20 e-1$ & $<5.10 e-6$ & $6.00 e-1$ & $2.70 \mathrm{e}-1$ & $4.55 e-3$ \\
\hline U. $3 \mathrm{cn} \# 5$ & $<3.00 \mathrm{e}-3$ & $<1.35 \mathrm{e}-3$ & $<1.59 \mathrm{e}-4$ & 1-Jul-83 & $4.33 e-3$ & $3.37 e-3$ & $<4.00 \mathrm{e}-2$ & $<1.80 e-2$ & $<2.87 \mathrm{e}-7$ & $<1.00 \mathrm{e}-2$ & $<4.50 \mathrm{e}-3$ & $<7.580-5$ \\
\hline U-3cn \#5 & $<3.00 \mathrm{e}-3$ & $<1.35 \mathrm{e}-3$ & $<1.59 \mathrm{e}-4$ & $1-\mathrm{Jul}-83$ & $2.94 e-3$ & $2.29 e-3$ & $<7.00 \mathrm{e}-2$ & $<3.15 e-2$ & $<5.03 e-7$ & $<1.00 e-2$ & $<4.50 e-3$ & $<7.58 \mathrm{e}-5$ \\
\hline UE-2ce & $<2.00 e-3$ & $<9.01 \mathrm{e}-4$ & $<1.13 e-4$ & & & & $<2.00 \mathrm{e}-1$ & $<9.01 e-2$ & $<1.96 \mathrm{e}-6$ & $<3.00 e-2$ & $<1.35 \mathrm{e}-2$ & $<2.55 e-4$ \\
\hline UE-2ce & $<2.00 e-3$ & $<9.01 \mathrm{e}-4$ & $<113 e-4$ & $1-\mathrm{Ju} /-83$ & $5.98 e-1$ & $4.65 e-1$ & $<5.00 e-1$ & $<2.25 e-1$ & $<4.89 \mathrm{e}-6$ & $<2.00 e-2$ & $<9.01 \mathrm{e}-3$ & $<1.70 \mathrm{e}-4$ \\
\hline UE-2ce & $<2.00 \theta-3$ & $<9.01 e-4$ & $<1.13 e-4$ & 1-Jul-83 & $4.93 e-1$ & $3.84 e-1$ & $<2.00 e-1$ & $<9.01 \mathrm{e}-2$ & $<1.96 \mathrm{e}-6$ & $<2.00 e-2$ & $<9.01 \mathrm{e}-3$ & $<1.70 e-4$ \\
\hline UE-2Ce & $<2.00 e-3$ & $<9.01 \mathrm{e}-4$ & $<1.13 \mathrm{e}-4$ & & & & $<1.30 \mathrm{e}+0$ & $<5.86 e-1$ & $<1.27 \mathrm{e}-5$ & $<2.00 e-2$ & $<9.01 \mathrm{e}-3$ & $<1.70 e-4$ \\
\hline UE-2ce & $3.10 \mathrm{e}-1$ & $140 \mathrm{e}-1$ & $1.75 e-2$ & & & & $<6.00 e-2$ & $<2.70 e-2$ & $<5.87 \mathrm{e}-7$ & $<1.20 \mathrm{e}-1$ & $<5.41 \mathrm{e}-2$ & $<1.02 e-3$ \\
\hline UE-2ce & $<9.00 e-3$ & $<4.05 e-3$ & $<5.08 \mathrm{e}-4$ & & & & $<6.00 \mathrm{e}-2$ & $<2.70 e-2$ & $<5.87 e-7$ & $<9.000-2$ & $<4.05 \mathrm{e}-2$ & $<7.64 \mathrm{e}-4$ \\
\hline & & & & & & & & & & & & \\
\hline RNM-1 & $<2.00 e-3$ & $<9.01 e-4$ & $<1.31 e-4$ & & & & $<2.70 \mathrm{e}-1$ & $<1.22 \mathrm{e}-1$ & $<5.83 \mathrm{e}-6$ & $2.60 e-1$ & $1.17 \mathrm{e}-1$ & $2.96 e-3$ \\
\hline RNM-1 & $<2.00 e-3$ & $<9.01 e-4$ & $<1.31 \mathrm{e}-4$ & $1-J u / .83$ & $1 . \overline{30 e}+1$ & $1.01 e+1$ & $<2.80 \mathrm{e}-1$ & $<1.26 e-1$ & $<6.04 \mathrm{e}-6$ & $2.00 e-1$ & $9.01 e-2$ & $2.28 e-3$ \\
\hline RNM-1 & $4.00 e-2$ & $1.80 \mathrm{e}-2$ & $2.63 \mathrm{e}-3$ & & & & $<6.30 \mathrm{e}-1$ & $<2.84 \mathrm{e}-1$ & $<1.36 \mathrm{e}-5$ & $<7.00 \mathrm{e}-2$ & $<3.15 \mathrm{e}-2$ & $<7.97 e-4$ \\
\hline \multicolumn{13}{|l|}{ RNM-1 } \\
\hline \multicolumn{13}{|l|}{ RNM-1 } \\
\hline BNM-2S & $<2.00 e-3$ & $<9.01 e-4$ & $<1.31 \mathrm{e}-4$ & & & & $1.60 e-1$ & $7.21 e-2$ & $3.45 \mathrm{e}-6$ & $<2.00 \mathrm{e}-2$ & $<9.01 e-3$ & $<2.28 \mathrm{e}-4$ \\
\hline RNM-2S & $<2.00 e-3$ & $<9.01 e .4$ & $<1.31 \mathrm{e}-4$ & & & & $1.60 \mathrm{e}-1$ & $7.21 \mathrm{e}-2$ & $3.45 \mathrm{e}-6$ & $<2.00 e-2$ & $<9.01 e-3$ & $<2.28 \mathrm{e}-4$ \\
\hline RNM-2S & $<2.00 \mathrm{e}-3$ & $<9.01 e-4$ & $<1.31 \mathrm{e}-4$ & & & & $1.50 \mathrm{e}-1$ & $6.76 \mathrm{e}-2$ & $3.24 e-6$ & $<2.00 \mathrm{e}-2$ & $<9.01 e-3$ & $<2.28 \mathrm{e}-4$ \\
\hline RNM-2S & $<2.00 e-3$ & $<9.01 \mathrm{e}-4$ & $<1.31 \mathrm{e}-4$ & & & & $3.10 \mathrm{e}-1$ & $1.40 \mathrm{e}-1$ & $6.69 \mathrm{e}-6$ & $1.00 e-2$ & $4.50 e-3$ & $1.14 \mathrm{e}-4$ \\
\hline RNM-2S & $<2.00 e-3$ & $<9.01 \mathrm{e}-4$ & $<1.31 \mathrm{e}-4$ & & & & $1.80 \mathrm{e}-1$ & $8.11 e-2$ & $3.88 \mathrm{e}-6$ & $<1.00 \mathrm{e}-2$ & $<4.50 e-3$ & $<1.14 \mathrm{e}-4$ \\
\hline RNM-2S & $<2.00 e-3$ & $<9.01 e-4$ & $<1.31 e-4$ & & & & $2.20 \mathrm{e}-1$ & $9.91 e-2$ & $4.75 e-6$ & $<1.00 \mathrm{e}-2$ & $<4.50 e-3$ & $<1.14 \mathrm{e}-4$ \\
\hline RNM-2S & $<2.00 e-3$ & $<9.01 e-4$ & $<1.31 e-4$ & & & & $<2.00 \theta-2$ & $<9.01 e-3$ & $<4.32 \mathrm{e}-7$ & $<1.00 \mathrm{e}-2$ & $<4.50 e-3$ & $<1.14 \mathrm{e}-4$ \\
\hline RNM-2S** & $<2.00 e-3$ & $<9.01 e .4$ & $<1.31 \mathrm{e}-4$ & $1-\mathrm{Ju} / \mathrm{-}-83$ & $1.53 e-2$ & $1.19 \mathrm{e}-2$ & $<1.00 e-2$ & $<4.50 \mathrm{e}-3$ & $<2.16 \mathrm{e}-7$ & $<1.00 e-2$ & $<4.50 \mathrm{e}-3$ & $<1.14 e-4$ \\
\hline RNM-2S & $<2.00 e-3$ & $<9.01 e-4$ & $<1.31 \mathrm{e}-4$ & & & & $<2.00 e-2$ & $<9.01 e-3$ & $<4.320-7$ & $<3.00 e-2$ & $<1.35 \mathrm{e}-2$ & $<3.41 e-4$ \\
\hline
\end{tabular}


TABLE IV

Cavity or Near-Cavity "Hot" Well Analyses

1977-1992

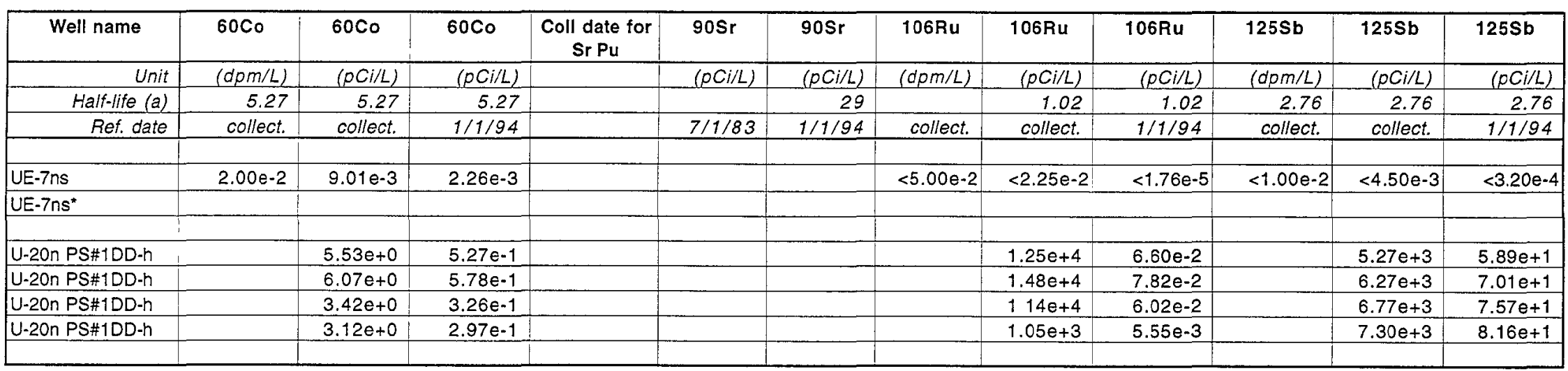


TABLE IV

Cavity or Near-Cavity "Hot" Well Analyses

1977-1992

\begin{tabular}{|c|c|c|c|c|c|c|c|c|c|c|c|}
\hline Well name & $134 \mathrm{Cs}$ & $134 \mathrm{Cs}$ & $137 \mathrm{Cs}$ & $137 \mathrm{Cs}$ & $137 \mathrm{Cs}$ & $144 \mathrm{Ce}$ & $144 \mathrm{Ce}$ & 152Eu & $152 \mathrm{Eu}$ & $154 E \mathrm{u}$ & $154 \mathrm{Eu}$ \\
\hline Unit & $(p \mathrm{Ci} / \mathrm{L})$ & $(p C i / L)$ & $(d p m / L)$ & $(p C i / L)$ & $(p \mathrm{Ci} / \mathrm{L})$ & $(p C i / L)$ & $(p C i / L)$ & $(p C i / L)$ & $(p C i / L)$ & $(\rho C i / L)$ & $(p C i / L)$ \\
\hline Half-life (a) & 2.07 & 2.07 & 30.17 & 30.17 & 30.17 & & 0.78 & 13.4 & 13.4 & 8.5 & 8.5 \\
\hline Ref. date & collect. & $1 / 1 / 94$ & collect. & collect. & $1 / 1 / 94$ & collect. & $1 / 1 / 94$ & collect. & $1 / 1 / 94$ & collect. & $1 / 1 / 94$ \\
\hline U-3cn PS\#2 & & & $7.20 e+0$ & $3.24 e+0$ & $2.23 e+0$ & & & & & & \\
\hline U-3cn PS\#2 & & & $7.40 e+0$ & $3.33 e+0$ & $2.29 e+0$ & & & & & & \\
\hline U-3cn PS\#2 & & & $8.30 e+0$ & $3.74 e+0$ & $2.57 e+0$ & & & & & & \\
\hline U-3cn PS\#2 & & & $5.98 e+0$ & $2.69 \mathrm{e}+0$ & $1.85 e+0$ & & & & & & \\
\hline U-3cn PS\#2 & & & $9.60 e+0$ & $4.32 e+0$ & $2.98 e+0$ & & & & & & \\
\hline U-3cn PS\#2 & & & $6.60 e+0$ & $2.97 e+0$ & $2.05 e+0$ & & & & & & \\
\hline U-3cn PS\#2 & & & $6.60 e+0$ & $2.97 e+0$ & $2.05 e+0$ & & & & & & \\
\hline U-3cn PS\#2 & & & $6.70 e+0$ & $3.02 e+0$ & $2.08 e+0$ & & & & & & \\
\hline U-3cn PS\#2 & & & $3.60 e+0$ & $1.62 e+0$ & $1.12 \mathrm{e}+0$ & & & & & & \\
\hline$U-3$ cn $\# 5$ & & & $6.00 \theta-3$ & $2.70 \theta-3$ & $1.86 \theta-3$ & & & & & & \\
\hline U-3cn \#5 & & & $<3.00 e-3$ & $<1.35 e-3$ & $<9.30 e-4$ & & & & & & \\
\hline & & & & & & & & & & & \\
\hline UE-2ce & & & $<1.00 \mathrm{e}-2$ & $<4.50 \mathrm{e}-3$ & $<3.13 e-3$ & & & & & & \\
\hline UE-2ce & & & $2.00 e-2$ & $9.01 e-3$ & $6.27 e-3$ & & & & & & \\
\hline UE-2ce & & & $4.00 e-2$ & $1.80 e-2$ & $1.25 e-2$ & & & & & & \\
\hline UE-2ce & & & $3.00 e-2$ & $1.35 e-2$ & $9.40 \mathrm{e}-3$ & & & & & & \\
\hline UE-2ce & & & $1.40 \mathrm{e}-1$ & $6.31 e-2$ & $4.39 \mathrm{e}-2$ & & & & & & \\
\hline UE-2ce & & & $2.00 e-2$ & $9.01 e-3$ & $6.27 e-3$ & & & & & & \\
\hline & & & & & & & & & & & \\
\hline RNM-1 & & & $8.45 e+1$ & $3.80 e+1$ & $2.72 e+1$ & & & & & & \\
\hline RNM-1 & & & $3.74 e+1$ & $1.68 \mathrm{e}+1$ & $1.20 e+1$ & & & & & & \\
\hline RNM-1 & & & $3.71 e+1$ & $1.67 \mathrm{e}+1$ & $1.19 e+1$ & & & & & & \\
\hline RNM-1 & & & & & & & & & & & \\
\hline RNM-1 & & & & & & & & & & & \\
\hline RNM-2S & & & $4.00 e-3$ & $1.80 e-3$ & $1.29 e-3$ & & & & & & \\
\hline RNM-2S & & & $<6.00 e-3$ & $<2.70 \mathrm{e}-3$ & $<1.93 e-3$ & & & & & & \\
\hline RNM-2S & & & $<6.00 e-3$ & $<2.70 \mathrm{e}-3$ & $<1.93 \mathrm{e}-3$ & & & & & & \\
\hline RNM-2S & & & $1.00 \mathrm{e}-2$ & $4.50 \mathrm{e}-3$ & $3.22 e-3$ & & & & & & \\
\hline RNM-2S & & & $2.00 e-2$ & $9.01 \mathrm{e}-3$ & $6.44 e-3$ & & & & & & \\
\hline RNM-2S & & & $1.00 \mathrm{e}-2$ & $4.50 \mathrm{e}-3$ & $3.22 \mathrm{e}-3$ & & & & & & \\
\hline RNM-2S & & & $3.00 \mathrm{e}-2$ & $1.35 \mathrm{e}-2$ & $9.65 e-3$ & & & & & & \\
\hline RNM-2S $S^{\star \star}$ & & & $2.00 \mathrm{e}-2$ & $9.01 \mathrm{e}-3$ & $6.44 e-3$ & & & & & & \\
\hline RNM-2S & & & $3.00 e-3$ & $1.35 \mathrm{e}-3$ & $9.65 e-4$ & & & & & & \\
\hline
\end{tabular}


TABLE IV

Cavity or Near-Cavity "Hot" Well Analyses

1977-1992

\begin{tabular}{|c|c|c|c|c|c|c|c|c|c|c|c|}
\hline Well name & $134 \mathrm{Cs}$ & $134 \mathrm{Cs}$ & $137 \mathrm{Cs}$ & $137 \mathrm{Cs}$ & $137 \mathrm{Cs}$ & $144 \mathrm{Ce}$ & $144 \mathrm{Ce}$ & 152Eu & 152Eu & 154Eu & 154Eu \\
\hline Unit & $(p C i / L)$ & $(p C i / L)$ & $(d p m / L)$ & $(p C i / L)$ & $(p C i / L)$ & $(p C i / L)$ & $(p C i / L)$ & $(p C i / L)$ & $(0 C i / L)$ & $(p C i / L)$ & $(p C i / L)$ \\
\hline Half-life (a) & 2.07 & 2.07 & 30.17 & 30.17 & 30.17 & & 0.78 & 13.4 & 13.4 & 8.5 & 8.5 \\
\hline Ref. date & collect. & $1 / 1 / 94$ & collect. & collect. & $1 / 1 / 94$ & collect. & $1 / 1 / 94$ & collect. & $1 / 1 / 94$ & collect. & $1 / 1 / 94$ \\
\hline UE-7ns & & & $9.00 e-2$ & $4.05 e-2$ & $3.18 \mathrm{e}-2$ & & & & & & \\
\hline \multicolumn{12}{|l|}{ UE-7ns ${ }^{*}$} \\
\hline & & & & & & & & & & & \\
\hline U-20n PS\#1DD-h & $2.68 e+1$ & $6.63 e-2$ & & $3.40 e+3$ & $2.25 e+3$ & $149 e+1$ & $1.87 e-6$ & $1.20 e+1$ & $4.76 e+0$ & $1.64 e+1$ & $3.82 e+0$ \\
\hline U.20n PS\#1DD-h & $2.70 e+1$ & $6.68 \mathrm{e}-2$ & & $4.60 e+3$ & $3.05 e+3$ & $1.01 e+1$ & $1.27 e-6$ & $7.52 e+0$ & $2.98 e+0$ & $1.31 \mathrm{e}+1$ & $3.05 e+0$ \\
\hline U-20n PS\#1DD-h & $2.67 e+1$ & $6.61 e-2$ & & $4.39 e+3$ & $2.91 e+3$ & $9.56 \mathrm{e}+0$ & $1.20 \mathrm{e}-6$ & $6.75 e+0$ & $2.68 \mathrm{e}+0$ & $1.17 e+1$ & $2.72 e+0$ \\
\hline
\end{tabular}




\section{TABLE IV}

\section{Cavity or Near-Cavity "Hot" Well Analyses}

1977-1992

\begin{tabular}{|c|c|c|c|c|c|c|c|c|c|c|c|}
\hline Well name & $155 \mathrm{Eu}$ & 155Eu & 155Eu & $235 \mathrm{U}$ & $235 \mathrm{U}$ & $235 \mathrm{U}$ & $238 U$ & $238 \mathrm{U}$ & $238 \mathrm{U}$ & $239,240 \mathrm{Pu}$ & $239,240 \mathrm{Pu}$ \\
\hline Unit & $(d p m / L)$ & $(p C i / L)$ & $(p C i / L)$ & $(d p m / L)$ & $(p C i / L)$ & $(\rho C i / L)$ & $(d p m / L)$ & $(p C i / L)$ & $(p C i / L)$ & $(p C i / L)$ & $(\rho \mathrm{Ci} / \mathrm{L})$ \\
\hline Half-life (a) & & 4.73 & 4.73 & & $7.04 E+8$ & $7.04 E+8$ & & $4.46 E+9$ & $4.46 E+9$ & 6560 & 6560 \\
\hline Ref. date & collect. & collect. & $1 / 1 / 94$ & collect. & collect. & $1 / 1 / 94$ & collect. & collect. & $1 / 1 / 94$ & $7 / 1 / 83$ & $1 / 1 / 94$ \\
\hline U-3cn PS\#2 & $<4.00 e-3$ & $<1.80 \mathrm{e}-3$ & $<1.66 \mathrm{e}-4$ & $<1.70 \mathrm{e}-1$ & $<7.66 \mathrm{e}-2$ & $<7.66 \mathrm{e}-2$ & $6.00 e+1$ & $2.70 e+1$ & $2.70 e+1$ & & \\
\hline U-3cn PS\#2 & $<4.00 e-3$ & $<1.80 e-3$ & $<1.66 \mathrm{e}-4$ & $1.80 e+0$ & $8.11 e-1$ & $8.11 \theta-1$ & $4.78 e+1$ & $2.15 e+1$ & $2.15 e+1$ & $8.56 \mathrm{E}+01$ & $8.55 e+1$ \\
\hline U-3cn PS\#2 & $<4.00 e-3$ & $<1.80 e-3$ & $<1.66 \mathrm{e}-4$ & $1.90 \mathrm{e}+0$ & $8.56 e-1$ & $8.56 \mathrm{e}-1$ & $5.63 e+1$ & $2.53 e+1$ & $2.53 e+1$ & & \\
\hline U-3cn PS\#2 & $<4.00 e-3$ & $<1.80 e-3$ & $<1.66 \mathrm{e}-4$ & $5.00 e-1$ & $2.25 e-1$ & $2.25 \mathrm{e}-1$ & $<1.84 e+0$ & $<8.29 \mathrm{e}-1$ & $<8.29 e-1$ & & \\
\hline U-3en PS\#2 & $<4.00 e-3$ & $<1.80 e-3$ & $<1.66 \mathrm{e}-4$ & $1.90 e+0$ & $8.56 e-1$ & $8.56 \mathrm{e}-1$ & $5.80 e+1$ & $2.61 e+1$ & $2.61 e+1$ & & \\
\hline U-3en PS\#2 & $<4.00 e-3$ & $<1.80 e-3$ & $<1.66 \mathrm{e}-4$ & $<1.70 \mathrm{e}-1$ & $<7.66 \mathrm{e}-2$ & $<7.66 \mathrm{e}-2$ & $<1.84 \mathrm{e}+0$ & $<8.29 \mathrm{e}-1$ & $<8.29 \mathrm{e}-1$ & & \\
\hline U-3cn PS\#2 & $<4.00 e-3$ & $<1.80 e-3$ & $<1.66 e-4$ & $<1.70 \mathrm{e}-1$ & $<7.66 \mathrm{e}-2$ & $<7.66 \mathrm{e}-2$ & $<1.84 e+0$ & $<8.29 \mathrm{e}-1$ & $<8.29 \mathrm{e}-1$ & & \\
\hline U-3cn PS\#2 & $<4.00 e-3$ & $<1.80 e-3$ & $<1.66 \mathrm{e}-4$ & $6.00 \mathrm{e}-1$ & $2.70 e-1$ & $2.70 \mathrm{e}-1$ & $1.48 e+1$ & $6.64 \mathrm{e}+0$ & $6.64 e+0$ & & \\
\hline U-3cn PS\#2 & $<4.00 e-3$ & $<1.80 e-3$ & $<1.66 \mathrm{e}-4$ & $4.00 \mathrm{e}-1$ & $1.80 e-1$ & $1.80 e-1$ & $8.98 e+0$ & $4.05 e+0$ & $4.05 e+0$ & $2.35 \mathrm{E}-03$ & $2.35 e-3$ \\
\hline U-3cn \#5 & $2.00 \mathrm{e}-1$ & $9.010-2$ & $8.320-3$ & $1.00 \mathrm{e}-1$ & $4.50 e-2$ & $4.50 e-2$ & $1.31 e+0$ & $5.90 \mathrm{e}-1$ & $5.90 e-1$ & $2.34 \mathrm{E}-04$ & $2.34 c-4$ \\
\hline U-3en \#5 & $2.00 e-1$ & $9.01 e-2$ & $8.32 e-3$ & $8.00 e-2$ & $3.60 e-2$ & $3.60 e-2$ & $1.09 e+0$ & $4.91 \mathrm{e}-1$ & $4.91 \mathrm{e}-1$ & $2.11 \mathrm{E}-04$ & $2.11 e-4$ \\
\hline UE-2ce & $1.20 \mathrm{e}-1$ & $5.41 \mathrm{e}-2$ & $5.34 e-3$ & $2.20 \mathrm{e}-1$ & $9.91 e-2$ & $9.91 e-2$ & $4.17 e+0$ & $1.88 e+0$ & $1.88 \mathrm{e}+0$ & & \\
\hline UE-2ce & $7.00 \mathrm{e}-2$ & $3.15 \mathrm{e}-2$ & $3.11 e-3$ & $1.50 \mathrm{e}-1$ & $6.76 \mathrm{e}-2$ & $6.76 \mathrm{e}-2$ & $3.96 \mathrm{e}+0$ & $1.78 e+0$ & $1.78 \mathrm{e}+0$ & & \\
\hline UE-2ce & $1.00 \mathrm{e}-1$ & $4.50 e-2$ & $4.45 e-3$ & $1.50 \mathrm{e}-1$ & $6.76 \mathrm{e}-2$ & $6.76 e-2$ & $3.12 e+0$ & $141 e+0$ & $1.41 \mathrm{e}+0$ & & \\
\hline UE-2ce & $4.00 e-2$ & $1.80 \mathrm{e}-2$ & $1.78 e-3$ & $2.80 e-1$ & $1.26 \mathrm{e}-1$ & $1.26 e-1$ & $2.13 e+0$ & $9.59 \mathrm{e}-1$ & $9.59 e-1$ & & \\
\hline UE-2ce & $3.60 \mathrm{e}-1$ & $1.62 \mathrm{e}-1$ & $1.60 \mathrm{e}-2$ & $110 \mathrm{e}-1$ & $4.95 e-2$ & $4.95 e-2$ & $2.28 e+0$ & $1.03 e+0$ & $1.03 e+0$ & & \\
\hline UE-2ce & $3.80 \mathrm{e}-1$ & $1.71 \mathrm{e}-1$ & $1.69 e-2$ & $1.90 \mathrm{e}-1$ & $8.56 e-2$ & $8.56 e-2$ & $4.15 e+0$ & $1.87 e+0$ & $1.87 e+0$ & & \\
\hline RNM-1 & $<4.00 e-3$ & $<1.80 e \cdot 3$ & $<2.11 \mathrm{e}-4$ & $3.20 \mathrm{e}-1$ & $1.44 \mathrm{e}-1$ & $1.44 \mathrm{e}-1$ & $4.63 e+0$ & $2.09 e+0$ & $2.09 e+0$ & & \\
\hline RNM-1 & $<4.00 \mathrm{e}-3$ & $<1.80 e-3$ & $<2.11 \mathrm{e}-4$ & $1.50 \mathrm{e}-1$ & $6.76 \mathrm{e}-2$ & $6.76 \mathrm{e}-2$ & $2.79 e+0$ & $1.26 e+0$ & $1.26 \mathrm{e}+0$ & $3.05 E-03$ & $3.05 e-3$ \\
\hline RNM-1 & $<4.00 \mathrm{e}-3$ & $<1.80 \mathrm{e}-3$ & $<2.11 \mathrm{e}-4$ & $1.80 e-1$ & $8.11 \mathrm{e}-2$ & $8.11 e-2$ & $3.82 \mathrm{e}+0$ & $1.72 e+0$ & $1.72 \mathrm{e}+0$ & & \\
\hline \multicolumn{12}{|l|}{ RNM-1 } \\
\hline \multicolumn{12}{|l|}{ RNM-1 } \\
\hline RNM-2S & $<4.00 \mathrm{e}-3$ & $<1.80 e-3$ & $<2.11 \mathrm{e}-4$ & $2.00 \mathrm{e}-1$ & $9.01 e-2$ & $9.01 e-2$ & $5.55 e+0$ & $2.50 e+0$ & $2.50 \mathrm{e}+0$ & & \\
\hline RNM-2S & $<4.00 \mathrm{e}-3$ & $<1.80 \mathrm{e} \cdot 3$ & $<2.11 e-4$ & $1.20 \mathrm{e}-1$ & $5.41 \mathrm{e}-2$ & $5.41 \mathrm{e}-2$ & $2.49 e+0$ & $1.12 e+0$ & $1.12 e+0$ & & \\
\hline RNM-2S & $<4.00 e-3$ & $<1.80 e-3$ & $<2.11 e-4$ & $1.70 \mathrm{e}-1$ & $7.66 \mathrm{e}-2$ & $7.66 \mathrm{e}-2$ & $3.55 e+0$ & $1.60 e+0$ & $1.60 \mathrm{e}+0$ & & \\
\hline RNM-2S & $<4.00 \mathrm{e}-3$ & $<1.80 \mathrm{e}-3$ & $<2.11 \mathrm{e}-4$ & $9.00 e-2$ & $4.05 \mathrm{e}-2$ & $4.05 e-2$ & $1.90 \mathrm{e}+0$ & $8.56 e-1$ & $8.56 \mathrm{e}-1$ & & \\
\hline RNM-2S & $<4.000 .3$ & $<1.80 e-3$ & $<2.11 \mathrm{e}-4$ & $3.00 e-1$ & $1.35 e-1$ & $1.35 e-1$ & $7.37 e+0$ & $3.32 e+0$ & $3.32 e+0$ & $!$ & \\
\hline RNM-2S & $<4.00 e-3$ & $<1.80 \mathrm{e}-3$ & $<2.11 \mathrm{e}-4$ & $1.90 e-1$ & $8.56 e-2$ & $8.56 e-2$ & $4.73 e+0$ & $2.13 e+0$ & $2.13 e+0$ & & \\
\hline RNM-2S & $<4.00 \mathrm{e}-3$ & $<1.80 \mathrm{e}-3$ & $<2.11 \mathrm{e}-4$ & $1.30 \mathrm{e}-1$ & $5.86 \mathrm{e}-2$ & $5.86 e-2$ & $2.81 e+0$ & $1.27 e+0$ & $1.27 e+0$ & & \\
\hline RNM-2S** & $<4.00 \mathrm{e}-3$ & $<1.80 \mathrm{e}-3$ & $<2.11 \mathrm{e}-4$ & $1.50 \mathrm{e}-1$ & $6.76 \mathrm{e}-2$ & $6.76 e-2$ & $2.50 \mathrm{e}+0$ & $1.13 e+0$ & $1.13 e+0$ & $1.83 \mathrm{E}-04$ & $1.83 e-4$ \\
\hline RNM-2S & $<4.00 e-3$ & $<1.80 \mathrm{e}-3$ & $<2.11 \mathrm{e}-4$ & $1.80 \mathrm{e}-1$ & $8.11 e-2$ & $8.11 e-2$ & $2.44 \mathrm{e}+0$ & $1.10 \mathrm{e}+0$ & $1.10 \mathrm{e}+0$ & & \\
\hline
\end{tabular}


TABLE IV

Cavity or Near-Cavity "Hot" Well Analyses

1977-1992

\begin{tabular}{|c|c|c|c|c|c|c|c|c|c|c|c|}
\hline Well name & 155Eu & 155Eu & 155Eu & $235 \mathrm{U}$ & $235 \mathrm{U}$ & $235 \mathrm{U}$ & $238 U$ & $238 U$ & $238 \mathrm{U}$ & $239,240 \mathrm{Pu}$ & $239,240 \mathrm{Pu}$ \\
\hline Unit & $(d p m / L)$ & $(p C i / L)$ & $(p \mathrm{Ci} / \mathrm{L})$ & $(d p m / L)$ & $(p C i / L)$ & $(p C i / L)$ & $(d p m / L)$ & $(p C i / L)$ & $(p C i / L)$ & $(p C i / L)$ & $(p C i / L)$ \\
\hline Half-life (a) & & 4.73 & 4.73 & & $7.04 E+8$ & $7.04 E+8$ & & $4.46 E+9$ & $4.46 E+9$ & 6560 & 6560 \\
\hline Ref. date & collect. & collect. & $1 / 1 / 94$ & collect. & collect. & $1 / 1 / 94$ & collect. & collect. & $1 / 1 / 94$ & $7 / 1 / 83$ & $1 / 1 / 94$ \\
\hline UE-7ns & $<4.00 e-3$ & $<1.80 e-3$ & $<3.85 e-4$ & $7.00 e-3$ & $3.15 \mathrm{e}-3$ & $3.15 e-3$ & $<2.70 e-1$ & $<1.22 \mathrm{e}-1$ & $<1.22 e-1$ & & \\
\hline \multicolumn{12}{|l|}{ UE-7ns ${ }^{\star}$} \\
\hline & & & & & & & & & & & \\
\hline U-20n PS\#1DD-h & & $4.10 e+1$ & $2.98 e+0$ & & & & & & & & \\
\hline U-20n PS\#1DD-h & & $6.10 e+1$ & $4.44 e+0$ & & & & & & & & \\
\hline U-20n PS\#1DD-h & & $3.87 e+1$ & $2.82 e+0$ & & & & & & & & \\
\hline U-20n PS\#1DD-h & & $3.45 e+1$ & $2.51 e+0$ & & & & & & & & \\
\hline & & & & & & & & & & & \\
\hline
\end{tabular}




\section{CHAPTER 5}

\section{BATCH LEACHING OF NUCLEAR EXPLOSIVE MELT DEBRIS \\ David K. Smith}

\section{INTRODUCTION}

Radionuclides from nuclear explosive melt debris are released to the environment under saturated conditions. Knowledge of which radionuclides are in solution, their relative abundance, and the controls on their mobility in part defines radionuclide transport and the resulting dose to potential downgradient receptors. The radiologic source term includes all radionuclides residual after a nuclear explosion; the hydrologic source term includes only those radionuclides soluble or otherwise available for transport by groundwater (Kersting, 1996). Knowledge of the transition between the radiologic and hydrologic source term is critical. If the hydrologic source term can be better predicted through credible statements about the release of radionuclides to solution, potentially impacted groundwater resources can be managed more effectively.

The hydrologic source term may be investigated through direct measurement of contaminated groundwater or numerical predictions coupled with experimental studies. While empirical measure is always preferred, the ability to collect representative water samples from the vicinity of nuclear tests detonated greater than $600 \mathrm{~m}$ below the surface is an expensive, technically complex enterprise. The predictive approach involves estimates of the release of radionuclides from the dissolution of debris residual from the explosion.

Between 700 and 1300 tons of glass are produced for each kiloton of yield of an underground nuclear explosion. Glass is produced from 1) the condensation of plasma produced by the vaporization of the device and surrounding media at the time of the explosion, 2) shock melting the result of the high early-time pressures and temperatures accompanying growth of the cavity, and 3) melting of host rock adjacent to the standing cavity wall (Borg et al., 1976). This melt collects on the bottom of cavity forming a puddle. The composition of the melt is similar to the composition of the host rock in the vicinity of the explosion (Schwartz et al., 1984; Smith, 1995). Once the pressure in the expanding cavity reaches the prevailing lithostatic pressure, the cavity reaches its maximum size. High thermal gradients produced away from the edge of the cavity causes ablation of the wall material which falls into the still molten puddle. Larger pieces survive intact within or above the glass. As the internal pressure drops (to a few bars) eventually the cavity can no longer support the overburden and the cavity collapses forming a rubble chimney. Where rock strengths are low, the chimney may collapse to the surface. It is from this 
heterogeneous matrix of glass and collapse rubble in the vicinity of the working (firing) point that samples were taken for the present study.

Borg et al. (1976) and Smith (1993) reviewed experimental data on the leaching of nuclear explosive melt debris. An earlier study by Essington and Sharp (1968) studied the 2 day and 56 day equilibration of debris from the 1957 RAINIER with synthetic sodium bicarbonate groundwater to investigate the nature of radionuclide release and dependence of particle size. Coles et al. (1978) reported initial 120 day results of a single pass leaching of a single nuclear explosive melt glass using bicarbonate groundwater from well $5 \mathrm{~B}$ at the Nevada Test Site; Failor et al. (1983) reports on the final results of the same experiment for a 420 day leaching trial for three melt glasses. Batch leaching experiments of nuclear explosive melt debris using bicarbonate water from NTS well J-13 and deionized water has been reported by Thompson (1990, 1991, and 1994).

\section{EXPERIMENTAL}

An emphasis of the present study was to investigate the leaching of both vitreous and crystalline matrices residual from a nuclear explosion. Additionally, a priority was to monitor changes in leachate chemistry indicative of hydration, hydrolysis, ion-exchange, or network dissolution. Earlier studies had keyed on leaching only the black glass that collected on the floor of the puddle which incorporated the refractory radionuclides with higher boiling points (e,g., $\left.{ }^{155} \mathrm{Eu},{ }^{144} \mathrm{Ce},{ }^{90} \mathrm{Sr},{ }^{239} \mathrm{Pu}\right)$. Borg (1975) reports that only 2 to $3 \%$ of ${ }^{144} \mathrm{Cc}$ is lost during the explosion from traditional "puddle" samples "in which other refractories are equally well retained.". However, field investigations have clearly shown that volatile long-lived radionuclides with lower boiling points (e.g., ${ }^{60} \mathrm{Co},{ }^{90} \mathrm{Sr},{ }^{125} \mathrm{Sb}$, ${ }^{137} \mathrm{Cs}$ ) clearly fractionate from the puddle region and may be encountered within and adjacent to the cavity (Smith et al., 1996) or within the event chimney (Thompson, 1996). Other studies indicate that concentrations of volatile radionuclides are higher where associated with larger specific-surface areas created during the explosion (Esser, 1995). Some of these radionuclides are long-lived, are easily mobilized, and are important to comprehensive studies of radionuclide migration. For this reason, debris from four different tests was leached. Two of the samples included black glass typical of "meltglass" produced by an underground nuclear explosion; the remaining samples consisted of crystalline material collected within the cavity-chimney region which were characterized by high-levels of total gamma activity.

Batch leaching was used and followed the procedure described by Thompson (1990) with some variation. 

1) Each of the four debris samples were obtained from bulk samples returned from postshot drilling. A representative sample was selected from each and ground to pass through a 60 mesh screen $(<250 \mu \mathrm{m})$. Fines were not removed from the samples.

2) Each sample was then characterized by X-ray diffraction for constituent mineralogy and examined by electron microscopy to determine the major element composition as well as the range in particle diameters.

3) Approximately 1 gram of sample was next weighed into a screw-cap centrifuge tube with $20 \mathrm{ml}$ of water from NTS Water Well 4A.

4) The rock and water was put on a shaker table and shaken at a constant temperature of $25^{\circ} \mathrm{C}$. Each of the four samples were shaken for a periods of 8 days, 31 days, 156 days, and 391 days.

5) At the end of each run the samples were withdrawn from the shaker table and centrifuged to separate the fluid and solid phase. Fluid was extracted with a syringe and filtered through a $0.45 \mu \mathrm{m}$ filter using a syringe filter support.

6) The filtrate was analyzed for gamma-emitting radionuclides by gamma spectroscopy, for major cations ( $\mathrm{Ca}, \mathrm{Mg}, \mathrm{Na}, \mathrm{K}, \mathrm{Si}, \mathrm{Al}, \mathrm{Fe}$ ) by ICP-AES, and for $\mathrm{pH}$ using a calibrated $\mathrm{pH}$ ion-selective electrode.

7) A split was taken of the leached particulate for polished grain mounts and analysis by scanning electron microscopy. In this way, cross-sections of grains could be interrogated to study reaction surfaces.

\section{PRE-LEACH RESULTS}

\section{Sample Locations and Zero-time Activities}

The four core samples were collected from four different events; three were fired beneath Yucca Flat and one fired beneath Pahute Mesa. Samples were collected at different heights from the bottom of the melt puddle determined at the time of post-shot drilling. Table 1 summarizes the location and bulk zero-time activities of the samples. 
TABLE I

Sample Location Above Bottom of Puddle (m)

and Zero-time Activity (mrem)

\begin{tabular}{lllll}
\hline & Core \#1 & Core\#2 & Core \#6 & Core \#8 \\
\hline & 31 & 19 & 11 & 10 \\
Height Above Puddle Bottom (m) & 700 & 10,000 & 400 & 11,000 \\
Zero-timc Activity (mrcm) & & &
\end{tabular}

Matrix Characterization, X-Ray Diffraction, Energy Dispersive Analysis, and Particle Sizing

Core samples \#1 and \#8 are composed of glass produced from the melting of the explosion and cores \#2 and \#6 being composed of a mineral breccia incorporated from the cavity and chimney incompletely melted by the explosion. In particular, core \#6 is characterized by calcite and dolomite in addition to quartz, feldspars, and magnetite. X-ray diffraction analyses indicate each matrix is distinct. Results are compiled in Table II. Order in the table is not meant to imply absolute abundance of constituent minerals or glass.

TABLE II

X-ray Diffraction Results

\begin{tabular}{cccc}
\hline Core 1 & Core 2 & Core 6 & Core 8 \\
\hline Glass & K-Na Feldspars & Quartz & Glass \\
Sepolite & Quartz & Na-Ca Feldspars & K-Na Feldspars \\
Magnetite & Na-Ca Feldspars & K-Feldspars & \\
& Magnetite & Magnetite & \\
& & Calcite \\
& Dolomite & \\
\hline
\end{tabular}

Energy dispersive analysis using the electron microprobe with a $40,000 \mu \mathrm{m}^{2}$ raster area indictes that the grains of core \#1, \#2, and \#8 are largely composed of $\mathrm{SiO}_{2}(>\sim 80$ weight percent), with $\mathrm{Al}_{2} \mathrm{O}_{3}$, with lesser amounts of $\mathrm{K}_{2} \mathrm{O}, \mathrm{CaO}$, and $\mathrm{FeO}$ (combined $<\sim 20$ weight percent). Single grains analysis of Core 6 indicates a minority of the grains are enriched in $\mathrm{CaO}$ and $\mathrm{MgO}$ (to $\sim 40$ weight percent) while the remainder are composed of $\mathrm{SiO}_{2}$ and $\mathrm{Al}_{2} \mathrm{O}_{3}$.

Geometric surface areas were calculated assuming cubic particle shapes. Particle diameters were estimated from electron imaging from the less than 60 mesh $(<250 \mu \mathrm{m})$ size fraction. Calculated surface areas are listed in Table III. 
TABLE III

Calculated Surface Area

\begin{tabular}{lllll}
\hline & Core \#1 & Core \#2 & Core \#6 & Core \#8 \\
\hline & & & & \\
Particle Diameter $(\mu \mathrm{m})$ & 60 & 40 & 40 & 50 \\
Particle Density $\left(\mathrm{g} / \mathrm{cm}^{3}\right)$ & 2.3 & 2.3 & 2.3 & 2.3 \\
Surface Area $\left(\mathrm{m}^{2} / \mathrm{g}\right)$ & 0.072 & 0.109 & 0.109 & 0.087 \\
\hline
\end{tabular}

\section{Leaching Solution}

The water for the leaching experiment was obtained from Water Well 4A in Area 6 at the Nevada Test Site. This well was selected because it is completed in bedded and ash-flow tuff aquifers beneath the Yucca Flat testing area. Because this well is a pumped water supply well, a representative sample could easily be obtained. Analyses of the unfiltered water indicate it is typical of dilute bicarbonate groundwaters encountered at the Nevada Test Site; results are compiled in Table IV.

TABLE IV

Analysis of Unfiltered Water from Water Well 4A in mg/L

\begin{tabular}{cccc}
\hline Cations & \multicolumn{3}{c}{$\begin{array}{c}\text { Anions and } \\
\mathrm{pH}\end{array}$} \\
\hline $\mathrm{Ca}$ & 24 & $\mathrm{~F}$ & 0.747 \\
$\mathrm{Mg}$ & 7.4 & $\mathrm{Cl}^{-}$ & 11.1 \\
$\mathrm{Na}$ & 51 & $\mathrm{Br}^{-}$ & $<0.04$ \\
$\mathrm{~K}$ & 5.4 & $\mathrm{NO}_{3}^{-}$ & 15.7 \\
$\mathrm{Si}$ & 30 & $\mathrm{PO}_{4}^{-}$ & $<0.06$ \\
$\mathrm{Al}$ & 0.0065 & $\mathrm{SO}_{4}^{-2}$ & 37.6 \\
$\mathrm{Fe}$ & 0.0018 & $\mathrm{HCO}_{3}^{-}$ & 168 \\
& & $\mathrm{pH}^{-}$ & 7.72 \\
\hline
\end{tabular}

\section{Radiochemistry of the Nuclear Explosive Melt Debris}

Tritium, fission products, activation products, and actinides are incorporated in the nuclear explosive melt debris residual from an underground nuclear explosion. Radionuclides with higher boiling points (e.g., $\mathrm{Zr}, \mathrm{Ce}, \mathrm{Eu}$ ) condense at higher temperatures and become volumetrically incorporated in the melt glass; radionuclides with lower boiling points and gaseous precursors (e.g., $\mathrm{Na}, \mathrm{Sb}, \mathrm{Ru}, \mathrm{Cs}$ ) are volatile and may be transported with noncondensible gases away from the working point where they condense on cooler fracture surfaces particularly those created during the collapse of the nuclear test chimney. On a field-scale, fractionation of radionuclides between glass at the level of the working point 
and volatile radionuclides in the chimney has been documented by Thompson (1996). Gamma-counting of radionuclides associated with the four core samples indicates partitioning of volatile and refractory radionuclides. Cores \#1 and \#8 preferentially incorporate rare earth elements and transition metals into the glass matrix. Volatile radionuclides are noted in all cores; in particular, ${ }^{137} \mathrm{Cs}$ which has a gaseous precursor is enriched in the non-glassy breccia cores \#2 and \#6. Gamma analysis of the four core samples is presented in Table V; results are presented in DPM / gram corrected to event zero (firing) time.

TABLE V

Gamma Analyses of Core Sample in DPM / gram Corrected to Event Zero-time

\begin{tabular}{|c|c|c|c|c|}
\hline & $\begin{array}{l}\text { Core \#1 } \\
\text { glass }\end{array}$ & $\begin{array}{l}\text { Core \#2 } \\
\text { breccia } \\
\end{array}$ & $\begin{array}{l}\text { Core \#6 } \\
\text { breccia }\end{array}$ & $\begin{array}{l}\text { Core \#8 } \\
\text { glass }\end{array}$ \\
\hline${ }^{22} \mathrm{Na}$ & $1.17 \mathrm{E}+4$ & & $1.23 E+2$ & $4.48 E+2$ \\
\hline${ }^{22} \mathrm{Na}$ Error & $5.80 \mathrm{E}+0$ & & $3.21 \mathrm{E}+1$ & $4.33 \mathrm{E}+1$ \\
\hline${ }^{40} \mathrm{~K}$ & & & $510 \mathrm{E}+1$ & \\
\hline${ }^{40} \mathrm{~K}$ Error & & & $4.25 \mathrm{E}+1$ & \\
\hline${ }^{54} \mathrm{Mn}$ & $6.29 E+6$ & & $3.10 \mathrm{E}+4$ & $3.75 E+5$ \\
\hline${ }^{54} \mathrm{Mn}$ Error & $6.60 \mathrm{E}+0$ & & $1.19 \mathrm{E}+1$ & $2.70 \mathrm{E}+0$ \\
\hline${ }^{60} \mathrm{Co}$ & $1.18 \mathrm{E}+5$ & $209 E+2$ & $9.77 E+2$ & $6.96 \mathrm{E}+3$ \\
\hline${ }^{60} \mathrm{Co}$ Error & $7.00 \mathrm{E}-1$ & $5.50 \mathrm{E}+0$ & $2.30 \mathrm{E}+0$ & $1.30 \mathrm{E}+0$ \\
\hline${ }^{106} \mathrm{Ru}$ & $1.07 \mathrm{E}+6$ & $6.73 E+5$ & & $2.60 \mathrm{E}+5$ \\
\hline${ }^{106} \mathrm{Ru}$ Error & $4.06 \mathrm{E}+1$ & $3.00 \mathrm{E}+0$ & & $9.30 \mathrm{E}+0$ \\
\hline${ }^{125} \mathrm{Sb}$ & $6.26 \mathrm{E}+4$ & $2.28 \mathrm{E}+4$ & $2.68 \mathrm{E}+3$ & $7.06 \mathrm{E}+4$ \\
\hline${ }^{125} \mathrm{Sb}$ Error & $2.20 \mathrm{E}+0$ & $1.60 \mathrm{E}+0$ & $4.40 \mathrm{E}+0$ & $8.00 \mathrm{E}-1$ \\
\hline${ }^{134} \mathrm{Cs}$ & $4.22 E+4$ & $2.27 \mathrm{E}+2$ & $2.35 \mathrm{E}+2$ & $6.33 \mathrm{E}+3$ \\
\hline${ }^{134} \mathrm{Cs}$ Error & $3.90 E+0$ & $2.73 \mathrm{E}+1$ & $1.77 \mathrm{E}+1$ & $3.40 \mathrm{E}+0$ \\
\hline${ }^{137} \mathrm{Cs}$ & $2.49 \mathrm{E}+4$ & $1.18 \mathrm{E}+5$ & $690 \mathrm{E}+3$ & $2.84 \mathrm{E}+4$ \\
\hline${ }^{137} \mathrm{Cs}$ Error & $1.00 \mathrm{E}+0$ & $700 \mathrm{E}-1$ & $9.00 \mathrm{E}-1$ & $800 \mathrm{E}-1$ \\
\hline${ }^{144} \mathrm{Ce}$ & & & & $1.29 \mathrm{E}+7$ \\
\hline${ }^{144} \mathrm{Ce}$ Error & & & & $1.10 \mathrm{E}+0$ \\
\hline${ }^{152} \mathrm{Eu}$ & $1.70 \mathrm{E}+3$ & & $6.94 \mathrm{E}+1$ & $8.34 \mathrm{E}+3$ \\
\hline${ }^{152}$ Eu Error & $3.00 \mathrm{E}+0$ & & $1.09 \mathrm{E}+1$ & $9.00 \mathrm{E}-1$ \\
\hline${ }^{154} \mathrm{Eu}$ & $2.95 \mathrm{E}+3$ & & $8.77 \mathrm{E}+1$ & $1.98 \mathrm{E}+4$ \\
\hline${ }^{154}$ Eu Error & $2.20 \mathrm{E}+0$ & & $117 E+4$ & $7.00 \mathrm{E}-1$ \\
\hline${ }^{155} \mathrm{Eu}$ & $4.94 \mathrm{E}+4$ & & $2.93 E+2$ & $5.11 \mathrm{E}+4$ \\
\hline${ }^{155}$ Eu Error & $1.00 \mathrm{E}+0$ & & $8.40 \mathrm{E}+0$ & $1.00 \mathrm{E}+0$ \\
\hline${ }^{241} \mathrm{Am}$ & $3.04 E+3$ & & $787 \mathrm{E}+1$ & \\
\hline${ }^{241} \mathrm{Am}$ Error & $6.00 \mathrm{E}+0$ & & $1.91 \mathrm{E}+1$ & \\
\hline
\end{tabular}

\section{POST-LEACHING RESULTS}

\section{Radiochemistry and Elemental Analysis of Leaching Solutions}

The leaching solutions were separated from the solid phase and analyzed for radionuclides dissolved in solution as well as changes in major cations and $\mathrm{pH}$ relative to the composition of the pre-leaching solution. Leaching experiments were terminated at the end of 8 days, 31 days, 156 days, and 391 days and results from each are reported separately. Radiochemical results are listed in Table VI and reported in DPM / $\mathrm{ml}$ corrected to event 
zero-time. Changes in major cation concentrations and $\mathrm{pH}$ of the leaching solution relative to the starting composition of the Water Well 4A groundwater are displayed in Figure 1.

8-day Leaching Trial (\#1)

The 8-day leaching trial indicates the presence of ${ }^{22} \mathrm{Na},{ }^{60} \mathrm{Co},{ }^{125} \mathrm{Sb}$, and ${ }^{137} \mathrm{Cs}$ in the core $\# 1$, \#2, and \#8 solutions (identified hereafter as the \#1, \#2, and \#8 solutions). No radioactivity was detected in the \#6 solution. The \#2 and \#8 solutions carry both ${ }^{125} \mathrm{Sb}$ and ${ }^{137} \mathrm{Cs} ;{ }^{22} \mathrm{Na},{ }^{60} \mathrm{Co}$, and ${ }^{137} \mathrm{Cs}$ are detected only in solution \#1. Relative to the composition of the pre-leaching solution, all 8-day solutions show an enrichment in the alkalis and a depletion in Si. In particular, the \#2 and \#6 solutions show an almost $200 \%$ enrichment in $\mathrm{K}$. The \#6 solution is depleted in $\mathrm{Ca}$ and strongly enriched in $\mathrm{Mg}$.

31-day Leaching Trial (\#2)

Again ${ }^{22} \mathrm{Na},{ }^{125} \mathrm{Sb}$, and ${ }^{137} \mathrm{Cs}$ are detected only in the \#1, \#2, and \#8 solutions. ${ }^{125} \mathrm{Sb}$ and ${ }^{137} \mathrm{Cs}$ occur together in the \#2 solution. ${ }^{22} \mathrm{Na}$ and ${ }^{125} \mathrm{Sb}$ are detected in the \#1 and \#8 solutions respectively. As before, the major element chemistry indicates enrichments in the alkalis; the \#2 and \#6 solutions show similar high concentrations of $\mathrm{K}$. The \#6 solution is again depleted in $\mathrm{Ca}$ and enriched in $\mathrm{Mg}$.

156-day Leaching Trial (\#3)

The radiochemical results from the 156 trial are similar to the shorter trials. ${ }^{125} \mathrm{Sb}$ and ${ }^{137} \mathrm{Cs}$ appear together in the \#6 and \#8 solutions. ${ }^{22} \mathrm{Na}$ occurs in both the \#1 and \#6 solutions while the \#8 solution contains ${ }^{125} \mathrm{Sb}$ only. The major clement composition of the 156-day trial solution is identical to the results for the 31-day trial.

The 391-day Leaching Trial (\#4)

Like the shorter trials, ${ }^{22} \mathrm{Na},{ }^{125} \mathrm{Sb}$, and ${ }^{137} \mathrm{Cs}$ are the only radionuclides detected in the 391 day trial solutions. ${ }^{125} \mathrm{Sb}$ is dissolved in all the samples; in the \#1, \#2, and \#8 solutions ${ }^{125} \mathrm{Sb}$ occurs together with ${ }^{137} \mathrm{Cs} .{ }^{22} \mathrm{Na}$ occurs only in the core \#1 solution. Like the other trials, changes in the major element composition of the 391-day solutions relative to preleach compositions indicates an strong enrichment in the alkalis. 
8 Day Leachate - Major Element Composition

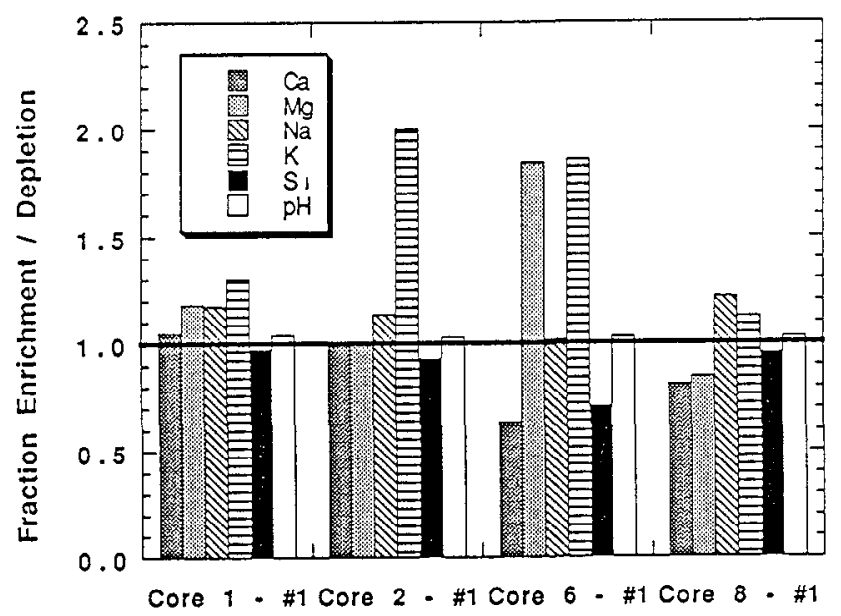

156 Day Leachate - Major Element Composition

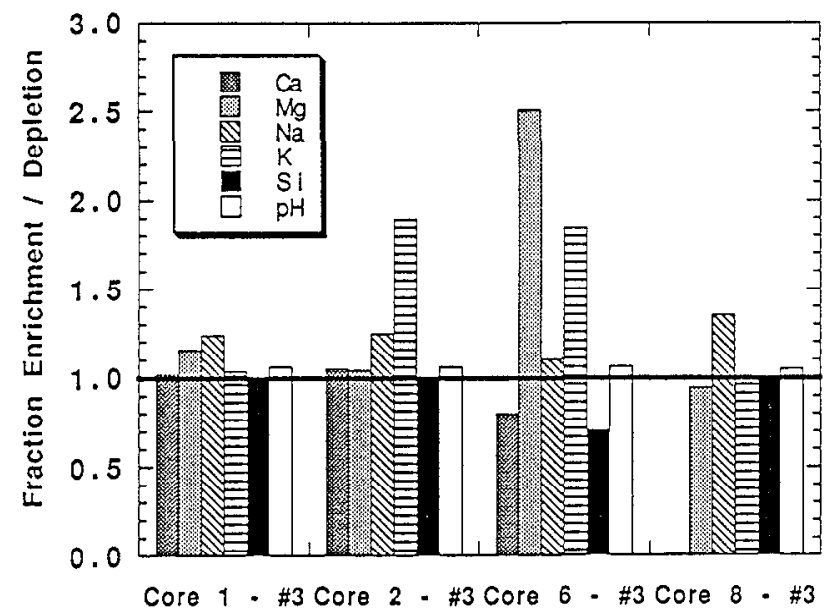

31 Day Leachate - Major Element Composition

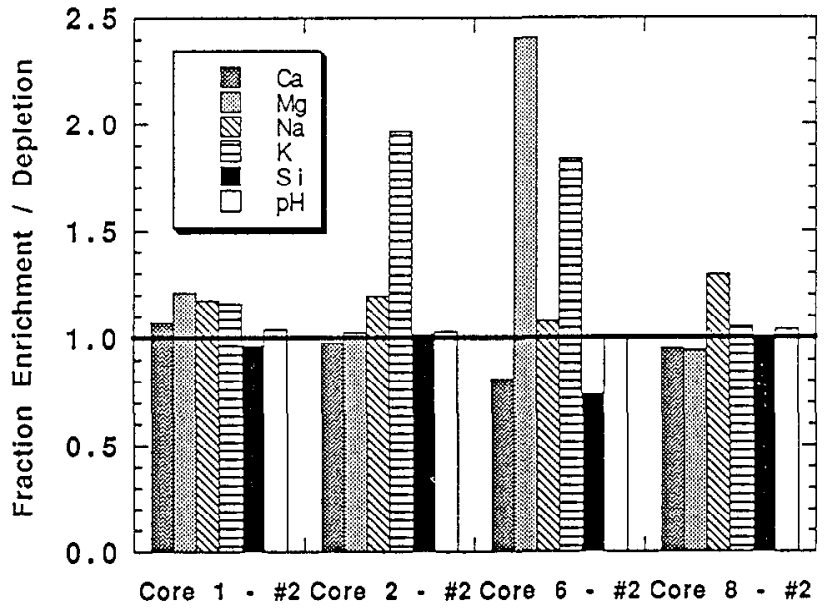

391 Day Leachate - Major Element Composition

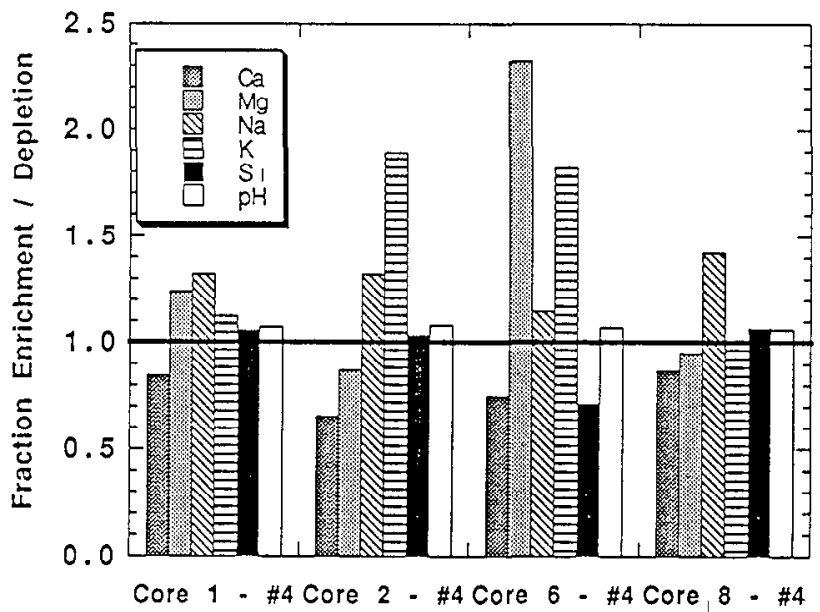

Figure 1: Changes in major element chemistry relative to starting composition of leaching solution (black line) for four nuclear explosive melt debris matrices. Core \#1 and core \#8 are dominated by glass; core \#2 and core \#8 are crystalline breccias. 
TABLE VI

Gamma Analyses of Core Leaching Solutions in DPM / ml Corrected to Event Zero-time

\begin{tabular}{|c|c|c|c|c|c|c|c|c|}
\hline & ${ }^{22} \mathrm{Na}$ & $\begin{array}{l}{ }^{22} \mathrm{Na} \\
\text { Error }\end{array}$ & ${ }^{60} \mathrm{Co}$ & $\begin{array}{l}{ }^{60} \mathrm{Co} \\
\text { Error }\end{array}$ & ${ }^{125} \mathrm{Sb}$ & $\begin{array}{l}{ }^{125} \mathrm{Sb} \\
\text { Error }\end{array}$ & ${ }^{137} \mathrm{Cs}$ & $\begin{array}{l}{ }^{137} \mathrm{Cs} \\
\text { Error }\end{array}$ \\
\hline Core 1 -\#1 & $1.01 E+2$ & $8.80 \mathrm{E}+0$ & $1.07 \mathrm{E}+1$ & $1.73 \mathrm{E}+1$ & & & $2.89 \mathrm{E}+0$ & $3.13 \mathrm{E}+1$ \\
\hline Core 2-\#1 & & & & & $1.02 \mathrm{E}+2$ & $7.20 \mathrm{E}+0$ & $8.47 \mathrm{E}+1$ & $1.90 \mathrm{E}+0$ \\
\hline \multicolumn{9}{|l|}{ Core 6 -\#1 } \\
\hline Core 8-\#1 & & & & & $2.84 \mathrm{E}+1$ & $2.06 \mathrm{E}+1$ & $2.19 E+0$ & $2.68 \mathrm{E}+1$ \\
\hline Core 1-\#2 & $1.81 \mathrm{E}+2$ & $1.29 \mathrm{E}+1$ & & & & & & \\
\hline Core 2-\#2 & & & & & $1.34 \mathrm{E}+2$ & $2.60 \mathrm{E}+1$ & $3.62 \mathrm{E}+2$ & $2.10 \mathrm{E}+0$ \\
\hline \multicolumn{9}{|l|}{ Core 6-\#2 } \\
\hline Core 8-\#2 & & & & & $5.01 \mathrm{E}+1$ & $2.67 \mathrm{E}+1$ & & \\
\hline Core 1-\#3 & $2.10 \mathrm{E}+2$ & $1.32 \mathrm{E}+1$ & & & & & & \\
\hline Core $2-\# 3$ & & & & & $1.82 E+2$ & $1.25 \mathrm{E}+1$ & $9.18 \mathrm{E}+1$ & $3.10 \mathrm{E}+0$ \\
\hline Core 6-\#3 & $7.35 \mathrm{E}+0$ & $4.46 \mathrm{E}+1$ & & & $3.18 \mathrm{E}+1$ & $2.12 \mathrm{E}+1$ & $9.64 \mathrm{E}-1$ & $3.71 \mathrm{E}+1$ \\
\hline Core $8-\# 3$ & & & & & $6.69 \mathrm{E}+1$ & $2.81 \mathrm{E}+1$ & & \\
\hline Core $1-\# 4$ & $1.61 \mathrm{E}+2$ & $1.60 \mathrm{E}+1$ & & & $4.87 \mathrm{E}+1$ & $2.80 \mathrm{E}+1$ & $1.16 \mathrm{E}+0$ & $2.70 \mathrm{E}+1$ \\
\hline Core 2-\#4 & & & & & $1.74 \mathrm{E}+2$ & $2.10 \mathrm{E}+1$ & $5.68 \mathrm{E}+1$ & $4.30 \mathrm{E}+0$ \\
\hline Core 6-\#4 & & & & & $2.58 \mathrm{E}+1$ & $2.00 \mathrm{E}+1$ & & \\
\hline Core 8 -\#4 & & & & & $3.29 E+1$ & $1.20 \mathrm{E}+1$ & $1.26 \mathrm{E}+0$ & $2.10 \mathrm{E}+1$ \\
\hline
\end{tabular}

\section{Electron Imaging of Leached Solids}

Leached solids from each of the four trials were dried at $25^{\circ} \mathrm{C}$, potted in epoxy, sectioned with a wafering saw and finished to a high polish. In this way, a cross sectional area of the grains were exposed which reveled surface reactions affecting single grains. These crosssections were imaged with back-scattered electrons using an electron microscope. Representative images are displayed in Figure 2.

The glass samples from core \#1 and \#8 revealed sharp conchoidal fracture surfaces with little or no surface alteration. Individual grain boundaries were distinct; gel layers and following secondary minerals characteristic of the alteration of high-level nuclear waste borosilicate glasses $\left(\sim 50\right.$ weight $\left.\% \mathrm{SiO}_{2}\right)$ were not observed (i.e., Abrajano et al., 1990). The crystalline mineral samples of core \#2 and \#6 exhibit an alteration layer $\sim 5 \mu \mathrm{m}$ or more in thickness. The alteration layer is variably developed on individual grain surfaces and has an irregular thickness. In many cases the alteration layer completely surrounds an otherwise homogeneous grain. In some grains, the alteration layer appears as if it is partially detached from the interior, unreacted matrix. A conspicuous alteration layer is only associated with the leached mineral grains; the alteration layer is not observed on the glassy samples. 

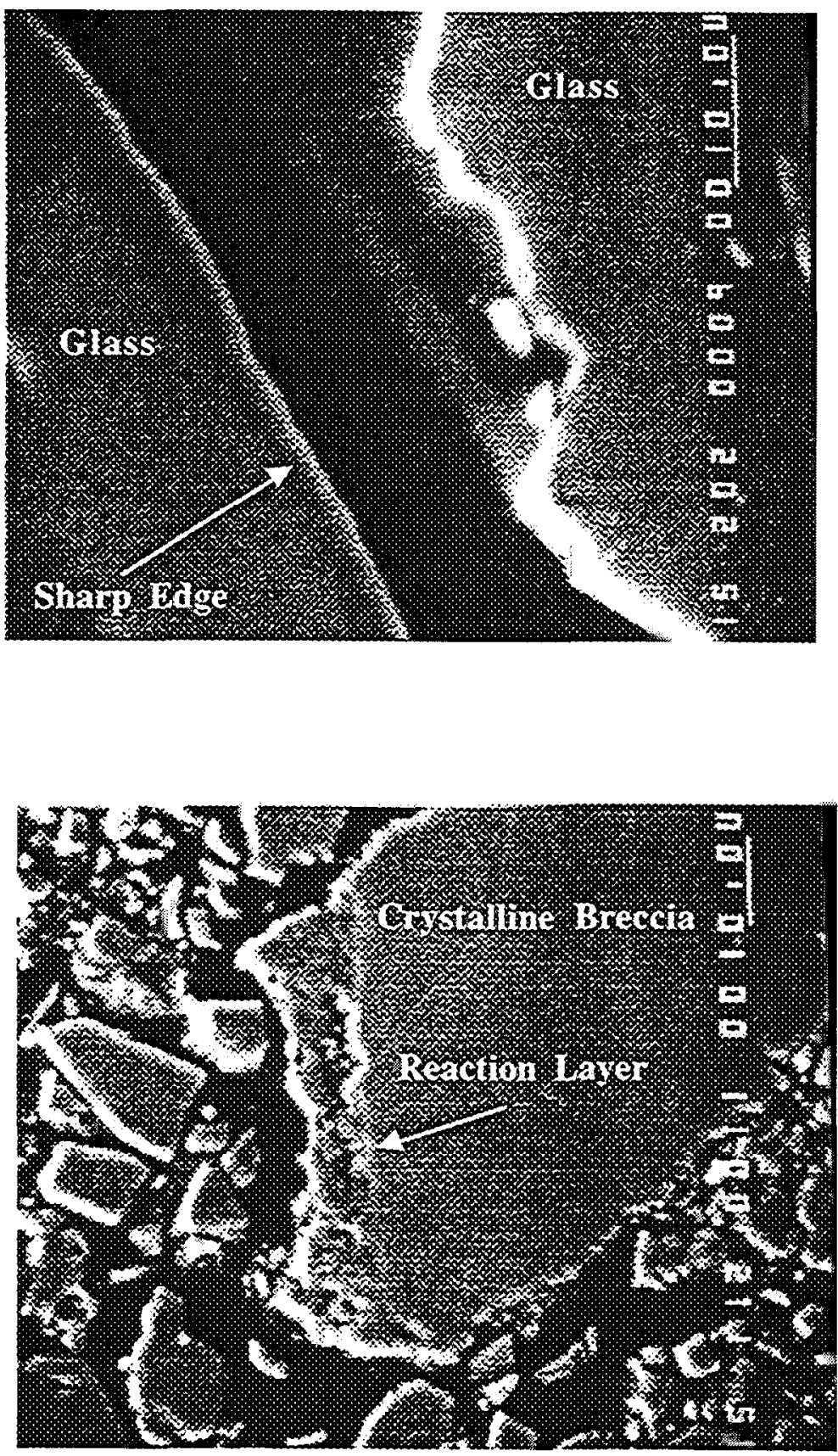

Figure 2: Backscattered clectron images of the cross-section areas of grains leached for 31 days. Glass sample above, crystalline breccia sample below. $10 \mu \mathrm{m}$ scale bar. Note extensive surface reaction involving brccia sample while sharp edge of glass sample exhibits little reaction. 


\section{INTERPRETATION}

The results of static leaching experiments of nuclear explosive melt debris indicate that the initial hydrologic source term is composed of volatile and soluble radionuclides leached from the crystalline matrices. The leaching experiments indicate only slight dissolution of the melt glass.

Pre-leach characterization of the nuclear explosive melt debris taken from four different nuclear explosions indicates that both glassy and crystalline matrices incorporate a variety of radionuclides. Glassy samples include both volatile and refractory species. The refractory radionuclides are most strongly enriched in the glass that coalesces on the floor of the standing cavity at relatively high temperatures. Volatile radionuclides are more widely dispersed and are found associated with the quartz and feldspar rich samples that are representative of samples taken at the bottom of the collapse chimney where rubble is mixed in the glass puddle at the bottom of the cavity. The matrix consists of mineral constituents including quartz and feldspar - anticipated from the working point geology - as well as calcite and dolomite which are sediments incorporated in the alluvium of the Yucca Flat testing area. It is important to note that radioactivity residual from an underground nuclear explosion is associated not only with conventional black "melt glass" but is also associated with crystalline materials that represent regions outside of the immediate explosion cavity. The crystalline material contains volatiles that condense on fracture and grain surfaces at lower temperature during and following collapse of the standing cavity. Crystalline debris in the present study contains ${ }^{22} \mathrm{Na},{ }^{106} \mathrm{Ru},{ }^{125} \mathrm{Sb}$, and ${ }^{134} \mathrm{Cs}$ and is enriched in ${ }^{137} \mathrm{Cs}$ which has a extremely diffusive ${ }^{137} \mathrm{Xe}$ noble gas precursor. Crystalline materials occur intermixed with the puddle region and within $\sim 10 \mathrm{~m}$ from the measured bottom of the puddle. The presence of volatile radionuclides in the chimney and collapse zone represents an important source of radionuclides that are readily avilable for dissolution in groundwater. The presence of volatile radionuclides in the chimney region was previously reported by Thompson (1997) and Smith et al., (1997).

The results of the leaching experiments suggest that only the most soluble radionuclides incorporated in the glass actually dissolve in the dilute bicarbonate groundwaters under static leaching conditions. The composition of the melt debris and the leachate controls the susceptibility of radionuclide dissolution. Ion exchange and dissolution reactions which occur at mineral and grain surfaces likely release radionuclides to solution. Imaging analysis of mineral and grain surfaces following each of the four leaching trials indicates that the minerals and crystalline matrices exhibited strong evidence of surface reaction. Strong alkali enrichment and exchange of the alkaline earths in the leachate chemistry further confirms this conclusion. 


\section{Equilibrium Modeling}

To better evaluate the controls on the dissolution of the melt debris, the geochemistry of the leaching solution was modeled to determine what minerals are in equilibrium with the Water Well 4A leaching solution. Calculations were run using React which is part of the Geochemist's Workbench (Bethke, 1994). Knowledge of the equilibrium state of the reacting fluid provides insight to the susceptibility of minerals and glass in the melt debris to dissolve in dilute bicarbonate groundwater. Modeling results are presented in Table VII ranked by saturation index $(\log (\mathrm{Q} / \mathrm{K})$, high to low), where $\mathrm{Q}$ is the activity product and $\mathrm{K}$ is the equilibrium constant.

TABLE VII

Equilbrium Modeling Results of Water Well 4A Groundwater Relative to Saturation Index of Selected Minerals (in rank order)

\begin{tabular}{ll}
\hline Saturated Minerals & Unsaturated Minerals \\
\hline & \\
Nontronite, $\mathrm{Ca}, \mathrm{Mg}, \mathrm{Na}, \mathrm{K}, \mathrm{H}$ & Calcite \\
Clinoptilolite & $\mathrm{SiO} 2(\mathrm{am})$ \\
Hematite & Aragonite \\
Montmorillonite, $\mathrm{Mg}, \mathrm{Ca}, \mathrm{K}$ & Ferrite, $\mathrm{Mg}, \mathrm{Ca}$ \\
Illite & Natrolite \\
Beidellite, $\mathrm{Ca}, \mathrm{Mg}, \mathrm{Na}, \mathrm{K}$ & Clinochlore \\
Kaolinite & Magnesite \\
K-feldspar & $\mathrm{Fe}(\mathrm{OH}) 3$ \\
Mordenite & Corundum \\
Saponite, $\mathrm{Ca}, \mathrm{Mg}, \mathrm{Na}, \mathrm{K}, \mathrm{H}$ & Sepolite \\
Talc & Kyanite \\
Albite & Andalusite \\
Quartz & Fluorite \\
Tridymite & Gypsum \\
Dolomite & Anhydrite \\
Chalcedony & Tremolite \\
\hline
\end{tabular}

Equilibrium modeling suggests that at near-neutral $\mathrm{pH}, \mathrm{HCO}_{3}, \mathrm{Na}, \mathrm{Ca}, \mathrm{SO}_{4}, \mathrm{Cl}, \mathrm{K}$, and $\mathrm{CO}_{2}$ (aq) occur as dominant free ions; the most concentrated molecular species are $\mathrm{SiO}_{2}(\mathrm{aq})$, $\mathrm{CaSO}_{4}, \mathrm{MgSO}_{4}, \mathrm{CaHCO}_{3}$, and $\mathrm{O}_{2}(\mathrm{aq})$. The reacting fluid is saturated with respect to quartz, feldspars, clays, zeolites, and carbonate. The silica saturation of the leaching solution attenuates bulk matrix reaction of the crystalline or glass samples. These results explain the near constancy of $\mathrm{Si}$ in the leaching solution relative to its pre-leach composition. The glass samples show little release of the alkalis or alkaline earths. That only minimal reaction occurs is verificd both from radiochemical analyses of the leachate which indicate very low concentrations of radionuclides in solution as well as imaging results which show a sharp glass grain surface without a developed reaction layer. For the crystalline samples, the exchange of alkalis into solution is notable; the enrichment of $\mathrm{K}$ 
over $\mathrm{Na}$ suggests that $\mathrm{K}$-feldspar may preferentially dissolve and release $\mathrm{K}$ to solution. $\mathrm{A}$ dissolution reaction might be:

$$
\begin{gathered}
\mathrm{KAlSi}_{3} \mathrm{O}_{8}+8 \mathrm{H}_{2} \mathrm{O}=\mathrm{K}^{+}+\mathrm{Al}(\mathrm{OH})_{4}^{-}+3 \mathrm{H}_{4} \mathrm{SiO}_{4} \\
2 \mathrm{KAlSi}_{3} \mathrm{O}_{8}+4 \mathrm{Al}(\mathrm{OH})_{3}+\mathrm{H}_{2} \mathrm{O}=3 \mathrm{Al}_{2} \mathrm{Si}_{2} \mathrm{O}_{5}(\mathrm{OH})_{4}+2 \mathrm{~K}^{+}+2 \mathrm{OH}^{-}
\end{gathered}
$$

This reaction produces kaolinite from the alteration of K-feldspar. Ion exchange may also affect surface reaction, particularly in the case of the \#6 solution which is associated with the carbonate matrix. In this case, $\mathrm{Ca}$ is depleted and $\mathrm{Mg}$ is enriched in the leaching solution. After dissolution of calcite, Ca may be exchanged with $\mathrm{Mg}$ particularly in the presence of smectite clays (i.e., montmorillonite: $\left.(\mathrm{Na}, \mathrm{Ca})_{03}(\mathrm{Al}, \mathrm{Mg})_{2} \mathrm{Si}_{4} \mathrm{O}_{10}(\mathrm{OH})_{2} \bullet \mathrm{nH}_{2} \mathrm{O}\right)$.

\section{Calculation of Dissolution Rate}

Failor et al. (1983) and Coles et al. (1978) calculated the dissolution rate for melt-glass samples using the following equation:

$$
\text { leach rate }=R=\left(A / A_{0}\right)(1 / \Delta t)(1 / S)
$$

where:

$\mathrm{R}=$ fraction leached per day per $\mathrm{m}^{2}$ surface area ( $\mathrm{g}$-solid $/ \mathrm{m}^{2} /$ day)

$\mathrm{A}=$ total activity leached during $\Delta \mathrm{t}$

$A_{o}=$ pre-leach specific activity in melt-debris sample per gram

$\Delta \mathrm{t}=$ interval of leaching, days

$\mathrm{S}=$ specific surface area of the solid in $\mathrm{m}^{2} / \mathrm{g}$-solid 
Table VIII lists dissolution rates in grams $/ \mathrm{m}^{2} /$ day:

TABLE VIII

Dissolution Rates Calculated for Nuclear Explosive Melt Debris Relative to $8,31,156$, and 391 Day Leaching Trials (in grams $/ \mathrm{m}^{2} /$ day)

\begin{tabular}{llll}
\hline & ${ }^{22} \mathrm{Na}$ & ${ }^{125} \mathrm{Sb}$ & ${ }^{137} \mathrm{Cs}$ \\
\hline Core 1 - \#1 & $2.98 \mathrm{E}-1$ & - & $4.02 \mathrm{E}-3$ \\
Core 2 - \#1 & - & $1.03 \mathrm{E}-1$ & $1.64 \mathrm{E}-2$ \\
Core 6 - \#1 & - & - & - \\
Core 8 -\#1 & - & $1.15 \mathrm{E}-2$ & $2.22 \mathrm{E}-3$ \\
Core 1 - \#2 & $1.37 \mathrm{E}-1$ & - & - \\
Core 2 - \#2 & - & $3.48 \mathrm{E}-2$ & $1.81 \mathrm{E}-2$ \\
Core 6 - \#2 & - & - & - \\
Core 8 - \#2 & - & $5.26 \mathrm{E}-3$ & - \\
Core 1 - \#3 & $3.18 \mathrm{E}-2$ & - & - \\
Core 2 - \#3 & - & $9.38 \mathrm{E}-3$ & $9.14 \mathrm{E}-4$ \\
Core 6 - \#3 & $7.02 \mathrm{E}-2$ & $1.39 \mathrm{E}-2$ & $1.64 \mathrm{E}-4$ \\
Core 8 - \#3 & - & $1.40 \mathrm{E}-3$ & - \\
Core 1 - \#4 & $9.70 \mathrm{E}-3$ & $5.50 \mathrm{E}-4$ & $3.30 \mathrm{E}-5$ \\
Core 2 - \#4 & - & $3.58 \mathrm{E}-3$ & $2.26 \mathrm{E}-4$ \\
Core 6 - \#4 & - & $4.52 \mathrm{E}-3$ & - \\
Core 8 - \#4 & - & $2.74 \mathrm{E}-4$ & $2.60 \mathrm{E}-5$ \\
\hline
\end{tabular}

The leach rates determined here compare favorably with those determined in by earlier flow-through experiments (Coles et al., 1978; Failor et al., 1983). Leaching rates are fastest for ${ }^{22} \mathrm{Na}$, followed by ${ }^{125} \mathrm{Sb}$, and slowest for ${ }^{137} \mathrm{Cs}$. Generally, leaching rates for the crystalline solids (core \#2 and core \#6) are greater than those for the glass (core \#1 and core \#8). The magnitude of the leaching rates are inverscly proportional to the length of the experiment; the inventory of volatile radionuclides which condense on fracture and mineral surfaces is likely depleted after initial exchange. Variations in absolute leach rate between expcriments may be duc to 1) differences in the method used to determine specific surface area between earlier studies and this study, 2) differences between batch leaching used in this study and flow-through studies used by Coles et al. (1978) and Failor et al. (1983), and 3) analytical error (the gamma counting error for individual radionuclides in solution is generally 10 to $20 \%$ ).

\section{CONCLUSIONS}

The release of soluble radionuclides to groundwater which condense on mineral and rock surfaces in the region affected by inelastic fracturing away from the working point defines the initial hydrologic source term. Radionuclides residual from nuclear testing are associated with both glassy and crystalline matrices. Volatile radionuclides (e.g., Na, Sb, $\mathrm{Ru}, \mathrm{Cs})$ fractionate in the plasma created during the detonation and may be separated by 
incondensible gases (e.g., $\mathrm{CO}_{2}, \mathrm{H}_{2}$ ) and steam that fill the standing cavity. The volatile radionuclides condense at lower temperatures where they are sited on fracture and mineral surfaces associated with chimney collapse and subsequently become incorporated with the fused debris in the melt. Refractory radionuclides (e.g., $\mathrm{Zr}, \mathrm{Ce}, \mathrm{Eu}$ ) are exclusively associated with the melt glass that condenses at higher temperatures $\left(\sim 1000^{\circ} \mathrm{C}\right)$. The crystalline breccias include both quartz and feldspars (K, $\mathrm{Na}$, and $\mathrm{Ca})$ as well as calcite and dolomite derived from clasts in the alluvium of Yucca Flat.

One year leaching results indicate that only the most soluble radionuclides are transferred from the melt debris to solution. Alkalis are soluble in the dilute bicarbonate groundwaters of the Nevada Test Site. The mineral and major element composition of the melt debris and the composition of the leachate controls the susceptibility of radionuclides to be released to solution; radionuclides associated with surface coatings on crystalline matrices preferentially dissolve relative to those incorporated in the glass samples. Electron imaging indicates that dissolution may affect $\mathrm{a} \sim 5 \mu \mathrm{m}$ thick surface layer that is well developed on the crystalline matrices; the glass samples do not exhibit a well defined reaction layer. Equilibrium modeling suggests that lower dissolution rates are due to the saturation of silica and quartzo-feldspathic minerals in waters beneath Yucca Flat; this likely also explains why the glass samples show no textural evidence of reaction. Dissolution rates for the crystalline solids range betwecn $1.0 \mathrm{E}-1\left({ }^{125} \mathrm{Sb}\right)$ and $1.6 \mathrm{E}-4\left({ }^{137} \mathrm{Cs}\right) \mathrm{grams} / \mathrm{m}^{2} /$ day. Dissolution rates for glass samples range between $3.0 \mathrm{E}-1\left({ }^{22} \mathrm{Na}\right)$ and $2.6 \mathrm{E}-5\left({ }^{137} \mathrm{Cs}\right)$ $\mathrm{grams} / \mathrm{m}^{2} /$ day. For the same radionuclide, dissolution rates are faster for the crystalline matrices than the glasses.

These experiments suggest that volatile radionuclides (and shorter half-lives: $<\sim 30$ years) associated with surface reactions contribute to an initial hydrologic source term. Refractory radionuclides, although partitioned in the glass, apparently do not comprise hydrologic source term at least initially. Dissolution rates compare favorably with rates determined by earlier flow-through experiments using nuclear explosive melt glass samples. Results of static leaching experiments are meant to be illustrative of the controls on the release of radionuclides to solution. It should be noted that not all radionuclides are released from the cavity region by dissolution; transport of radionuclides adjacent to nuclear tests may also occur by non-solute process including prompt injection (Nimz and Thompson, 1992), gaseous transport (Smith et al., 1996), and colloidal movement (Buddemeier and Hunt, 1988; Thompson, 1998). As well, a majority of radionuclides have decayed during the approximate ten year interval between recovery of the debris and the present study. Nevertheless, results of this experiment suggest that under static conditions - expected in regions of lower groundwater flow - the release of soluble radionuclides will occur by ion exchange and mild dissolution reactions. As such, solution of radionuclides in groundwater does not necessarily involve a point source release from the dissolution of 
glass in the puddle region but rather release from mineral and fracture surfaces throughout the saturated cavity-chimney region which may extended out to a distance of $\sim 2$ cavity radii or more from the working point.

\section{ACKNOWLEDGEMENTS}

These experiments could not have been completed without the expertise of Ruth Nuckolls of the Chemistry and Materials Science (C\&MS) gamma counting facility and Terry Duewer of the C\&MS ICP-AES laboratory. Jackie Kenneally collected the Water Well 4A leaching solution at the Nevada Test Site. Bill Bourcier of Geosciences and Environmental Technology provided a helpful review of this work.

\section{REFERENCES}

Abrajano, T.A., J.K. Bates, A.B. Woodland, J.P. Bradley, and W.L. Bourcier, 1990, Clays and Clay Minerals, v. 38, p. 537-548.

Bethke, C., 1994, The Geochemist's Workbench, version 2.0, a users guide to Rxn, Act2, Tact, React, and Gtplot, Hydrogeology Program, University of Illinois, 213p.

Borg, I.Y., 1975, Radioactivity trapped in melt produced by a nuclear explosion, Nuclear Technology, v. 26, p. 88-100.

Borg, I.Y., R. Stone, H.B. Levy, and L.D. Ramspott, 1976, Information pertinent to the migration of radionuclides in ground water at the Nevada Test Site, Part 1: revicw of existing information, Lawrence Livermore National Laboratory, UCRL-52078 Pt.1, 216p.

Bourcier, W.L., 1990, Geochemical modeling of radioactive waste glass dissolution using EQ3/6: preliminary results and data needs, Lawrence Livermore National Laboratory, UCID-21869, 37p.

Bourcier, W.L., 1994, Critical review of glass performance modeling, Argonne National Laboratory, ANL-94/17, 64p.

Buddemeier, R.W. and J.R. Hunt, 1988, Transport of colloidal contaminants in groundwater: radionuclide migration at the Nevada Test Site, Applied Geochemistry, v. 3, p. $535-548$.

Coles, D.G, H.C. Weed, D.D. Jackson, J.S. Schweiger, 1978, Single-pass leaching of nuclear melt glass by groundwater, part I. Lawrence Livermore Laboratory, UCRL-81617, Preprint, 32p.

Esser, B.K., 1995, Sample characterization of nuclear explosive melt debris for initial radionuclide distribution, unpublished letter report to U.S. Department of Energy, Nevada Operations Office, Environmental Restoration Division, 10p. 
Essington, E.H., and J.V.A. Sharp, 1968, Some aspects of ground-water solution chemistry, underground nuclear explosion zones, Nevada Test Site, in E.B. Eckel (ed.) Nevada Test Site, Geological Society of America, Memoir 110, p. 263- 273.

Failor, R.A., D.G. Coles, J.H. Rego, 1983, A leaching study of nuclear melt glass: part I, Lawrence Livermore National Laboratory, UCID-19729, 71p.

Kersting, A.B., 1996, The state of the hydrologic source term, Lawrence Livermore National Laboratory, UCRL-ID-126557, 30p.

Nimz, G.J. and J.L. Thompson, 1992, Underground radionuclide migration at the Nevada Test Site, U.S. Department of Energy, Nevada Field Office, DOE/NV-346, 17p.

Schwartz, L., A. Piwinskii, F. Ryerson, H. Tewes, and W. Beiriger, 1984, Glass produced by underground nuclear explosions, in L.D. Pye, J. A. O'Keefe, and V.D. Frechette, eds., Natural Glasses, North-Holland, p. 559-591.

Smith, D.K., 1995, Characterization of nuclear explosive melt debris, Radiochimica Acta, v. 69, p. $157-167$.

Smith, D.K., R.J. Nagle, and J.M. Kenneally, 1996, Transport of gaseous fission products adjacent to an underground nuclear test cavity, Radiochimica Acta, v. 73, p. 177183.

Smith, D.K., 1993, A review of literature pertaining to the leaching and sorption of radionuclides associated with nuclear explosive melt glasses, Lawrence Livermore National Laboratory, UCRL-ID-113370, 26p.

Smith, D.K., A.B. Kersting, J.M. Kenneally, J.H. Rego, and J.L. Thompson, 1997, Hydrologic Resources Management Program FY 1996 Progress Report, Lawrence Livermore National Laboratory, UCRL-ID-126886, 40p.

Thompson, J.L. 1990, Laboratory and field studies related to the Hydrology/Radionuclide Migration Project, Los Alamos National I aboratory, LA-11947-PR, 21p.

Thompson, J.L., 1991, Laboratory and field studies related to the Hydrology/Radionuclide Migration Project, Los Alamos National Laboratory, LA-12100-PR, 38p.

Thompson, J.L., 1994, Laboratory and field studies related to the Hydrologic Resources Management Program, Los Alamos National Laboratory, LA-12721-PR, $31 \mathrm{p}$.

Thompson, J.L., 1996, Radionuclide distribution in a nuclear test cavity: the Baseball event, Radiochimica Acta, v. 72, p. $157-162$.

Thompson, J.L., 1997, Laboratory and field studies related to the Hydrologic Resources Management Program, Los Alamos National Laboratory, LA-13270-PR, 24p.

Thompson, J.L., 1998, Laboratory and field studies related to radionuclide migration at the Nevada Test Site, Los Alamos National Laboratory, LA-13419-PR, 31p. 


\section{CHAPTER 6}

\section{ISOTOPE HYDROLOGY INVESTIGATION OF REGIONAL GROUNDWATER FLOW IN CENTRAL NEVADA}

Timothy P. Rose, M. Lee Davisson, David K. Smith and Jacqueline M. Kenneally

\section{SUMMARY}

New stable isotope and radiocarbon data are presented for surface and groundwater samples from central Nevada. These data are combined with previous LLNL data to create a $\delta^{18} \mathrm{O}$ contour map of regional groundwater flow patterns in central and southern Nevada. The results suggest that a continuous flow path exists between the Railroad Valley in central Nevada and Pahute Mesa in the northwestern Nevada Test Site. Groundwater is transported southward more than $200 \mathrm{~km}$ through this system along a series of interconnected basins. Groundwater with $\delta^{18} \mathrm{O}$ values in the range of -15.5 to $-16 \%$ originates in high elevation recharge areas in the upper Railroad Valley flow system. As the groundwater moves southward, it gradually mixes with relatively ${ }^{18} \mathrm{O}$ enriched recharge at lower elevations. Deep groundwaters beneath Pahute Mesa have stable isotope values that cannot be derived from local precipitation, but are readily cxplaincd by regional groundwater influx from the Railroad Valley flow system. Railroad Valley groundwaters show a rapid but systematic decrease in ${ }^{14} \mathrm{C}$ values between alpine recharge areas and the basin carbonate aquifer. $\delta^{13} \mathrm{C}$ values increase along the same flow path, reflecting chemical reaction with the carbonate host rock. Pahute Mesa groundwaters have ${ }^{14} \mathrm{C}$ and $\delta^{13} \mathrm{C}$ values that generally overlap with the Railroad Valley data. The Pahute Mesa data are consistent with mixing of Railroad Valley groundwater with local recharge and subsurface influx from adjacent basins in the lower part of the flow system.

\section{INTRODUCTION}

Understanding the transport rate of radionuclides from underground nuclear test cavities at the Nevada Test Site (NTS) requires an understanding of the regional and local hydrogeology. The recent discovery of colloidal transport of plutonium in groundwater at the NTS (Kersting et al., 1998) has underscored the need for a more comprehensive knowledge of groundwater flow patterns in this region. Although flow models have been created to evaluate regional groundwater transport through the NTS, these models are ultimately limited by the extent of the regional databases on which they are based. Geochemical studies using environmental isotope tracers provide direct evidence for the origin and transport rate of groundwater that is often complimentary to conventional hydrogeologic investigations. 
In this study, we have used environmental isotope tracers in a reconnaissance investigation of regional groundwater flow systems entering the Nevada Test Site from the north. The results of this study have important implications for the nature of regional groundwater transport through the Pahute Mesa region, and emphasize the need to consider the integrated nature of these flow systems.

\section{BACKGROUND}

The regional groundwater flow systems of the Great Basin have been the topic of numerous studies (cf. Winograd and Thordarson, 1975; Mifflin and Hess, 1979; Dettinger et al., 1995; Thomas et al., 1996). These interbasin flow systems are typified by fracturedominated flow through carbonate and volcanic rocks, driven by hydraulic gradients that are laterally continuous over hundreds of kilometers (Harrill et al., 1988). A well-known example is the carbonate-hosted White River flow system in castern Nevada (Maxcy and Eakin, 1949; Eakin, 1966).

Davisson et al. (1998) observed that regional groundwater flow systems in Nevada show systematic increases in $\delta^{18} \mathrm{O}$ values with decreasing latitude. For example, the White River system is characterized by a "plume" of low- ${ }^{18} \mathrm{O}$ water that extends southward along interconnected graben valleys in the direction of flow. Groundwater $\delta^{18} \mathrm{O}$ values gradually increase along this flow path due to mixing with higher $\delta^{18} \mathrm{O}$ recharge at lower latitudes (Davisson et al., 1998). However, at latitudes south of $\sim 38^{\circ} \mathrm{N}$, the amount of recharge from local sources is generally small, and the regional flow system exhibits isotopic values that are distinctly lower in $\delta^{18} \mathrm{O}$ and $\delta \mathrm{D}$ than local groundwater recharge.

Groundwater from the Pahute Mesa area of the NTS shows a similar discontinuity between the isotopic values of deep groundwater and local recharge. Most groundwaters from Pahute Mesa range in $\delta^{18} \mathrm{O}$ from -14.7 to $-15.1 \%$, with $\delta \mathrm{D}$ values between -110 and $-120 \%$ (Figure 1). Although Pahute Mesa snowfall has a weighted mean $\delta^{18} \mathrm{O}$ value similar to the groundwater $(-14.7 \%$ ) , it has a higher $\delta \mathrm{D}$ value $(-104 \%$ ) (data from Milne et al., 1987). Local rainfall is significantly higher in both $\delta^{18} \mathrm{O}$ and $\delta \mathrm{D}$ (see Figure 1). These data indicate that modern local precipitation cannot be the sole source of the groundwater.

Previous studies suggested that isotopically-depleted groundwaters such as those underlying Pahute Mesa were recharged locally under cooler climatic conditions that existed during the last glacial period (e.g. Claassen, 1986; Benson and Klieforth, 1989). However, this model is most compatible with closed-basin systems wherein groundwater recharge from pluvial periods is stored as a static mass that remains poorly mixed with modern recharge (e.g. Rose et al., 1997a). Groundwater flow beneath Pahute Mesa is 
considered to be a part of the Death Valley regional flow system, receiving recharge from the north in the Kawich and Reveille Ranges (see Figure 2; Blankennagel and Weir, 1973; Laczniak et al., 1996). It is noteworthy that spring water samples from the Kawich Range have stable isotope values that are too high in $\delta^{18} \mathrm{O}$ and $\delta \mathrm{D}$ to account for the Pahute Mesa groundwater (Figure 1). On the basis of environmental isotope data presented in the following report, we propose that the low- $\delta^{18} \mathrm{O}$, low- $\delta \mathrm{D}$ groundwater underlying Pahute Mesa originates from regional groundwater transport through the Railroad Valley flow system. This system encompasses a broad region that extends $>200$ $\mathrm{km}$ from Newark Valley in the north to Pahute Mesa in the south.

\section{HYDROGEOLOGIC SETTING}

Summaries of the hydrogeology of central and southern Nevada are found in Rush and Everett (1966), Blankennagel and Weir (1973), Van Denburgh and Rush (1974), Winograd and Thordarson (1975) and Laczniak et al. (1996). We will focus our attention on the Railroad Valley and Pahute Mesa regions. Three principal hydrostratigraphic units occur in this region: Paleozoic carbonate, Tertiary volcanic, and alluvial aquifers. Carbonate aquifers underlie alluvial and volcanic rock sequences in many areas of eastcentral and southern Nevada, and are probably the principal conduits for regional flow (Dettinger et al., 1995). Oil and gas exploration wells revealed the presence of carbonate bedrock beneath alluvial basin-fill deposits in the Railroad Valley (e.g. Hulen et al., 1994). Extensive outcrops of carbonate rock occur in the White Pine, Grant and Quinn Canyon Ranges, along the eastern boundary of the Railroad Valley, and in the Fish Creek and Hot Creek Ranges on the western side of the flow system (see Figure 2; Stewart and Carlson, 1978). Infiltration of snowmelt through fractured carbonate rock in these ranges is probably an important source of recharge to the carbonate rock aquifers.

Deep regional flow through carbonate rocks travels in a southerly direction from Newark Valley to the central Railroad Valley (Roth and Campana, 1989). Interbasin flow from the Little Smoky, Hot Creek and Big Sand Springs Valleys also contribute to the flow system (Rush and Everett, 1966; Van Denburgh and Rush, 1974; Harrill et al., 1988). The Railroad Valley flow system is generally considered to terminate in a large playa in the central Railroad Valley, although this interpretation is largely based on groundwater levels in wells that only perforatc ovcrlying basin-fill deposits (Bedinger et al., 1984; Thomas et al., 1986). Carbonate aquifer hydraulic head data does not exist for the southern Railroad Valley flow system, although geologic evidence suggests that carbonate rock may underlie basin-fill deposits as far south as Kawich Valley (Ekren et al., 1971). Groundwater is transported beneath Pahute Mesa in a south-southwest direction, through a thick sequence of fractured volcanic rock. Blankennagel and Weir 
(1973) estimated that approximately 5,500 acre-feet/year of groundwater enters Pahute Mesa from the north, principally from the Kawich Valley.

Estimated groundwater budgets for the Railroad Valley flow system are found in Rush and Everett (1966), Van Denburgh and Rush (1974), and Prudic et al. (1995). A useful summary is provided in Roth and Campana (1989), who estimate an annual hydrologic mass balance of approximately 115,000 acre-feet/year. In general, these models predict that recharge from precipitation and subsurface inflow is balanced by discharge from evapotranspiration and spring flow. It is notable that Van Denburgh and Rush (1974) estimated that only 4 percent of the annual precipitation budget recharges the Railroad Valley flow system. Uncertainties in these models far exceed the small volume of water $(5,500$ acre-feet/year) necessary to account for underflow from the Railroad Valley flow system to Pahute Mesa.

\section{SAMPLING AND ANALYTICAL METHODS}

A total of 33 water samples were collected from springs, creeks and wells in central Nevada, within an area approximately bounded by latitudes $38^{\circ} 00^{\prime}$ to $39^{\circ} 30^{\prime} \mathrm{N}$ and longitudes $115^{\circ} 30^{\prime}$ to $117^{\circ} 30^{\prime} \mathrm{W}$. All but one of the samples was collected in May 1997 . Sample locations, field and analytical data are reported in Table 1. Figure 2 is a map of central Nevada showing the geographical locations of the samples. Numbered sample sites correspond to the first column in Table 1, and are a continuation of the numbers assigned to previous LLNL data by Rose et al. (1997b).

Water temperature, $\mathrm{pH}$ and conductivity values were measured in the field at the time of sample acquisition. The total dissolved inorganic carbon (TDIC, reported in $\mathrm{mg} / \mathrm{L}$ as bicarbonate) was determined in the laboratory by acidifying the water sample under vacuum and cryogenically trapping the evolved $\mathrm{CO}_{2}$ gas. Oxygen, hydrogen and carbon stable isotope ratios were measured on an isotope ratio mass spectrometer and are reported in the standard delta $(\delta)$ notation as per mil (\%o) deviations from SMOW (oxygen and hydrogen) or PDB (carbon) reference standards. Carbon-14 was measured on the accelerator mass spectrometer at LLNL, and the results are reported as percent modern carbon ( $\mathrm{pmc}$ ) relative to a NBS OX-1 standard that represents pre-nuclear testing atmosphcric $\mathrm{CO}_{2}$ abundances. More detailed descriptions of analytical methods are given in Davisson et al. (1998). 


\section{RESULTS}

\section{Oxygen and hydrogen isotopes}

Oxygen isotope results from this study were combined with data reported in Rose et al. (1997b) to create a $\delta^{18} \mathrm{O}$ contour map of south-central Nevada groundwater (Figure 3). The overall pattern is consistent with interpretations found in Davisson et al. (1998). In general, $\delta^{18} \mathrm{O}$ values are more negative at higher latitudes. Samples with $\delta^{18} \mathrm{O}$ values below $-16 \%$ are observed in the northern Toiyabe Range, and in thermal springs from the northern Big Smoky, Monitor and Antelope Valleys. Groundwater $\delta^{18} \mathrm{O}$ values between -15 and $-16 \%$ occur in the region between the Fish Creek and White Pine Ranges, and in the adjacent Railroad Valley. It is notable that alpine spring waters in the Fish Creek and White Pine Ranges have $\delta^{18} \mathrm{O}$ values that closely match those of springs emerging from the valley floor in the Railroad and Little Smoky Valleys. This suggests that the ranges are an important source of modern groundwater recharge. The relatively high precipitation rates in central Nevada are consistent with this model (cf. Lamke and Moore, 1965). On average, central Nevada receives precipitation amounts in excess of $25 \mathrm{~cm} / \mathrm{yr}$ (>10 in/yr) at elevations above $2100 \mathrm{~m}(6900 \mathrm{feet})$, and greater than $50 \mathrm{~cm} / \mathrm{yr}$ (>20 in/yr) above $2800 \mathrm{~m}$ (9200 feet).

The geographic continuity between the Railroad Valley and Pahute Mesa together with the similarity in groundwatcr stablc isotope values in both areas suggests that they may be part of the same regional flow system. The proposed flow system is represented in Figure 3 by an elongated NE-SW oriented plume of groundwater with $\delta^{18} \mathrm{O}$ values less than $-15 \%$. Assuming the Railroad Valley flow system does extend into Pahute Mesa, mixing along the flow path can readily account for the increase in $\delta^{18} \mathrm{O}$ values from around $-15.5 \%$ in the central Railroad Valley to approximately $-15.0 \%$ at Pahute Mesa. Subsurface influx from Hot Creek Valley, and local recharge from the mountains bordering the southern Railroad and Kawich Valleys are likely mixing sources. For example, groundwater from the Grant Range is considerably higher in $\delta^{18} \mathrm{O}(-14.1$ to $-14.4 \%$ ) than nearby spring discharge in the Railroad Valley. Samples collected from Hot Creek Valley during this study ranged in $\delta^{18} \mathrm{O}$ from $-13.4 \%$ to $-14.7 \%$, although deep groundwater from northern Hot Creek Valley was previously shown to have $\delta^{18} \mathrm{O}$ values as low as $-15.5 \%$ (Chapman et al., 1994).

Another pocket of low $\delta^{18} \mathrm{O}$ groundwater $(<-15 \%$ ) occurs in the Cactus Flat area, west of the Kawich Range (Figure 3). Nearby spring waters from the Kawich Range have $\delta^{18} \mathrm{O}$ values of only around $-13 \%$. If Cactus Flat is part of a larger regional flow system, the connection to recharge areas in the north is not obvious from the existing data. Davisson et al. (1998) suggested that the Cactus Flat area is a closed basin, and that the low- $\delta^{18} \mathrm{O}$ 
values represent trapped paleo-groundwater that was recharged under cooler climate conditions.

The data in Figure 3 suggest that groundwater flow beneath Pahute Mesa may continue to the southwest, contributing to groundwater discharge in Oasis Valley. This is consistent with earlier interpretations of this segment of the flow system (Blankennagel and Weir, 1973; White and Chuma, 1987; Laczniak et al., 1996) although the higher $\delta^{18} \mathrm{O}$ values in Oasis Valley require some mixing with local groundwater recharge or evaporation.

Figure 4 is a plot of $\delta^{18} \mathrm{O}$ versus $\delta \mathrm{D}$ values for samples collected during this study (triangles) together with previous data for Pahute Mesa groundwaters (circles). The significant overlap between the central Nevada and Pahute Mesa data is consistent with the proposed regional flow model. The majority of the samples have isotopic values that plot to the right of the global meteoric water line (GMWL, defined as $\delta \mathrm{D}=8 \delta^{18} \mathrm{O}+10$; see Craig, 1961). This isotopic shift is probably the result of evaporation processes (Craig et al., 1963) which are widely documented in arid environments (e.g. Gat, 1980). Although evaporation can occur as raindrops fall through unsaturated air (Friedman et al., 1962; Stewart, 1975), springtime infiltration of snowmelt is probably the most important recharge mechanism in Nevada.

Snowfall typically has $\delta^{18} \mathrm{O}-\delta \mathrm{D}$ pairs that plot on the GMWL. Figure 5 shows the isotopic variation in a single snow core collected from the Currant Summit along Hwy 6 in March 1998. Although there is considerable variability in the isotopic values of different layers, the $\delta^{18} \mathrm{O}-\delta \mathrm{D}$ pairs for each layer plot very close to the GMWL. Moreover, the integrated isotopic value of the entire core $\left(\delta^{18} \mathrm{O}=-15.5 ; \delta \mathrm{D}=-111\right)$ is consistent with the composition expected for recharge at this elevation and latitude (see model proposed by Davisson et al., 1998). Sommerfeld et al. (1991) experimentally determined that isotopic enrichment may occur during aging of the snowpack, shifting the bulk isotopic composition to the right of the GMWL. The Currant Summit snow core does not show evidence of this effect, although the recrystallized base of the snowpack is enriched in $\delta^{18} \mathrm{O}$ and $\delta \mathrm{D}$ relative to the bulk composition of the core. Friedman et al. (1991) attributed this latter effect to water vapor transport during snowpack metamorphism. Available evidence suggests that the evaporated $\delta^{18} \mathrm{O}-\delta \mathrm{D}$ signatures observed in Nevada groundwaters are inherited during and after snowpack melting due to evaporation of surface runoff and shallow groundwater (e.g. Gat and Dansgaard, 1972; Allison et al., 1984). 


\section{Carbon isotopes}

The covariation in $\delta^{13} \mathrm{C}$ versus ${ }^{14} \mathrm{C}$ values of central Nevada and Pahute Mesa groundwaters is shown in Figure 6. Pahute Mesa data are from Rose et al. (1997b). Most regional groundwaters are uniformly low in ${ }^{14} \mathrm{C}(<30 \mathrm{pmc})$, yielding uncorrected ages $>10^{4}$ years. The data show a range in $\delta^{13} \mathrm{C}$ values from approximately -11 to $-1 \%$ that is interpreted to represent chemical reactions with carbonate rock along the flow path. It is notable that the Pahute Mesa data, representing groundwater from volcanic rock aquifers, overlaps the central Nevada data, where the groundwater is predominantly from carbonate rock aquifers. Two of the Pahute Mesa groundwaters have fairly low $\delta^{13} \mathrm{C}$ values $(<-9 \%$ ) that may indicate calcite precipitation effects.

The application of correction models to obtain true ${ }^{14} \mathrm{C}$ ages is beyond the scope of this report. However, it is interesting to compare uncorrected ${ }^{14} \mathrm{C}$ ages of groundwaters from the Railroad Valley with mean groundwater age estimates from Roth and Campana (1989). The latter are based on the results of a numerical mixing-cell model for the Railroad Valley flow system. The uncorrected ${ }^{14} \mathrm{C}$ ages of Duckwater and Bull Creek Springs in the upper Railroad Valley are 10,100 and 15,500 years respectively, whereas uncorrected ${ }^{14} \mathrm{C}$ ages for Blue Eagle and Chimney Springs in the central Railroad Valley are 17,000 and 22,700 years, respectively. All of these springs are believed to originate from carbonate aquifers. Roth and Campana (1989) obtained mean age estimates for carbonate aquifer groundwaters ranging from $\sim 11,000$ to 15,000 years in the upper Railroad Valley, and 13,000 to 26,000 years in the central Railroad Valley. Although the ${ }^{14} \mathrm{C}$ ages are in relatively good agreement with the Roth and Campana model, ${ }^{14} \mathrm{C}$ correction models are expected to yield younger ages, implying mean residence times closer to the minimum age estimates of Roth and Campana.

Two samples from alpine arcas in central Nevada have relatively high ${ }^{14} \mathrm{C}$ values (85 and $92 \mathrm{pmc}$ ) and $\delta^{13} \mathrm{C}$ values near $-11 \%$ (Figure 6). These samples probably represent modern groundwater recharge (the Little Currant Creek sample was collected just downstream from a major spring). Both samples are from carbonate bedrock terrain, which accounts for the ${ }^{14} \mathrm{C}$ values $<100 \mathrm{pmc}$, and $\delta^{13} \mathrm{C}$ values that are somewhat higher than expected for $\mathrm{HCO}_{3}$ in equilibrium with biogenic soil $\mathrm{CO}_{2}$ in an area dominated by C3 vegetation (cf. Mook, 1980).

Several of the thermal springs in central Nevada are actively depositing travertine $\left(\mathrm{CaCO}_{3}\right)$. We collected travertine samples from five spring locations and measured their $\delta^{13} \mathrm{C}$ and $\delta^{18} \mathrm{O}$ values (Table 2). The $\delta^{13} \mathrm{C}$ values range from -2.3 to $+4.6 \%$, and are consistent with values expected for groundwater in equilibrium with Paleozoic carbonate rock (cf. Thomas et al., 1996, Table 17). The travertine $\delta^{18} \mathrm{O}$ values can be combined 
with measured spring water $\delta^{18} \mathrm{O}$ values to calculate precipitation temperatures using the calcite-water fractionation equation found in O'Neil et al. (1969). The calculated temperatures (Table 2) range from 8 to $30^{\circ} \mathrm{C}$ cooler than the measured temperatures at the spring orifice. This discrepancy is related to the physical process that occurs during deposition. Travertine springs typically have high $\mathrm{PCO}_{2}$ values (four of the five travertine springs sampled during this study had $\mathrm{pH}$ values $\leq 7.0$ ). As these waters reach the surface, excess $\mathrm{CO}_{2}$ tends to escape giving rise to a metastable state characterized by a progressive supersaturation with respect to $\mathrm{CaCO}_{3}$ (Turi, 1986). The $\mathrm{CO}_{2}$ exsolution process allows time for the water to cool, so that the temperature recorded by the travertine is lower than the actual groundwater temperature.

\section{DISCUSSION}

Stable isotope data presented in this study indicates that Pahute Mesa groundwaters are not derived from modern local recharge in southern Nevada. The possibility remains that the isotopic signatures represent recharge during cooler climate conditions that existed during the last ice age. However, isotopic shifts attributed to paleoclimatic conditions are equally well explained by a combination of evaporation processes and regional scale flow from recharge areas in central Nevada (see also Davisson et al., 1998). Evaporation effects are readily observable in modern groundwater throughout Nevada. Most of the small springs and creeks sampled in alpine recharge areas have $\delta^{18} \mathrm{O}-\delta \mathrm{D}$ pairs that are shifted to the right of the GMWL by an amount equivalent to the shift in regional groundwaters. Radiocarbon analyses confirm that the evaporated alpine waters are of relatively recent origin (Figure 6).

A second argument against the long-term preservation of paleoclimate isotope signatures is the relatively dynamic character of the regional flow systems. In the upper reaches of these flow systems, alpine groundwaters have stable isotope signatures that closely match those of groundwaters in the adjacent basins. This implies that modern groundwater recharge is occurring in these areas. Davisson et al. (1998) showed that subsequent mixing along regional flow paths results in systematic increases in the $\delta^{18} \mathrm{O}$ values of the groundwaters as a function of decreasing latitude. Paleoclimatic arguments are not required to explain these regional patterns. Although paleoclimatic shifts in isotopic values are likely to have occurred in Nevada, precipitation rates were probably not high enough to significantly alter the large spatial variations in groundwater $\delta^{18} \mathrm{O}$ values of the regional flow system.

If we dismiss the paleoclimate interpretation for Pahute Mesa groundwaters, then a regional flow model provides the most consistent interpretation for the data. Our results suggest that Pahute Mesa groundwater can be derived from low $\delta^{18} \mathrm{O}-\delta \mathrm{D}$ groundwater in 
the upper part of the Railroad Valley flow system by mixing with local recharge and subsurface inflow from adjacent basins. Geologic evidence suggests that regional carbonate aquifers may underlie basin fill alluvial deposits as far south as Kawich Valley (Ekren et al., 1971). To verify the continuity of this flow system, additional samples are required from the lower Railroad and Kawich Valleys. Limited data is available for wells in the lower Railroad Valley. Data from Roth and Campana (1989) show that groundwater in the southern Railroad Valley (T1N, R53E) has $\delta$ D values as low as $-118 \%$. Assuming an evaporation signature comparable to other regional groundwaters in this area, we estimate a corresponding $\delta^{18} \mathrm{O}$ value of $-15.3 \%$. This is in accord with the value expected for regional flow at this latitude. A nearby spring in the Reveille Range has a $\delta \mathrm{D}$ value of only $-97 \%$ (Roth and Campana, 1989), ruling out the likelihood of a local origin for the low $\delta \mathrm{D}$ groundwater.

Despite the apparent continuity in stable isotope values between the Railroad Valley and Pahute Mesa, the physical nature of the flow system between these areas is poorly constrained. All previous models for the Railroad Valley flow system have concluded that the system reaches its terminus at a large playa in the central Railroad Valley (Van Denburgh and Rush, 1974; Harrill et al., 1988; Prudic et al., 1995). It cannot be disputed that the playa is an important groundwater discharge area for the alluvial aquifer system (cf. Van Denburgh and Rush, 1974). Hydraulic head measurements for wells in the Railroad Valley alluvial aquifer support this conclusion (Bedinger et al., 1984; Thomas et al., 1986). However, the nature of flow through the underlying regional carbonate aquifer remains poorly understood.

The emergence of travertine-depositing thermal waters along Basin and Range faults in the central Railroad Valley suggests the carbonate aquifer may have a different hydraulic head distribution than the overlying alluvial aquifer. One of these springs (Chimney Spring) has built up an impressive travertine mound that rises more than 20 feet above the floor of Railroad Valley. The temperatures of these springs are consistent with the deep circulation of meteoric water (Olmsted et al., 1975). We can speculate that the carbonate aquifer may be confined or semi-confined in the Railroad Valley, and that groundwater is therefore transmitted southward beyond the playa, driven by hydraulic heads that are linked to recharge on carbonate rock exposures in the surrounding mountain ranges. Evidence for the nature of the confining layer is provided in a recent study by Hulen et al. (1994).

Oil fields beneath in the east-central part of the Railroad Valley playa are thought to have originated from the deep circulation of meteoric waters through the Paleozoic strata that underlies the Railroad Valley (Hulen et al., 1994). The oil-reservoir rocks are structurally located 1-2 km below the surface, at the boundary between Paleozoic carbonates and the 
overlying alluvium. Hydrothermal deposition of silica and clay minerals has formed a confining seal along this boundary, thereby trapping the hydrocarbons. The widespread precipitation of silica from thermal waters at the upper boundary of the carbonate aquifer would provide a suitable confining layer for groundwater in the carbonate aquifer. Under these circumstances, groundwater could be transported southward into the lower Railroad and Kawich Valleys. In the Kawich Valley, regional groundwater transport would presumably be transferred from the carbonate rock aquifer into the volcanic aquifers that underlie Pahute Mesa. This transition may be accompanied by the precipitation of calcite on fracture surfaces in the volcanic rocks.

Although water budget estimates for the Railroad Valley flow system suggest that groundwater recharge and discharge are largely in balance (cf. Roth and Campana, 1989), estimates of recharge rates from precipitation are in fact poorly constrained. An increase of only $1 \%$ in the estimated precipitation recharge rate would yield an additional 12,000 acre-feet of recharge each year. This is equivalent to a $10 \%$ increase in the estimated net annual water budget of 115,000 acre-feet/year (Roth and Campana, 1989). This increase would more than account for the estimated 5,500 acre-feet/year influx of groundwater into Pahute Mesa reported by Blankennagel and Weir (1973), and is well within the error margin for the hydrologic mass balance models.

\section{RECOMMENDATIONS FOR FUTURE WORK}

Environmental isotope measurements $\left(\delta^{18} \mathrm{O}, \delta \mathrm{D}, \delta^{13} \mathrm{C},{ }^{14} \mathrm{C}\right)$ were used to investigate regional groundwater flow in central and southern Nevada. The results suggest that regional flow through Pahute Mesa may originate from the Railroad Valley flow system in central Nevada. The following recommendations and proposals for future research will help to test this model.

1. Hydraulic data is needed for the Railroad Valley carbonate aquifer system, and for aquifers underlying the southern Railroad and Kawich Valleys. Environmental isotope data are needed in these same areas to verify the isotopic continuity of the proposed flow system. The development of a well that perforates the carbonate aquifer in the lower Railroad Valley would provide valuable new information to characterize the hydraulic head and aquifer transmissivity, as well as the chemical and isotopic composition of groundwater in this region.

2. The application of ${ }^{14} \mathrm{C}$ age correction models may help to constrain groundwater residence times and flow paths. This work is planned for FY99.

3. Better estimates of groundwater recharge rates from snowmelt infiltration are needed to refine hydrologic mass balance models. A study is in progress to evaluate evaporation effects in the snowpack, soil water and runoff at different times during spring recharge. Our goal is to develop a model that predicts 
recharge rates by estimating how much of the measured snowfall is lost to evaporation. The proposed model will be calibrated using existing kinetic isotope exchange models (e.g. Criss, 1987; Ingraham and Criss, 1993).

4. Additional chemical and isotopic data using different measurement techniques may provide additional insight into the nature of the regional flow system. Analysis of the major element chemistry of groundwater samples is in progress. In addition, exploratory measurements of ${ }^{36} \mathrm{Cl}$ and dissolved noble gas abundances are proposed for FY99.

5. Ongoing investigations of groundwater flow in the Oasis Valley area (funded by the UGTA program) will provide a more detailed assessment of the groundwater flow patterns at the southern end of the regional flow system.

\section{REFERENCES}

Allison, G.B., Barnes, C.J., Hughes, M.W. and Leaney, F.W.J., 1984. Effect of climate and vegetation on oxygen-18 and deuterium profiles in soils. In: Isotope Hydrology. Proceedings of the International Symposium on Isotope Hydrology and Water Resources Development, Vienna, 12-16 Sept. 1983. International Atomic Energy Agency, Vienna. p. 105-123.

Bedinger, M.S., Harrill, J.R., Langer, W.H., Thomas, J.M. and Mulvihill, D.A., 1984. Maps showing ground-water levels, springs, and depth to water, Basin and Range province, Nevada. U.S. Geol. Survey, Water Resour. Invest. Report 83-4119-B, 1:500,000, 2 sheets.

Benson, L.V. and Klieforth, H., 1989. Stable isotopes in precipitation and groundwater in the Yucca Mountain region, southern Nevada: paleoclimatic implications. In: D.H. Peterson, ed., Aspects of Climate Variability in the Pacific and Western Americas. Am. Geophys. Union, Geophysical Monograph Series, v. 55, p. 41-59.

Blankennagel, R.K. and Weir, J.E., Jr., 1973. Geohydrology of the eastern part of Pahute Mesa, Nevada Test Site, Nye County, Nevada. U.S. Geol. Survey, Prof. Paper 712-B, 35 p.

Chapman, J.B., Mihevc, T.M. and Lyles, B.F., 1994. The application of borehole logging to characterize the hydrogeology of the Faultless site, Central Nevada Test Area. Desert Research Institute, Water Resources Center, publication no. 45119, 36 p.

Claassen, H.C., 1986. Late-Wisconsin paleohydrology of the west-central Amargosa Desert, Nevada, U.S.A. Chem. Geol., v. 58, p. 311-323.

Craig, H., 1961. Isotopic variations in meteoric waters. Science, v. 133, p. 1702-1703

Craig, H., Gordon, L.I. and Horibe, Y., 1963. Isotope exchange effects in the evaporation of water. 1. Low-temperature experimental results. Jour. Geophys. Res., v. 68, p. 5079-5087.

Criss, R.E., 1987 Kinetic theory of oxygen isotopic exchange between minerals and water. Geochim. Cosmochim. Acta, vol. 51, p. 1099-1108. 
Davisson, M.L., Smith, D.K., Kenneally, J. and Rose, T.P., 1998. Isotope hydrology of southern Nevada groundwater: stable isotopes and radiocarbon. Lawrencc Livermorc National Laboratory, UCRL-JC$129475,45 \mathrm{p}$.

Dettinger, M.D., Harrill, J.R., Schmidt, D.L. and Hess, J.W., 1995. Distribution of carbonate-rock aquifers and the potential for their development, southern Nevada and adjacent parts of California, Arizona, and Utah. U.S. Geol. Survey, Water Resour. Invest. Report 91-4146, 100 p.

Eakin, T.E., 1966. A regional interbasin groundwater system in the White River area, southeastern Nevada. Water Resour. Res., v. 2, p. 251-271.

Ekren, E.B., Anderson, R.E., Rogers, C.L. and Noble, D.C., 1971. Geology of northern Nellis Air Force Base bombing and gunnery range, Nye County, Nevada. U.S. Geol. Survey, Prof. Paper 651.

Friedman, I., Machta, L. and Soller, R., 1962. Water-vapor exchange between a water droplet and its environment. Jour. Geophys. Res., v. 67, p. 2761-2766.

Friedman, I., Benson, C. and Gleason, J., 1991. Isotopic changes during snow metamorphism. In: H.P. Taylor, Jr., J.R. O'Neil and I.R. Kaplan, eds., Stable Isotope Geochemistry: A Tribute to Samuel Epstein. The Geochemical Society, Special Publication No. 3, p. 211-221.

Gat, J.R., 1980. The isotopes of hydrogen and oxygen in precipitation. In: P. Fritz and J.Ch. Fontes, eds., Handbook of Environmental Isotope Geochemistry, vol. 1, The Terrestrial Environment, A. Elsevier, Amsterdam. p. 21-47.

Gat, J.R. and Dansgaard, W., 1972. Stable isotope survey of the fresh water occurrences in Isreal and the northern Jordan Rift Valley. Jour. Hydrol., v. 16, p. 177-212.

Harrill, J.R., Gates, J.S. and Thomas, J.M., 1988. Major ground-water flow systems in the Great Basin region of Nevada, Utah, and adjacent states. U.S. Geol. Survey, Hydrologic Investigations Atlas, HA-694-C, 1:1,000,000, 2 sheets.

Hulen, J.B., Goff, F., Ross, J.R., Bortz, L.C. and Bereskin, S.R., 1994. Geology and geothermal origin of Grant Canyon and Bacon Flat oil fields, Railroad Valley, Nevada. Am. Assoc. Petroleum Geol. Bull., v. 78 , p. $596-623$.

Ingraham, N.L. and Criss, R.E., 1993. Effects of surface area and volume on the rate of isotopic exchange between water and water vapor. Jour. Geophys. Res., vol. 98, p. 20,547-20,553.

Kersting, A.B., Efurd, D.W., Finnegan, D.L., Rokop, D.J., Smith, D.K. and Thompson, J.L., 1998. Colloidal transport of plutonium in groundwater at the Nevada Test Site. Lawrence Livermore National Laboratory, UCRL-JC-128753, 14 p.

Laczniak, R.J., Cole, J.C., Sawyer, D.A. and Trudeau, D.A., 1996. Summary of hydrogeologic controls on ground-water flow at the Nevada Test Site, Nye County, Nevada. U.S. Geol. Survey, Water-Resour. Invest. Report 96-4109, 59 p.

Lamke, R.D. and Moore, D.O., 1965. Interim inventory of surface water resources of Nevada. Nevada Dept. Conserv. Nat. Resour., Water Resour. Bull. 30.

Maxey, G.B. and Eakin, T.E., 1949. Groundwater in White River valley, White Pine, Nye, and Lincoln Counties, Nevada. Nevada Dept. Conserv. Nat. Resour., Water Resour. Bull. 8. 
Mifflin, M.D. and Hess, J.W., 1979. Regional carbonate flow systems in Nevada. J. Hydrol., v. 43, p. 217-237.

Milne, W.K., Benson, L.V. and McKinley, P.W., 1987. Isotope content and temperature of precipitation in southern Nevada, August 1983-August 1986. U.S. Geol. Survey, Open-File Report 87-463, 32 p.

Mook, W.G., 1980. Carbon-14 in hydrogeological studies. In: P. Fritz and J.Ch. Fontes, eds., Handbook of Environmental Isotope Geochemistry, vol. 1. The Terrestrial Environment, A. Elsevier, Amsterdam. p. 49-74.

Olmsted, F.H., Glancy, P.A., Harrill, J.R., Rush, F.E. and Van Denburgh, A.S., 1975. Preliminary hydrogeologic appraisal of selected hydrothermal systems in nor thern and central Nevada. U.S. Geol. Survery, Open-File Report 75-56.

O'Neil, J.R., Clayton, R.N. and Mayeda, T.K., 1969. Oxygen isotope fractionation in divalent metal carbonates. Jour. Chem. Physics, v. 51, p. 5547-5558.

Prudic, D.E., Harrill, J.R. and Burbey, T.J., 1995. Conceptual evaluation of regional ground-water flow in the carbonate-rock province of the Great Basin, Nevada, Utah, and adjacent states. U.S. Geol. Survey, Prof. Paper 1409-D, 102 p.

Rose, T.P., Davisson, M.L., Hudson, G.B. and Varian, A.R., 1997a. Environmental isotope investigation of groundwater flow in the Honey Lake Basin, California and Nevada. Lawrence Livermore National Laboratory, UCRL-ID-127978, 68 p.

Rose, T.P., Kenneally, J.M., Smith, D.K., Davisson, M.L., Iudson, G.B. and Rcgo, J.H., 1997b. Chcmical and isotopic data for groundwater in southern Nevada. Lawrence Livermore National Laboratory, UCRL-ID-128000, 35 p.

Roth, J.G. and Campana, M.E., 1989. A mixing-cell modcl of the Railroad Valley regional groundwater flow system, central Nevada. Desert Research Institute, Water Resources Center, publication no. $41123,175 \mathrm{p}$.

Rush, F.E. and Everett, D.E., 1966. Water-resources appraisal of Little Fish Lake, Hot Creek, and Little Smoky Valleys, Nevada. Nevada Dept. Conserv. Nat. Resour., Water Resources - Reconnaissance Series Report 38, 38 p.

Sommerfeld, R.A., Judy, C. and Friedman, I., 1991. Isotopic changes during the formation of depth hoar in experimental snowpacks. In: H.P. Taylor, Jr., J.R. O'Neil and I.R. Kaplan, eds., Stable Isotope Geochemistry: $\Lambda$ Tribute to Samuel Epstein. The Geochemical Society, Special Publication No. 3, p. 205-209.

Stewart, J.H. and Carlson, J.E., 1978. Geologic map of Nevada. U.S. Geol. Survey, 1:500,000 scale.

Stewart, M.K., 1975. Stable isotope fractionation due to evaporation and isotopic exchange of falling waterdrops. applications to atmospheric processes and evaporation of lakes. Jour. Geophys. Res., v. 80 , p. $1133-1146$.

Thomas, J.M , Mason, J.L. and Crabtree, J.D., 1986. Ground-water levels in the Great Basin region of Nevada, Utah, and adjacent states. U.S. Geol. Survey, Hydrologic Investigations Atlas, HA-694-B, $1: 1,000,000,2$ sheets. 
Thomas, J.M., Welch, A.H. and Dettinger, M.D., 1996. Geochemistry and isotope hydrology of representative aquifers in the Great Basin region of Nevada, Utah, and adjacent states. U.S. Geol. Survey, Prof. Paper 1409-C, 100 p.

Turi, B., 1986. Stable isotope geochemistry of travertines. In: P. Fritz and J.Ch. Fontes, eds., Handbook of Environmental Isotope Geochemistry, vol. 2. The Terrestrial Environment, B. Elsevier, Amsterdam. p. 207-238.

Van Denburgh, A.S. and Rush, F.E., 1974. Water-resources appraisal of Railroad and Penoyer Valleys, east-central Nevada. Nevada Dept. Conserv. Nat. Resour., Water Resources - Reconnaissance Series Report 60,61 p.

White, A.F. and Chuma, N.J., 1987. Carbon and isotope mass balance models of Oasis Valley-Fortymile Canyon groundwater basin, southern Nevada. Water Resour. Res., v. 23, p. 571-582.

Winograd, I.J. and Thordarson, W., 1975. Hydrogeologic and hydrochemical framework, south-central Great Basin, Nevada-California, with special reference to the Nevada Test Site. U.S. Geol. Survey, Prof. Paper 712-C, $126 \mathrm{p}$. 
Table 1. Locations, field and analytical data for central Nevada water samples

\begin{tabular}{|c|c|c|c|c|c|c|c|c|c|c|c|c|c|}
\hline Sample Name & Geographic Location & Date & Section/Townshıp/Range & Latitude & Longitude & $\begin{array}{l}\text { Cond. } \\
(\mu \mathrm{S} / \mathrm{cm})\end{array}$ & $\begin{array}{c}\text { TDIC } \\
\text { (mg/L) }\end{array}$ & $\mathrm{pH}$ & $\begin{array}{l}\text { Temp } \\
\left({ }^{\circ} \mathrm{C}\right)\end{array}$ & $\begin{array}{c}\delta^{18} \mathrm{O} \\
(\% \text { SMOW) }\end{array}$ & $\begin{array}{c}\delta D \\
(\% \text { SMOW })\end{array}$ & $\begin{array}{c}\delta^{13} \mathrm{C} \\
(\% \text { PDB })\end{array}$ & $\begin{array}{c}{ }^{14} \mathrm{C} \\
\text { (pmc) }\end{array}$ \\
\hline Warm Sprnngs & Jet. Hwy 6 and 375 & 13-Мау-97 & sec. $20, T 4 N$, R50E & $38^{\circ} 11^{\prime} 16^{\prime \prime}$ & $116^{\circ} 22^{\prime} 21^{n}$ & 1322 & 1212 & 6.62 & 58.5 & -14.4 & -109 & -2.8 & \\
\hline Saulsbury Ranch Spring & Monitor Range & 13-May-97 & sec. $33, T 5 N, R 46 E$ & $38^{\circ} 14^{\prime} 16^{\prime \prime}$ & $116^{\circ} 49^{\prime} 27^{\prime \prime}$ & 1350 & & 7.80 & 20.5 & -13.2 & -106 & & \\
\hline Base Camp Well, DOD & Hot Creek Valley & 10-May-97 & sec.07, T5N, R51E & $38^{\circ} 18^{\prime} 38^{\prime \prime}$ & $116^{\circ} 16^{\prime} 41^{\prime \prime}$ & 362 & 214 & 6.52 & 18.2 & -14.0 & -109 & -7.4 & 19.7 \\
\hline Abel Spring & Railroad Valley & 13-May-97 & sec. $23, \mathrm{~T} 6 \mathrm{~N}, \mathrm{R} 54 \mathrm{E}$ & $38^{\circ} 21^{\prime} 53^{\prime \prime}$ & $115^{\circ} 52^{\prime} 01^{\prime \prime}$ & 1114 & 1048 & 6.61 & 43.5 & -15.6 & -123 & -1.9 & \\
\hline Mule Shoe Sprng & Hot Creek Range & 10-May-97 & sec.14, T6N, R49.5E & $38^{\circ} 22^{\prime} 22^{\prime \prime}$ & $116^{\circ} 25^{\prime} 46^{\prime \prime}$ & 463 & & 7.47 & 11.3 & -13.4 & -107 & & \\
\hline Rattlesnake Sprng & Pancake Range & 13-May-97 & sec.19, T7N, R52E & $38^{\circ} 26^{\prime} 59^{\prime \prime}$ & $116^{\circ} 09^{\prime} 32^{\prime \prime}$ & 300 & & 7.39 & 17.0 & .13 .4 & -107 & & \\
\hline Chımney Sprnng & Railroad Valley & 13-May-97 & sec.16, T7N, R55E & $38^{\circ} 27^{\prime} 46^{\prime \prime}$ & $115^{\circ} 47^{\prime} 35^{\prime \prime}$ & 930 & 632 & 7.00 & 65.0 & -15.7 & -122 & -1.9 & 6.4 \\
\hline Upper Warm Spring & Hot Creek Range & 10-May-97 & sec.21, T8N, R49E & $38^{\circ} 31^{\prime} 57^{\prime \prime}$ & $116^{\circ} 27^{\prime} 52^{\prime \prime}$ & 201 & 102 & 8.05 & 34.9 & -14.6 & -113 & -14.7 & \\
\hline Lockes Big Spning & Railroad Valley & 13-May-97 & sec.15, T8N. R55E & $38^{\circ} 33^{\prime} 21^{\prime \prime}$ & $115^{\circ} 46^{\prime} 15^{\prime \prime}$ & 662 & 470 & 7.67 & 36.5 & -15.4 & -124 & -2.5 & \\
\hline Blue Eagle Sprng & Railroad Valley & 12-May-97 & sec. 11, T8N, R57E & $38^{\circ} 33^{\prime} 43^{\prime \prime}$ & $115^{\circ} 31^{\prime} 41^{\prime \prime}$ & 640 & 491 & 7.04 & 27.0 & -15.1 & -116 & -5.0 & 12.9 \\
\hline The Big Well (artesıan) & Railroad Valley & $23-0 c t-97$ & sec.02, T8N, R56E & $38^{\circ} 34^{\prime} 50^{\prime \prime}$ & $115^{\circ} 38^{\prime} 15^{\prime \prime}$ & 443 & 216 & 7.80 & 22.0 & -16.6 & -125 & -6.0 & 0.7 \\
\hline Peavine Creek & Toiyabe Range & 9-May-97 & sec.30. T9N, R42E & $38^{\circ} 36^{\prime} 59^{\prime \prime}$ & $117^{\circ} 18^{\prime} 07^{\prime \prime}$ & 239 & 161 & 5.90 & 12.2 & -14.4 & -108 & -13.6 & \\
\hline N.Sixmile Canyon Sprng & Hot Creek Range & 11-May-97 & sec.35, T10N, R50E & $38^{\circ} 40^{\prime} 46^{\prime \prime}$ & $116^{\circ} 18^{\prime} 13^{\prime \prime}$ & 386 & 301 & 7.14 & 8.3 & -14.4 & -113 & -11.0 & \\
\hline Shoshone Creek & Toquima Range & 9-May-97 & sec.21, T10N, R44E & $38^{\circ} 42^{\prime} 41^{\prime \prime}$ & $117^{\circ} 02^{\prime} 45^{\prime \prime}$ & 161 & & & 8.7 & -14.9 & -112 & & \\
\hline Darroughs Hot Spring & Big Smoky Valley & 9-May-97 & sec.08, T11N, R43E & $38^{\circ} 49^{\prime} 16^{\prime \prime}$ & $117^{\circ} 10^{\prime} 48^{\prime \prime}$ & 496 & 165 & 9.08 & 92.0 & -15.5 & -121 & -10.3 & \\
\hline Little Currant Creek & White Pine Range & 13-May-97 & sec.20, T12N, R59E & $38^{\circ} 53^{\prime} 12^{\prime \prime}$ & $115^{\circ} 22^{\prime} 08^{\prime \prime}$ & 331 & 272 & 8.15 & 8.5 & -15.5 & -119 & -10.9 & 84.6 \\
\hline N.Fork Twin River & Toryabe Range & 9-May-97 & sec.16, T12N, R42E & $38^{\circ} 53^{\prime} 38^{\prime \prime}$ & $117^{\circ} 15^{\prime} 14^{\prime \prime}$ & 86 & 85 & 7.86 & 9.4 & -16.0 & -125 & -12.3 & \\
\hline E.Dobbin Summit Spring & Monitor Range & 10-May-97 & sec.31, T13N, R49E & $38^{\circ} 55^{\prime} 26^{\prime \prime}$ & $116^{\circ} 30^{\prime} 35^{\prime \prime}$ & & & & & -14.9 & -119 & & \\
\hline Sprnng, E. of Duckwater & Railroad Valley & 12-May-97 & sec.05, T12N, R56E & $38^{\circ} 55^{\prime} 41^{\prime \prime}$ & $115^{\circ} 42^{\prime} 12^{\prime \prime}$ & 665 & 384 & 7.41 & 18.5 & -15.7 & -122 & -6.3 & 29.6 \\
\hline Dianas Hot Sprng & Monitor Valley & 10-May-97 & sec.22, T14N, R47E & $39^{\circ} 01^{\prime} 47^{\prime \prime}$ & $116^{\circ} 40^{\prime} 02^{\prime \prime}$ & 608 & 362 & 6.86 & 46.9 & -16.1 & -128 & -2.8 & \\
\hline Snowball Ranch Sprıng & Antelope Range & 11-May-97 & sec.34, T14N, R51E & $39^{\circ} 02^{\prime} 23^{\prime \prime}$ & $116^{\circ} 13^{\prime} 00^{\prime \prime}$ & 279 & 210 & 6.89 & 15.3 & -15.8 & -123 & -9.8 & \\
\hline Bull Creek Spring & Railroad Valley & 12-Мay-97 & sec.25, T14N, R56E & $39^{\circ} 03^{\prime} 01^{\prime \prime}$ & $115^{\circ} 37^{\prime} 35^{\prime \prime}$ & 361 & 220 & 7.31 & 12.5 & -15.9 & -123 & -7.1 & 15.4 \\
\hline Willow Creek & Antelope Range & 11-May-97 & sec.24, T14N, R51E & $39^{\circ} 04^{\prime} 10^{\prime \prime}$ & $116^{\circ} 10^{\prime} 28^{\prime \prime}$ & 205 & & 7.61 & 15.5 & -15.3 & -123 & & \\
\hline Green Sprngs & Railroad Valley & 12-May-97 & sec.33, T15N, R57E & $39^{\circ} 06^{\prime} 57^{\prime \prime}$ & $115^{\circ} 34^{\prime} 06^{\prime \prime}$ & 432 & & 7.09 & 19.2 & -15.9 & -117 & & \\
\hline Spnng, SE of Mt. Hamilton & White Pine Range & 12-May-97 & sec.36, T16N, R57E & $39^{\circ} 12^{\prime} 41^{\prime \prime}$ & $115^{\circ} 30^{\prime} 40^{\prime \prime}$ & 473 & 368 & 7.13 & 9.1 & -15.5 & -115 & -11.4 & 91.9 \\
\hline Kingston Creek & Tolyabe Range & 9-May-97 & sec.05, T16N, R43E & $39^{\circ} 16^{\prime} 17^{\prime \prime}$ & $117^{\circ} 09^{\prime} 28^{\prime \prime}$ & 481 & 360 & 6.62 & 8.2 & -16.1 & -125 & -14.5 & \\
\hline Fish Creek Springs & Fish Creek Range & 11-May-97 & sec.08, T16N, R53E & $39^{\circ} 16^{\prime} 37^{\prime \prime}$ & $116^{\circ} 02^{\prime} 18^{\prime \prime}$ & & & & & -15.9 & -124 & & \\
\hline Spencer Hot Sprnng & Big Smoky Valley & 10-May-97 & sec. $11, T 17 N$, R45.5E & $39^{\circ} 19^{\prime} 37^{\prime \prime}$ & $116^{\circ} 51^{\prime} 17^{\prime \prime}$ & 1199 & 864 & 6.98 & 60.0 & -16.1 & -135 & -2.9 & \\
\hline Klobe Hot Spnng & Antelope Valley & 11-May-97 & sec.28, T18N, R50E & $39^{\circ} 24^{\prime} 18^{\prime \prime}$ & $116^{\circ} 20^{\prime} 50^{\prime \prime}$ & 305 & 173 & 8.97 & 65.4 & -16.6 & -130 & -12.7 & \\
\hline Faulkner Creek & Monitor Range & 11-May-97 & sec.29, T18N, R50E & $39^{\circ} 24^{\prime} 41^{\prime \prime}$ & $116^{\circ} 22^{\prime} 33^{\prime \prime}$ & 106 & & 6.26 & 13.5 & -15.9 & -123 & & \\
\hline Sulphur Spring & Pancake Range & 12-May-97 & sec.16, T18N, R56E & $39^{\circ} 25^{\prime} 24^{\prime \prime}$ & $115^{\circ} 40^{\prime} 49^{\prime \prime}$ & 552 & & 7.01 & 12.8 & -15.4 & -119 & & \\
\hline Lucky Spnngs & Diamond Range & 11-May-97 & sec.06, T18N, R54E & $39^{\circ} 27^{\prime} 30^{\prime \prime}$ & $115^{\circ} 56^{\prime} 29^{\prime \prime}$ & 341 & & 6.42 & 8.7 & -15.8 & -122 & & \\
\hline Bade Creek & Toryabe Range & 10-May-97 & sec.26, T19N, R44E & $39^{\circ} 28^{\prime} 27^{\prime \prime}$ & $117^{\circ} 00^{\prime} 05^{\prime \prime}$ & 195 & & 7.26 & 6.1 & -16.0 & -121 & & \\
\hline
\end{tabular}


Table 2. Stable isotope values and calculated precipitation temperatures of travertine deposits

\begin{tabular}{|c|c|c|c|c|c|c|}
\hline Sample Name & Date & $\begin{array}{c}\delta^{13} \mathrm{C} \text { calcite } \\
\text { (\%。 PDB) }\end{array}$ & $\begin{array}{l}\delta^{18} \text { O calcite } \\
\text { (\%o SMOW) }\end{array}$ & $\begin{array}{l}\delta^{18} \mathrm{O} \text { water } \\
(\% \circ \mathrm{SMOW})\end{array}$ & $\begin{array}{c}\text { T calculated } \\
\left({ }^{\circ} \mathrm{C}\right)\end{array}$ & $\begin{array}{c}\mathrm{T} \text { measured } \\
\left({ }^{\circ} \mathrm{C}\right)\end{array}$ \\
\hline Diana's Hot Spring, Monitor Valley & 10-May-97 & +2.1 & +11.3 & -16.1 & 30 & 47 \\
\hline Spring, E. of Duckwater, Railroad Valley & 12-May-97 & -2.3 & +12.7 & -15.7 & 25 & $33^{*}$ \\
\hline Chimney Spring, Railroad Valley & 13-May-97 & +3.5 & +8.0 & -15.7 & 50 & 65 \\
\hline Abel Spring, Railroad Valley & 13-May-97 & +4.6 & +15.2 & -15.6 & 11 & 44 \\
\hline Warm Springs, Jct. Hwy 6 and 375 & 13-May-97 & +1.7 & +9.2 & -14.4 & 51 & 59 \\
\hline
\end{tabular}

* Temperature reported for Big Warm Spring, Duckwater, the principal unmodified spring discharge zone in this area. (Van Denburgh and Rush, 1974) 


\section{FIGURE CAPTIONS}

Figure 1:

Plot of $\delta^{18} \mathrm{O}$ versus $\delta \mathrm{D}$ values for Pahute Mesa groundwater (circles) and precipitation (triangles), and spring waters from the east side of the Kawich Range. The data indicate that most Pahute Mesa groundwater cannot originate from local recharge. GMWL = global meteoric water line. Precipitation data are from Milne et al., 1987.

Figure 2:

Map of central Nevada showing locations of samples collected during this study. Numbers correlate with the first column in Table 1.

Figure 3:

Oxygen isotope contour map of groundwater samples from central and southern Nevada. Data are from this report (see also Figure 2) and from Rose et al. (1997b). Groundwater transport from the Railroad Valley to Pahute Mesa isrepresented by a NE-SW oriented plume of groundwater with $\delta^{18} \mathrm{O}$ values lessthan $-15 \%$ (shown in green).

Figure 4:

Plot of $\delta^{18} \mathrm{O}$ versus $\delta \mathrm{D}$ values of central Nevada and Pahute Mesa water samples. Significant overlap in the isotopic values of Pahute Mesa groundwater with the central Nevada data suggests a common origin. Most $\delta^{18} \mathrm{O}-\delta \mathrm{D}$ pairs plot to the right of the global meteoric water line (GMWL) due to evaporation effects.

Figure 5:

Plot of $\delta^{18} \mathrm{O}$ versus $\delta \mathrm{D}$ values for a snow core collected at the Currant Summit in eastcentral Nevada, 10 March 1998. Natural breaks in the snow pack were inferred to represent different events, and each were sampled separately. In addition, a bulk core sample was collected and analyzed. Snow density was measured volumetrically. All of the isotope data plot very close to the GMWL, although different layers exhibit dramatically different isotopic compositions. Note the isotopic enrichment toward the base of the snowpack due to recrystallization.

Figure 6:

Plot of $\delta^{13} \mathrm{C}$ versus ${ }^{14} \mathrm{C}$ values for central Nevada and Pahute Mesa water samples. The dominant sources of carbon in the system are biogenic soil $\mathrm{CO}_{2}$ (with low $\delta^{13} \mathrm{C}$ and high ${ }^{14} \mathrm{C}$ values) and carbonate rock (with high $\delta^{13} \mathrm{C}$ and low ${ }^{14} \mathrm{C}$ values). Most regional groundwaters are relatively low in ${ }^{14} \mathrm{C}(<30 \mathrm{pmc})$ with the exception of two samples collected from alpine areas. Chemical reactions with carbonate minerals along the flow path causes a concomitant increase in $\delta^{13} \mathrm{C}$ with decreasing ${ }^{14} \mathrm{C}$ values. It is notable that the central Nevada (Railroad Valley flow system) and Pahute Mesa data show significant overlap in carbon isotope compositions despite differences in aquifer lithologies, suggesting hydrologic continuity. 


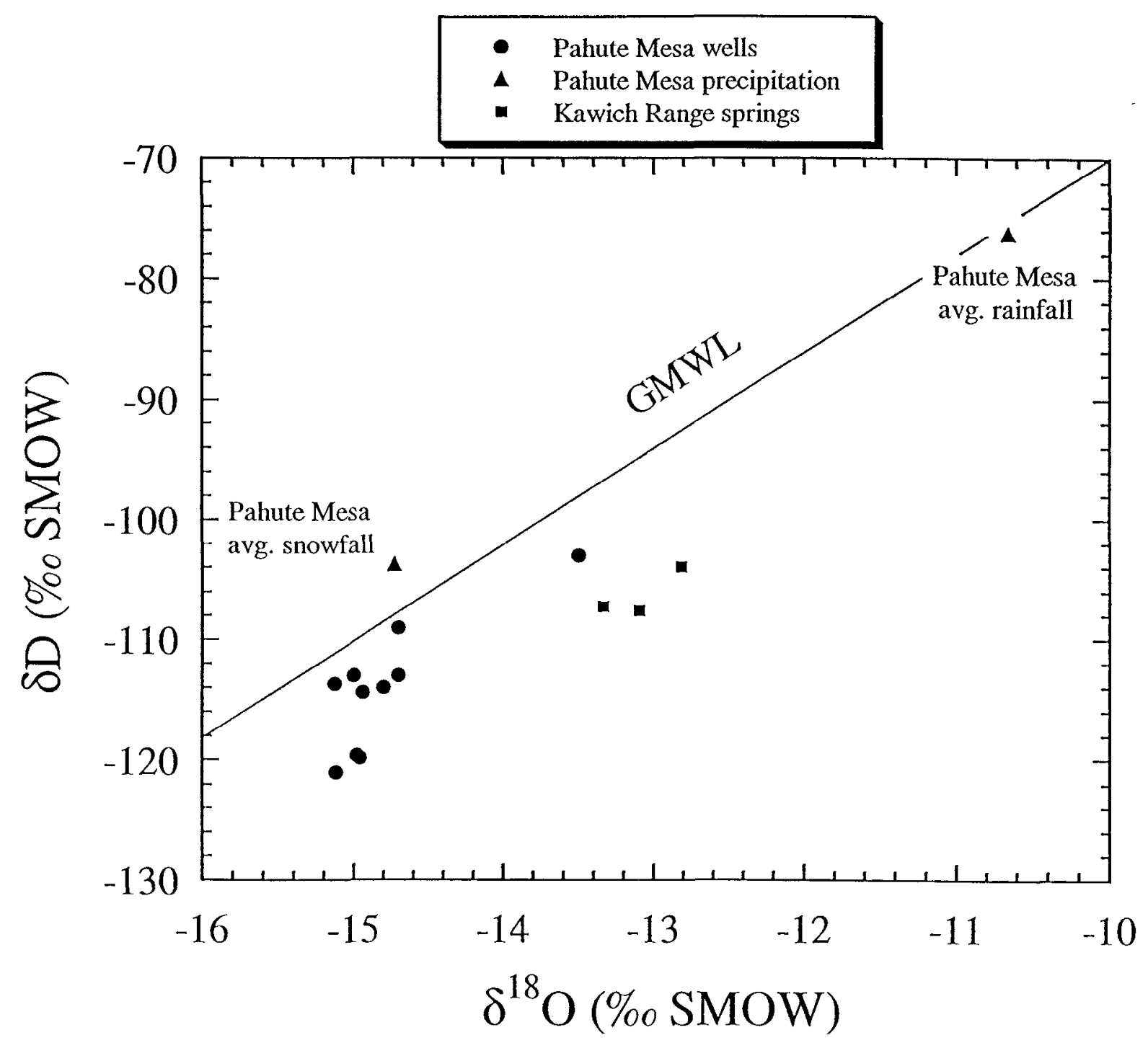

Figure 1 


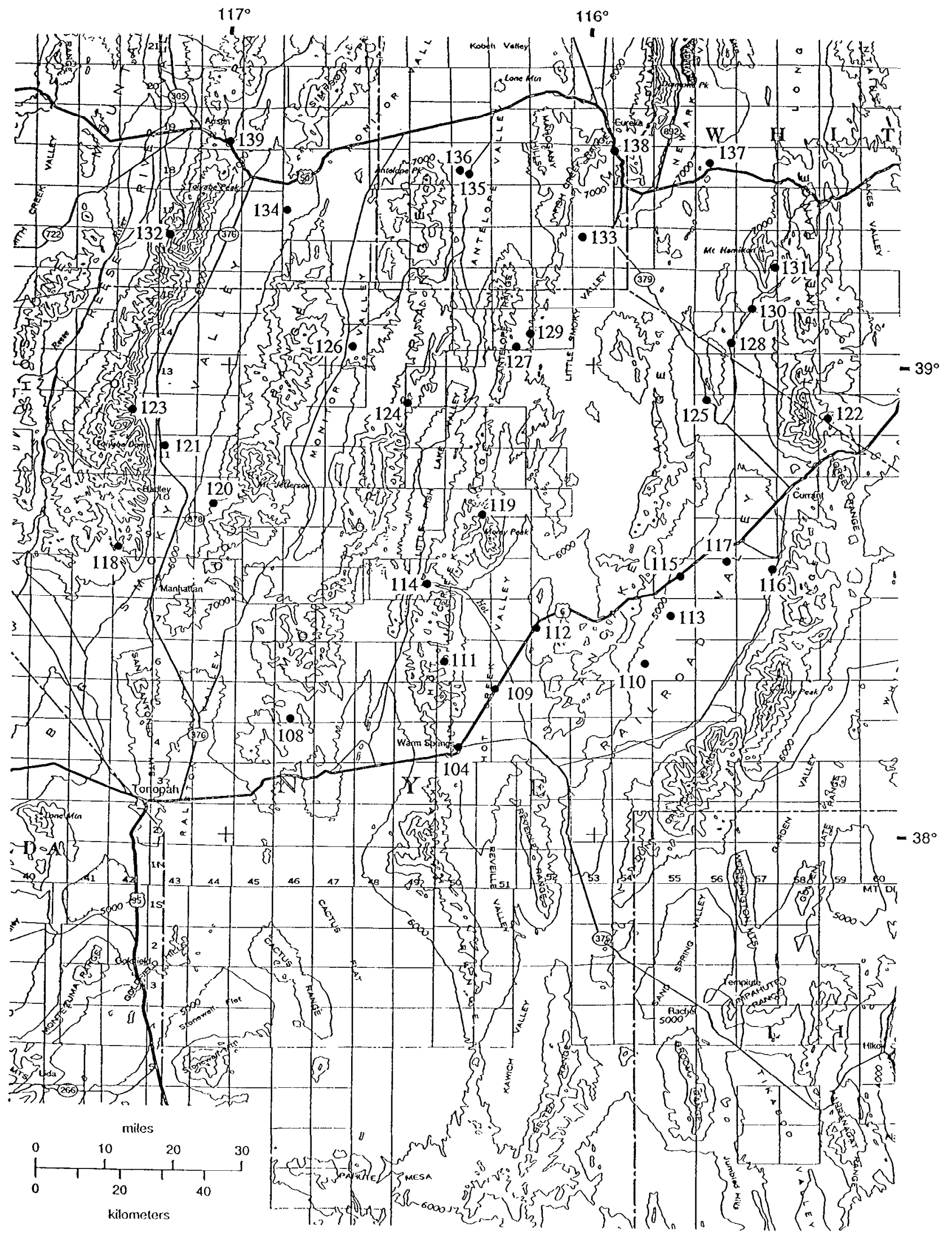

Figure 2 


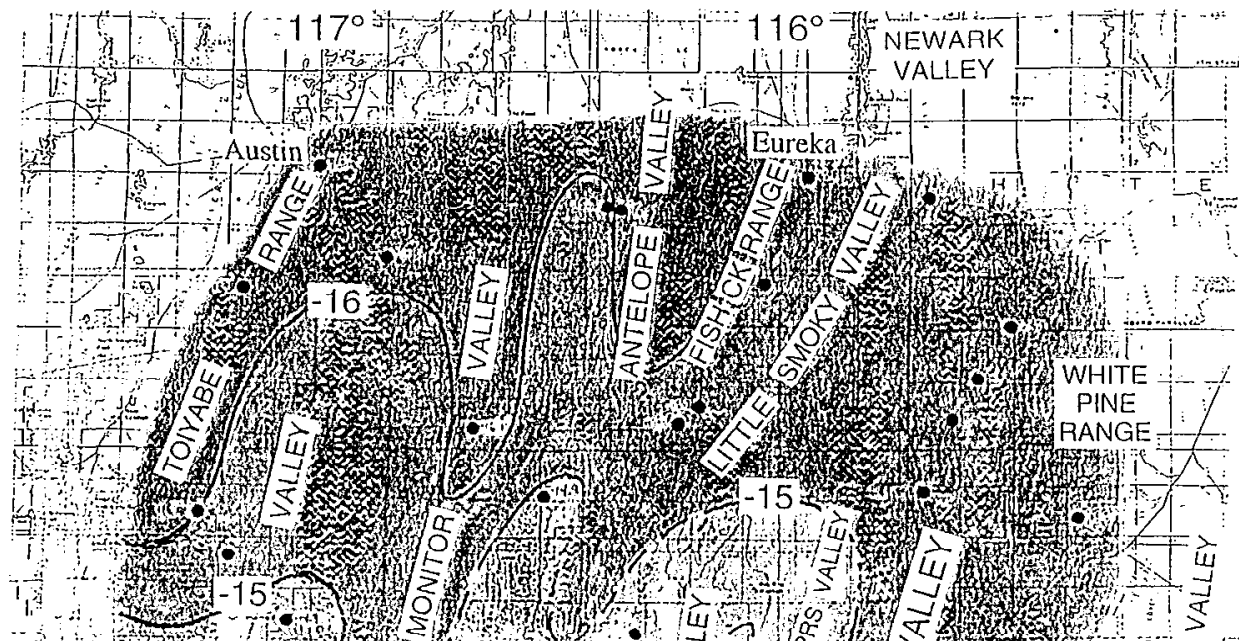

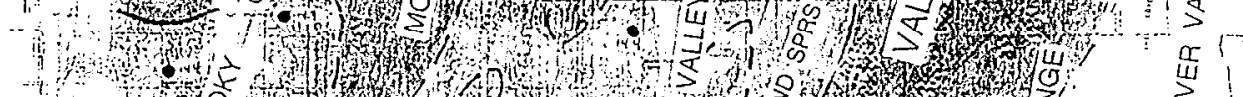

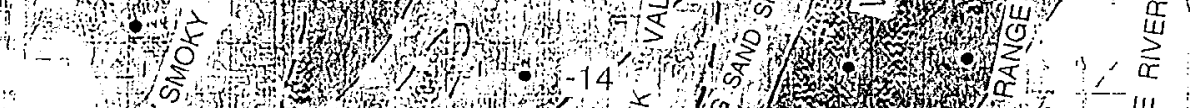

4
$i$ $\Rightarrow \quad$ o

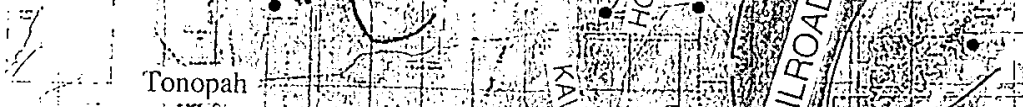

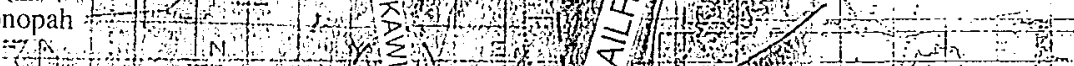

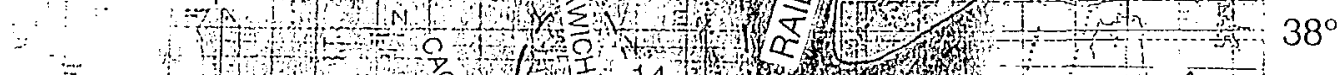

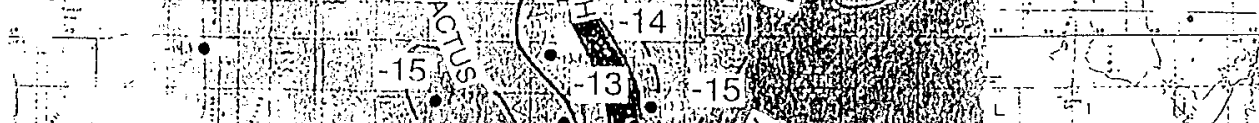

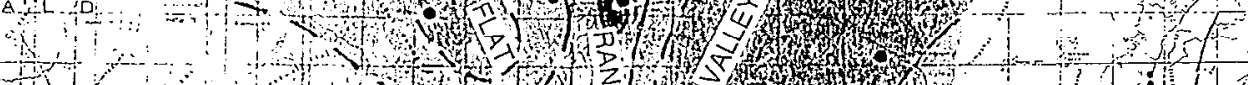
T1

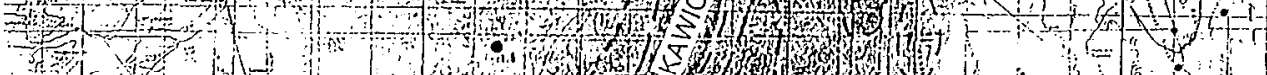
为

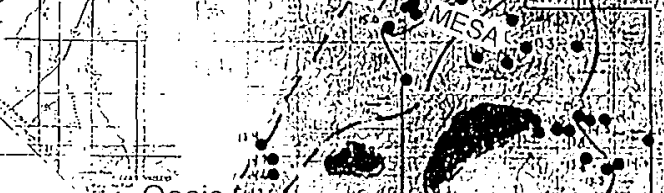

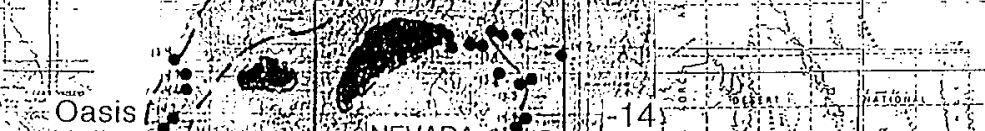
$04-14 \mathrm{r}^{-0}$ -

$\delta^{18} \mathrm{O}(\% \circ \mathrm{SMOW})$

miles

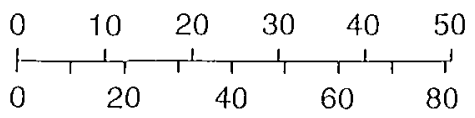

kilometers 


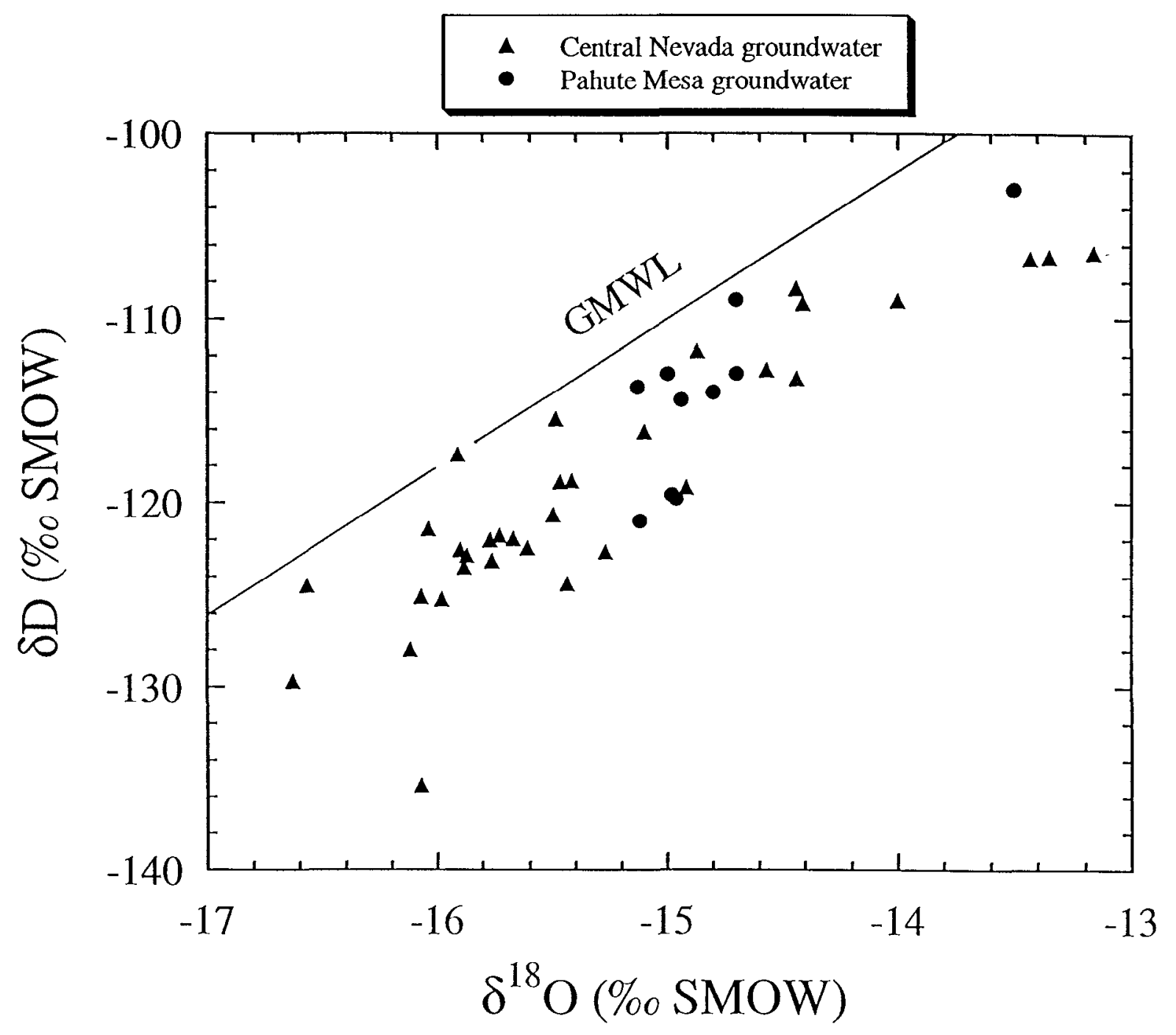

Figure 4 


\section{Currant Summit Snow Core}

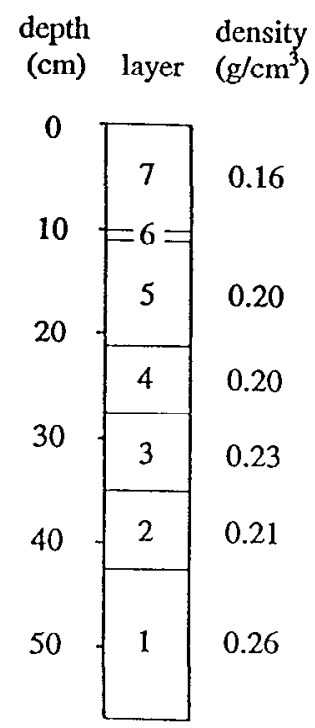

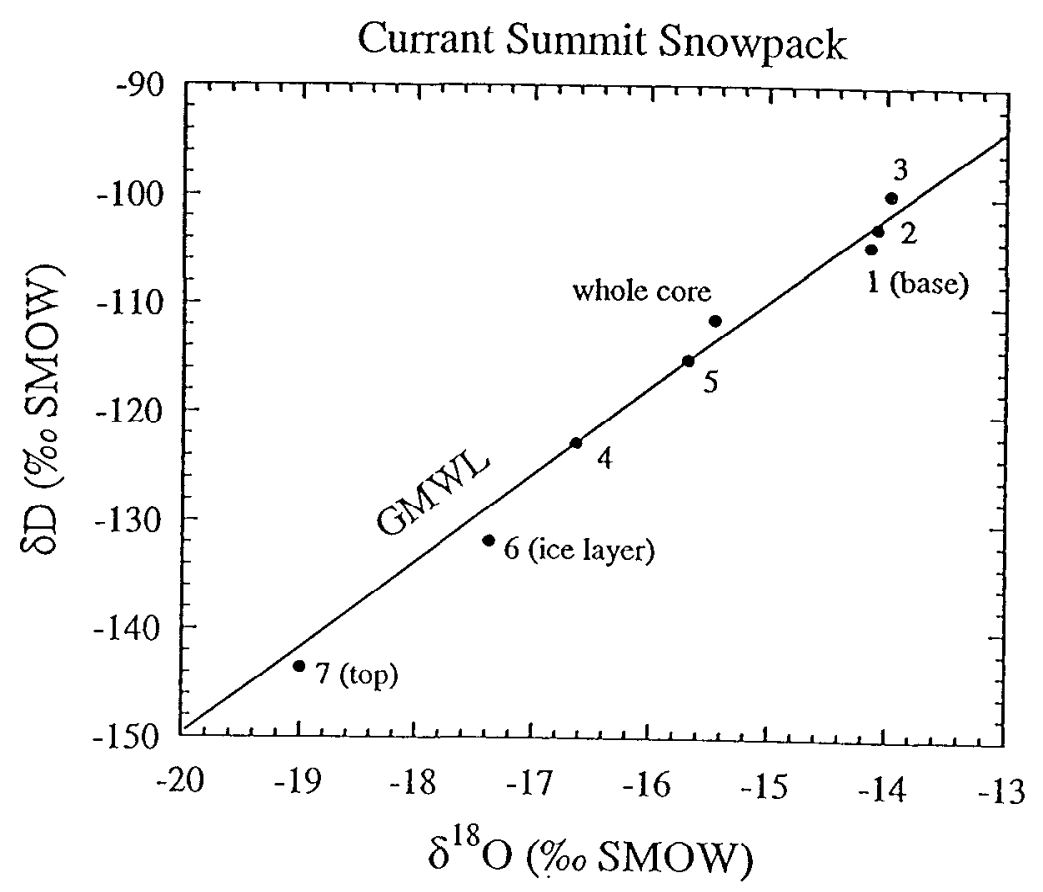

Figure 5 


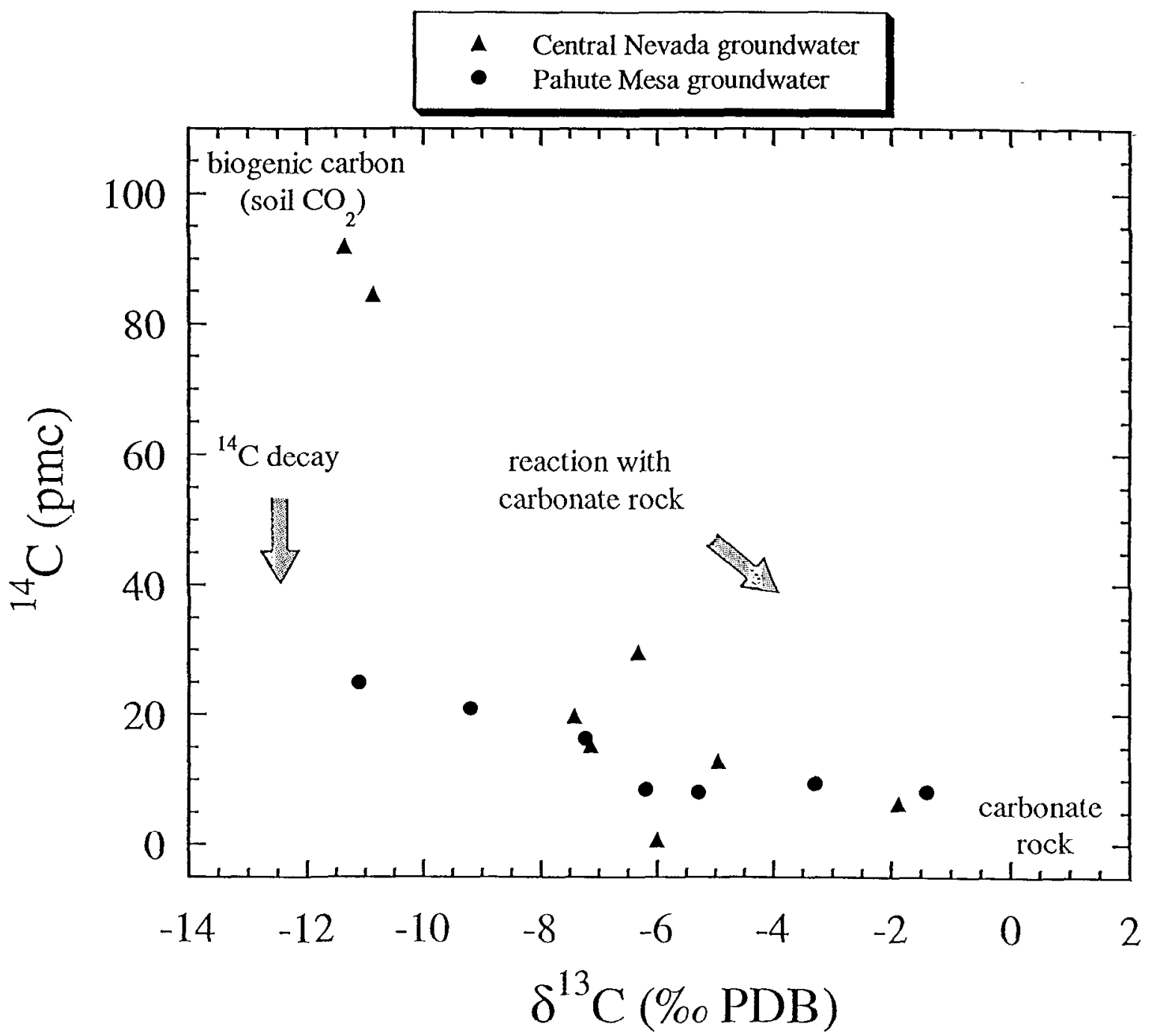

Figure 6 


\title{
CHAPTER 7
}

\section{MIGRATION OF PLUTONIUM IN GROUNDWATER AT THE NEVADA TEST SITE}

\author{
Joint Collaboration by LANL* and LLNL
}

Annie B. Kersting, D. Wes Efurd*, David L. Finnegan*, Donald J. Rokop*, David K. Smith, and Joseph L. Thompson*

\begin{abstract}
Radionuclide migration in groundwater was evaluated by sampling groundwater pumped from a well cluster penetrating two aquifers downgradient from an underground nuclear event at the Nevada Test Site. Tritium, and low concentrations of cobalt, cesium, europium and plutonium isotopes were detected. Groundwater samples were filtered and various particle fractions characterized and analyzed for radionuclides. Except for tritium, greater than $95 \%$ of the measured radioactivity was associated with particulate and colloidal fractions consisting of predominately clays and zeolites. The ${ }^{240} \mathrm{Pu} / 239 \mathrm{Pu}$ isotopic ratio of groundwater from both aquifers is the same and uniquely matches the BENHAM nuclear event, located $1.3 \mathrm{~km}$ to the north. Plutonium was transported a significant distance through fractured volcanic rock.
\end{abstract}

\section{INTRODUCTION}

Significant quantities of $\mathrm{Pu}$ have been introduced into the environment worldwide as a result of use in nuclear weapons testing, nuclear weapons production, and nuclear power plant operations. Therefore, understanding the fate and transport of radionuclides in groundwater is of great scientific and public concern. In order to predict major pathways and mechanisms for transport, as well as design containment and cleanup methods, it is essential to understand under what conditions radionuclides may migrate in the geosphere. Radionuclide transport in groundwater is a function of a variety of factors including, radionuclide speciation, groundwater composition, host rock composition and aquifer hydrologic characteristics.

In this study, we characterize the nature and extent of radionuclide migration in groundwater collected from well cluster, ER-20-5, located approximately $300 \mathrm{~m}$ west of the underground TYBO nuclear test cavity and $1300 \mathrm{~m}$ south of the BENHAM nuclear test cavity on Pahute Mesa at the NTS (Figure 1A). We specifically address the role of colloids in radionuclide transport hosted in fractured volcanic rock aquifers. Colloids are small, less than 1 micron particles that naturally occur suspended in groundwater and are usually comprised of either inorganic (mineral fragments and secondary minerals of the host rock), or organic material. Oxides and hydroxides of actinide elements can also form as colloids. Due to their small size, colloids have the potential to be transported with groundwater. We use the isotopic ratios of plutonium to uniquely identify the 
source of plutonium and demonstrate that the vast majority of the measured radioactivity is associated with the colloidal clays and zeolites present in the groundwater. In this report we summarize our results. Some information about the Pu concentrations in groundwater at this site has already been published (Thompson et al, 1997) (Kersting et. al., in press) This report summarizes the most current data and elaborates on some of the experimental details.

\section{GEOLOGICAL AND FIELD DESCRIPTION OF THE STUDY AREA}

The study area is located in the northwestern portion of the NTS where thick sequences of ashflow tuffs and rhyolitic lava flows dominate the geology (Blankennagel and Weir, 1973) (Laczniak et al., 1996). The volcanic rocks are fractured and faulted and form important hydrologic units. Most of the faults strike north-northeast and north, reflective of Basin and Range faulting. Hydrologic gradients in Pahute Mesa suggest a southwestward and southward flow of groundwater. Estimated groundwater flow velocities are not well constrained and range from 1 to $80 \mathrm{~m} /$ year (Blankennagel and Weir, 1973).

ER-20-5 well cluster was drilled approximately $280 \mathrm{~m}$ downgradient of the T'YBO nuclear event (Figure 1A). Well ER-20-5\#1 was completed and screened in a transmissive aquifer at a depth of 701-784 m, comparable to the detonation location (working point) of the TYBO event. Well ER20-5 \#3 was completed and screened in different transmissive aquifer at 1046-1183 $\mathrm{m}$ at a depth comparable to the working point of the BENHAM event, located $1300 \mathrm{~m}$ to the north. Well ER20-5 \#2 was abandoned and never completed. Figure 1B shows a cross-section of the BENHAM and TYBO underground nuclear events relative to the ER-20-5 well cluster. The TYBO nuclear event was fired on Pahute Mesa 14 May 1975 at a depth of $765 \mathrm{~m}$ (2509 ft), well below the static water table at $630 \mathrm{~m}(2066 \mathrm{ft})$. TYBO had a thermonuclear yield between 200 and $1000 \mathrm{kt}$. The BENHAM event was fired on Pahute Mesa 19 December 1968 at a depth of $1402 \mathrm{~m}(4599, \mathrm{ft})$ well below the static water table at $641 \mathrm{~m}(2102 \mathrm{ft})$. BENHAM had an announced yield of $1.15 \mathrm{Mt}$. Other nuclear tests in the area were BELMONT and MOLBO located east of TYBO.

\section{ANALYTICAL PROCEDURES}

Groundwater from each aquifer was pumped into 200-L drums on three separatc occasions over a 16 month period, and sent to the laboratory for analysis. Unfiltered groundwater samples were analyzed for tritium $\left({ }^{3} \mathrm{H}\right)$, gamma-emitting radionuclides and plutonium isotopes. Additional 200$\mathrm{L}$ groundwater samples from well \#1, collected on the second and third sampling efforts, were subjected to a series of filtration experiments. The groundwater was serial filtered using $1000 \mathrm{~nm}$, $50 \mathrm{~nm}$ and 100,000 nominal molecular weight $(\sim 7 \mathrm{~nm})$ filter sizes. The particulate material $(>$ $1000 \mathrm{~nm}$ ), the colloidal fractions (between 1000-50 nm, 50-7 nm) and the ultrafiltrate or dissolved fraction $(<\sim 7 \mathrm{~nm})$ were collected and analyzed for ${ }^{3} \mathrm{H}$, gamma-emitting radionuclides and plutonium isotopes. 
Tritium was measured by liquid scintillation counting. The groundwater samples were evaporated to dryness and the residue counted with an intrinsic germanium detector system to identify gammaemitting radionuclides. The particulate and colloidal fractions were ashed and gamma counted prior to plutonium analysis. The plutonium was analyzed by both alpha spectrometry and thermal ionization mass spectrometry. The TIMS technique is a much more sensitive technique and can measure accurately the ${ }^{240} \mathrm{Pu} / 239 \mathrm{Pu}$ ratio and the concentration of $\mathrm{Pu}$ to better than $10^{-12} \mathrm{~g}$ in our 200-L samples. Upon completion of the gamma ray analyses the residues were spiked with ${ }^{242} \mathrm{Pu}$ and dissolved in a mixture of $\mathrm{HF}, \mathrm{HNO}_{3}$, and $\mathrm{HCLO}_{4}$ acids. The samples were evaporated to dryness and re-dissolved in $7 \mathrm{~N} \mathrm{HNO}_{3}$. Plutonium isolation and purification was accomplished by anion exchange chromatography. The chemically purified plutonium fraction from each sample was electroplated on platinum and measured by alpha spectroscopy. Next, the plutonium was removed from the platinum disks and the ${ }^{240} \mathrm{Pu} / 239 \mathrm{Pu}$ atom ratios were measured by thermal ionization mass spectrometry (Perrin, 1985 ).

A concerted effort was made to eliminate the possibility that either field or laboratory contamination had occurred. To test for laboratory contamination, we analyzed blanks both with the reagents used in our TIMS analyses, and with Pu-free water. Laboratory procedural blanks had plutonium concentrations $(<2 \mathrm{pg})$ significantly below the concentrations analyzed. The levels of Pu detected were orders of magnitude too small to affect our analyses and had isotopic ratios different from our samples. Surface soil samples were collected in the vicinity of the BENHAM and TYBO nuclear events and their ${ }^{240} \mathrm{Pu} / 239 \mathrm{Pu}$ isotopic ratios were measured.

Archived melt glass material collected from the cavity region immediately after the detonation of the BENHAM and TYBO events was re-analyzed for its ${ }^{240} \mathrm{Pu} / 239 \mathrm{Pu}$ isotopic composition and compared to data previously obtained during the U. S. nuclear test program. Splits from the melt glass were analyzed at LANL as well as Lawrence Livermore National Laboratory (LLNL) to provide external laboratory comparison. The procedure for measuring Pu from the solid samples is the same as mentioned above.

\section{RESULTS}

The results of tritium and gamma analyses are given in Table I and Table II. The concentrations of $\mathrm{Pu}$ (by weight and by activity) are given in Table III. We have reported the combined values for ${ }^{239} \mathrm{Pu}$ and ${ }^{240} \mathrm{Pu}$. The contribution of ${ }^{238} \mathrm{Pu}$ is about $5 \%$ additional activity and an insignificant weight addition. The half-lives of ${ }^{239} \mathrm{Pu}$ and ${ }^{240} \mathrm{Pu}$ are very long, so there is no need to correct our data to a to reference time.

Figures $2 \mathrm{~A}$ and $\mathrm{B}$ show the radioactivity detected in the unfiltered groundwater from well \#1 and well \#3, respectively for the April, 1997 sampling. The levels of radioactivity measured from 
groundwater collected in well \#3 are significantly lower (1000 to 20 times lower) than those measured in groundwater from well \#1. Eu isotopes were not detected in groundwater from well \#3 but may be present below the level of detection $\left(\sim 1 \mathrm{E}^{-3} \mathrm{~Bq} / \mathrm{L}\right)$. A comparison of the levels of radioactivity detected in the groundwater collected from each sampling period for well \#1 is shown on Figure 2C. Data were decay corrected to April, 1997. The same suite of radionuclides were detected in each groundwater sample and the levels of radioactivity were similar. Although not shown, the same is true of groundwater from well \#3.

Except for ${ }^{3} \mathrm{H}$, the levels of radioactivity for ${ }^{60} \mathrm{Co},{ }^{137} \mathrm{Cs}$ and europium isotopes measured in the groundwaters are below the drinking water limits for a given radionuclide calculated using Environmental Protection Agency's (EPA) regulatory requirements for community drinking water (Environmental Protection Agency, 1991). The maximum permissible limit in the drinking water standard is based on a whole body dose or organ dose limit of $4 \mathrm{mrem} /$ year calculated using the assumption of drinking 2 liters of contaminated water a day for a year (EPA regulations 40 CFR 141.16 ).

The ${ }^{3} \mathrm{H}$ concentration was essentially unaltered in passing through the filters. In contrast, greater than 99 percent of the europium and plutonium isotopes measured in the groundwater from well \#1 were associated with the colloidal and particulate fractions (Figure 3A). About ninety-one percent of the cobalt (Co) and ninety-five percent of the cesium (Cs) was in the particulate/colloid fractions.

The ${ }^{240} \mathrm{Pu} / 239 \mathrm{Pu}$ isotopic ratios after a nuclear detonation are unique to a specific event, and as a result, we can use the plutonium isotopic composition to fingerprint the source of the plutonium measured in the groundwater detected in both aquifers. The actual Pu isotope ratios measured on groundwaters sampled from ER-20-5 are not stated in this report. Pu ratios measured on post-shot event material are classified and event specific. Therefore, ratios were omitted in order to keep this report unclassified. Figure $3 \mathrm{~B}$ is a comparison of the ${ }^{240} \mathrm{Pu} / 239 \mathrm{Pu}$ isotopic ratio measured in the groundwater from well \#1 normalized to other ${ }^{240} \mathrm{Pu} / 239 \mathrm{Pu}$ isotopic ratios measurcd in this study. The ${ }^{240} \mathrm{Pu} / 239 \mathrm{Pu}$ isotopic ratio of the unfiltered groundwater in well \#1 matches that measured in groundwater from well \#3, that measured from the colloidal fraction from well \#1 and the BENHAM event. The $240 \mathrm{Pu} / 239 \mathrm{Pu}$ isotopic ratio of the groundwater does not match the TYBO, MOLBO and BELMONT nuclear events.

Plutonium was present at very low concentrations ( $<4 \mathrm{Pu} / \mathrm{gm}$ of sample) in the soil samples. The ${ }^{240} \mathrm{Pu} / 239 \mathrm{Pu}$ isotopic ratio of the soil samples has a distinctly different ratio than the groundwater. This eliminated the possibility that the Pu measured in the water samples collected at ER-20-5 was the result of surface contamination during sampling. 
Finally, we analyzed archived debris provided by LLNL from both TYBO and BENHAM. Both LANL and LLNL did the post shot debris analyses independently using different methodologies. Results of the plutonium isotopic analyses of the archived melt glass from BENHAM and TYBO from each laboratory agree to within 1.5 percent and also match the original values measured immediately following the nuclear tests. Obtaining the same results from two different laboratories eliminated the possibility of laboratory bias.

By determining the source of the plutonium, we have shown for the first time that plutonium has been transported in groundwater an appreciable distance from the nuclear test site where it originated. The ${ }^{240} \mathrm{Pu} /{ }^{239} \mathrm{Pu}$ isotopic results show that plutonium measured in the groundwater from well \#1 and well \#3 is from the same source and that source is BENHAM, $1.3 \mathrm{~km}$ north of the ER-20-5 well cluster.

As with the cobalt, cesium and europium radionuclides, the plutonium was associated with the particulates and colloids in the groundwater. The different size fractions were analyzed by $\mathrm{X}$-ray diffraction (XRD) and a scanning electron microcscope (SEM). The colloidal material was composed of clays (illite and smectite), zeolites (mordenite and clinoptilolite/heulandite), and cristobalite. Clinoptilolite and heulandite are closely related zeolites but are not easily distinguished on the basis of optical properties, XRD patterns or composition. No attempt has been made to distinguish between the two minerals. The same mineral assemblage was detected in all three size fractions ( $>1000 \mathrm{~nm},>50 \mathrm{~nm}$ and $\sim 7 \mathrm{~nm}$ ). Figure 4 shows SEM images of the two distinct morphologies observed; the zeolite and clay. As a result of the small size of the colloids, quantitative data could not be obtained by SEM or electron microprobe. Due to the distinctive morphology of illite and mordenite, we suggest the flat platy minerals are illite and the rod shaped minerals are mordenite.

\section{DISCUSSION}

The presence of ${ }^{3} \mathrm{H},{ }^{137} \mathrm{Cs}$ and ${ }^{60} \mathrm{Co}$ in groundwater adjacent to a nuclear test cavity was expected (Smith et al., 1996); the presence of the Eu and Pu isotopes in water from well \#1 was not.

Europium isotopes are relatively insoluble and have not often been detected in groundwater at the NTS; when they have been found they have been associated with colloids (Buddemeier and Hunt, 1988).

The data obtained are consistent with plutonium and other radionuclides having been transported in groundwater as colloidal material. Although plutonium has been shown experimentally to strongly sorb to clays and zeolites and form a "pseudocolloid", plutonium can also exist as an "intrinsic colloid" composed of a plutonium oxide (Silva and Nitsche, 1995). Both types of colloids have the capacity to be transported by groundwater. From this study, we cannot distinguish the colloidal form of the plutonium and further study is needed. Cobalt, cesium and europium have 
not been observed experimentally to form intrinsic colloids and we suggest that these radionuclides sorb onto the colloidal sized clays and zeolites as pseudocolloids and are transported in the groundwater.

Clays and zeolites are common secondary minerals in altered rhyolitic tuff and smectite, clinoptilolite and mordenite have specifically been identified in the rocks on Pahute Mesa (Blankennagel and Weir, 1973) and elsewhere in volcanic tuffs on the NTS (Broxton et al., 1987). The colloidal minerals identified in ER-20-5 groundwater from well \#1 are consistent with the secondary minerals observed in the host aquifer. Previous characterization studies of naturally occurring colloids in groundwater collected from volcanic tuff aquifers located in the southwestern part of the NTS are composed of minerals similar to those found in this study; clays and zeolites (Viani and Martin, 1996)(Kingston and Whitbeck, 1991).

Clays and zeolites have a high sorption capacity for actinides and other cationic radionuclides. In some cases, the capacity of radionuclides to sorb to specific minerals is well known. For example, strontium, cesium, cobalt and actinides (including plutonium) have been shown to strongly sorb to clays and zeolites as a result of their large cation-exchange capacity (Comans and Hockley, 1992) (Torstenfelt et al., 1988) (Triay et al., 1996). The experimentally determined distribution coefficient, $\mathrm{K}_{\mathrm{d}}$, for sorption of plutonium $(\mathrm{Pu}(\mathrm{V})$ ) onto clinoptilolite (75 to $500 \mathrm{~mm}$ size range) using NTS water ( $\mathrm{pH} 7$ ) and a plutonium concentration of $\sim 1 \mathrm{E}-9 \mathrm{M}$ is greater than 500 . These results indicate that plutonium sorbs significantly onto clinoptilolite. Preliminary results of sorption/desorption experiments of plutonium onto the clay, montmorillonite, suggest that this process is irreversible on the time scale of the laboratory experiments (Triay et al., 1997).

The maximum concentration of plutonium at the ER-20-5 site is about $1 \mathrm{E}-14 \mathrm{M}$, which is lower than solubility limits experimentally determined ( E-8 M) for plutonium (V) species likely to be present in NTS groundwater (Nitsche et al., 1993) and near the solubility limits calculated ( $1 \mathrm{E}-12 \mathrm{M}$ to $1 \mathrm{E}-17 \mathrm{M}$ ) assuming thermodynamic equilibrium for plutonium (IV) (Stout and Leider, 1994) (Guillaumont and Adloff, 1992). Due to the very low concentration of plutonium measured in the groundwater at ER-20-5, it is unlikely that the plutonium was above the solubility limit and precipitated as a solid phase. Our results showing greater than 99 percent of the plutonium was detected in the filterable fraction of the groundwatcr in conjunction with previous sorption experimental work are consistent with plutonium having been transported in groundwater as colloidal material and not as a dissolved phase.

We do not know how far downgradient of the ER-20-5 site radionuclides have moved, therefore cannot determine the exact extent of Pu migration. Nor do we know the exact distribution of plutonium immediately after the detonation of BENHAM in 1968. Based on 40 years of re-drilling underground test cavities and collecting melt glass samples for nuclear test diagnostics, it is thought that the overwhelming majority of the refractory radionuclides such as plutonium are 
incorporated into the melt glass at the bottom of the test cavity. In some events, small amounts of melt glass were found in the chimney, presumably melt that was splashed or extruded along fractures as the chimney collapsed shortly after detonation (Levy, 1972). It is possible that a small fraction of the plutonium may have been injected through fractures beyond the melt glass region (vertically or horizontally) immediately after the detonation. Radionuclide transport as a result of fracture injection elsewhere on the NTS has been observed up to a maximum of a few hundred meters from the working point. Yet, the possibility of fracture injection over a distance of greater than a kilometer seems highly unlikely. However, some fraction of the observed plutonium may have been initially injected through fractures up to several hundred meters and subsequently transported by groundwater. Given these uncertainties, we conservatively assume the plutonium has migrated a minimum of $1 \mathrm{~km}$ in 28 years to estimate a migration rate of approximately $35 \mathrm{~m} / \mathrm{y}$.

The ER-20-5 well cluster was thought to lie downgradient from the TYBO nuclear event (U. S. Department of Energy, 1997) but clearly the plutonium isotopic ratios measured in the groundwater from ER-20-5 do not match the plutonium isotopic ratio from the TYBO event (Figure 3B). In addition, there is no evidence that the plutonium detected in the groundwater is a mixture from the TYBO and BENHAM nuclear events. Even mixing a small amount of groundwater with the plutonium isotopic ratio from TYBO with the plutonium isotopic composition of BENHAM would result in an isotopic ratio between the TYBO and BENHAM values. This is not observed. We suspect the ER-20-5 well cluster is not downgradient of the TYBO event.

The radioactivity measured in the groundwater from the shallower aquifer (well \#1) is significantly grcater than mcasured in the decper aquifer; yet the shallow aquifer lies $\sim 600 \mathrm{~m}$ stratigraphically above the working point of the BENHAM event where the melt glass resides. The fracture flow hydrodynamics in this area are unknown. A previous study at the Cheshire nuclear event concluded that as a result of the blast, the groundwater that was previously flowing at the cavity depth had been re-routed, moving upward to an overlying more permeable zones via the rubble chimney (Buddemeier and Hunt, 1988). It is not known if this occurred at BENHAM, but may explain why the radionuclides were detected in both aquifers sampled at the ER-20-5 well cluster.

We regard the observation of $\mathrm{Pu}$ in groundwater at this location as extremely significant. To our knowledge this is the first time $\mathrm{Pu}$ has been shown to be transported by groundwater and for a significant distance. The other radionuclides detected $\left({ }^{3} \mathrm{H},{ }^{60} \mathrm{Co},{ }^{137} \mathrm{Cs}, 152,154,155 \mathrm{Eu}\right)$ cannot be shown to originate from any particular shot, although we presume that europium (often used as a chemical analog for actinides) probably came to the BENHAM site by the same path as the Pu. We have demonstrated that the $\mathrm{Pu}$ and other radionuclides are associated with the colloidal fraction of the groundwater.

Is the concentration of $\mathrm{Pu}$ found at ER-20-5 dangerous from the perspective of human risk? The maximum concentration we measured for $239,240 \mathrm{Pu}$ was $6.3 \mathrm{E}-1 \mathrm{pCi} / \mathrm{L}$. The maximum permissible 
concentrations limits in drinking water based on regulations in 40 CFR 141.16 and in DOE Order 5400.5. The maximum permissible values he calculated based on these regulations were $1.55 \mathrm{E} 0$ $\mathrm{pCi} / \mathrm{L}$ and 5.81E1 $\mathrm{pCi} / \mathrm{L}$, respectively (IT, 1996). Our measured Pu concentrations are below these calculated drinking water limits.

The ability to measure accurately the plutonium isotopic ratios of groundwater collected from aquifers downgradient of radionuclides deposited in the saturated subsurface and match these ratios to a specific nuclear event makes this study truly unique. For the first time we have determined that plutonium has been transported in groundwater in fractured volcanic rock a significant distance. The radionuclides were associated with the particulate and colloidal fractions and not the dissolved fraction. We believe that the radionuclides either sorb to the clay and zeolite minerals, or in the case of plutonium, may migrate as a plutonium oxide. This work demonstrates that plutonium is not immobile in the subsurface as previously believed. We caution that modeling radionuclide transport based solely on solubility arguments will severely underestimate the transport of radionuclides associated with colloids.

\section{SUMMARY}

- Tritium, ${ }^{60} \mathrm{Co},{ }^{137} \mathrm{Cs}, 152,154,155 \mathrm{Eu}$ and $\mathrm{Pu}$ isotopes were detected in groundwaters pumped from wells ER20-5 \#1 and \#3.

- The concentrations of radionuclides are much lower in groundwater from well ER20-5 \#3 than measured in groundwater from well ER20-5 \#1.

- The Pu isotopic ratios in groundwater from wells ER20-5 \#1 and \#3 is the same and matches the BENHAM nuclear event located $1.3 \mathrm{~km}$ to the north.

- Pu was transported a significant distance through fractured volcanic rock.

- Greater than $95 \%$ of the radioactivity measured is associated with the colloidal fraction of the groundwater.

- The colloidal fraction is composed of clays, zeolites and minor cristobalite minerals; minerals that have high sorption capacity for radionuclides.

- There is no evidence that the Pu detected is from TYBO. We suggest that groundwater direction in the vicinity of BENHAM-TYBO area is more southward that previously believed. 


\section{REFERENCES}

Blankennagel, R.K. and Weir, J.E.J., 1973, Geohydrology of the Eastern Part of Pahute Mesa, Nevada Test Site, Nye County, Nevada.

Broxton, D.E., Bish, D.L. and Warren, D.L., 1987, Distribution and Chemistry of Diagenetic Minerals at Yucca Mountain, Nye County, Nevada, Clays and Clay Minerals 35, 89-110.

Buddemeier, R. W. and Hunt, J. R., 1988, Transport of Colloidal Contaminants in Groundwater: Radionuclide Migration at the Nevada Test Site, Applied Geochemistry 3:535-548.

Comans, R.N.J. and Hockley, D.E., 1992, Kinetics of Cesium Sorption on Illite, Geochim. Cosmochim. Acta 56, 1157-1164.

Environmental Protection Agency, 1991, National Primary Drinking Water Regulations, Proposed Rules, 40 CFR 141.16.

Guillaumont, R. and Adloff, J.P., 1992, Behavior of Environmental Plutonium at very Low Concentrations, Radiochim. Acta 58/59, 53-60.

IT Corporation, Ed. Adams, S., 1996, UGTA Subcommittee on Source Terms: Dose Evaluation for Individuals Ingesting Radiologically Contaminated Groundwater" internal report to Janet Wille, IT Corporation, Las Vegas, NV.

Kersting, A.B., Efurd, D.W., Finnegan, D.L., Rokop, D.J., Smith, D.K. and Thompson, J.L., 1998, Plutonium Migration in Groundwater at the Nevada Test Site, Submitted Nature

Kingston, W.L. and Whitbeck, M., 1991, Characterization of Colloids Found in Various Groundwater Environments in Central and Southern Nevada.

Laczniak, R.J., Cole, J.C., Sawyer, D.A., and Trudeau, D.A., 1996, Summary of Hydrogeologic Controls on Ground-Water Flow at the Nevada Test Sitc, Nye County, NV. U.S. Geological Survey.

Levy, H.B., 1972, On Evaluating the Hazards of Groundwater Contamination by Radioactivity from an Underground Nuclear Explosion. Lawrence Livermore National Laboratory report UCRLID-51278.

Perrin, R.E., 1985, International Journal of Mass Spectrometry and Ion Physics 64, 17.

Silva, R.J. and Nitsche, H., 1995, Actinide Environmental Chemistry, Radiochim. Acta 70/71, 377-396.

Nimz, G. J. and Thompson, J. L., 1992, Underground Radionuclide Migration at the Nevada Test Site, US Department of Energy/Nevada Field Office report DOE/NV-346.

Nitsche, H., Gatti, R.C., Standifer, E.M., Lee, S.C., Muller, A., Prussin, T., Deinhammer, R.S., Maurer, H., Becraft, K., Leung, S. and Carpenter, S.A., 1993, Measured Solubilities and Speciations of Neptunium, Plutonium, and Americium in a Typical Groundwater (J-13) from the Yucca Mountain Region Milestone Report 3010-WBS 1.2.3.4.1.3.1.

Smith, D. K., Nagle, R.J, Kenneally,J. M., 1996, Transport of Gaseous Fission Products Adjacent to an Underground Nuclear Test Cavity, Radiochimica Acta. 73:177-183. 
Smith, D. K. Ed., 1997, Hydrologic Resources Management Program FY 1996 Progress Report, Lawrence Livermore National Laboratory UCRL-ID-126886.

Smith, D. K., Esser, B. K., Kenneally, J. M., Nagle, R. J., Rego, J. H., Royval, J. L., 1996, Hydrologic Resources Management Program FY 1995 Progress Report, Lawrence Livermore National Laboratory, UCRL-ID-123486.

Stout, R.B. and Leider, H., 1994, Preliminary Waste Form Characteristics Report Version 1.0.

Thompson, J. L., 1996, Radionuclide Distribution in a Nuclear Test Cavity: the Baseball Event, Radiochimica Acta 72:157-162.

Thompson, J. L. Ed., 1997, Laboratory and Field Studies Related to the Hydrologic Resources Management Program October 1, 1995 -- September 30, 1996, Los Alamos National Laboratory report LA-13270-PR.

Torstenfelt, B., Rundberg, R.S. and Mitchell, A.J., 1988, Actinide Sorption on Granites and Minerals as a Function of $\mathrm{pH}$ and Colloids/Pseudocolloids, Radiochim. Acta 44/45, 111-117.

Triay, I.R., Cotter, C.R., Kraus, S.M., Huddleston, M.H., Chipera, S.J. and Bish, D.L., 1996, Radionuclide Sorption in Yucca Mountain Tuffs with J-13 Well Water: Neptunium, Uranium, and Plutonium. Los Alamos National Laboratory report LA-12956-MS.

Triay, I.R., Lu, N., Cotter, C.R. and Kitten, H.D., 1997, Iron Oxide Colloid Facilitated Plutonium Transport in Groundwater, in: American Chemical Society, pp.

U. S. Department of Encrgy, 1997, Completion Report of Well Cluster ER-20-5.

Viani, B.E. and Martin, S.I., 1996, Groundwater Colloid Characterization, Lawrence Livermore National Laboratory. 
TABLE I

Gamma Emitters in ER-20-5 \#1 Water

\begin{tabular}{|c|c|c|c|c|c|}
\hline \multirow[t]{2}{*}{ Radionuclid } & \multicolumn{2}{|c|}{ Activity at count time } & \multicolumn{2}{|c|}{$\begin{array}{c}\text { Activity at } t_{0}= \\
15 \text { May } 75\end{array}$} & \multirow[t]{2}{*}{$\%$ Error } \\
\hline & $\mathrm{Bq} / \mathrm{L}$ & $\mathrm{pCi} / \mathrm{L}$ & $\mathrm{Bq} / \mathrm{L}$ & $\mathrm{pCi} / \mathrm{L}$ & \\
\hline $\begin{array}{c}3 \mathrm{Jan} 96 \text { afte } \\
\text { pumping of } 5.0 \\
\text { gals }\end{array}$ & & & & & \\
\hline $3_{\mathrm{H}}$ & $2.44 \mathrm{E} 6$ & $6.6 \mathrm{E} 7$ & $7.8 \mathrm{E} 6$ & $2.11 \mathrm{E} 8$ & \pm 2 \\
\hline${ }^{137} \mathrm{Cs}$ & $4.4 \mathrm{E}-1$ & $1.19 \mathrm{E} 1$ & $7.1 \mathrm{E}-1$ & $1.93 \mathrm{E} 1$ & \pm 2 \\
\hline${ }^{60} \mathrm{Co}$ & $6.9 \mathrm{E}-2$ & $1.9 \mathrm{E} 0$ & $1.08 \mathrm{E} 0$ & $2.9 \mathrm{E} 1$ & \pm 4 \\
\hline${ }^{152} \mathrm{Eu}$ & $5.8 \mathrm{E}-2$ & $1.6 \mathrm{E} 0$ & $1.68 \mathrm{E}-1$ & $4.5 \mathrm{E} 0$ & \pm 4 \\
\hline${ }^{154} \mathrm{Eu}$ & $7.0 \mathrm{E}-2$ & $1.88 \mathrm{E} 0$ & $3.8 \mathrm{E}-1$ & $1.02 \mathrm{E} 1$ & \pm 5 \\
\hline${ }^{155} \mathrm{Eu}$ & $1.7 \mathrm{E}-2$ & $4.7 \mathrm{E}-1$ & $3.6 \mathrm{E}-1$ & $9.8 \mathrm{E} 0$ & \pm 10 \\
\hline $\begin{array}{c}3 \mathrm{Jun} 96 \text { aft } \\
\text { pumping of } 4.0 \\
\text { gals }\end{array}$ & & & & & \\
\hline${ }^{3} \mathbf{H}$ & $2.52 \mathrm{E} 6$ & $6.81 \mathrm{E} 7$ & $8.24 \mathrm{E} 6$ & $2.23 \mathrm{E} 8$ & \pm 2 \\
\hline${ }^{137} \mathrm{Cs}$ & $5.7 \mathrm{E}-1$ & $1.55 \mathrm{E} 1$ & $9.34 \mathrm{E}-1$ & $2.52 \mathrm{E} 1$ & \pm 2 \\
\hline${ }^{60} \mathrm{Co}$ & $6.8 \mathrm{E}-2$ & $1.8 \mathrm{E} 0$ & $1.11 \mathrm{E} 0$ & $3.0 \mathrm{E} 1$ & \pm 4 \\
\hline${ }^{152} \mathrm{Eu}$ & $5.6 \mathrm{E}-2$ & $1.5 \mathrm{E} 0$ & $1.67 \mathrm{E}-1$ & $4.5 \mathrm{E} 0$ & \pm 4 \\
\hline${ }^{154} \mathrm{Eu}$ & $6.3 \mathrm{E}-2$ & $1.70 \mathrm{E} 0$ & $3.6 \mathrm{E}-1$ & $9.3 \mathrm{E} 0$ & \pm 5 \\
\hline${ }^{155} \mathrm{Eu}$ & $1.6 \mathrm{E}-2$ & $4.4 \mathrm{E}-1$ & $3.6 \mathrm{E}-1$ & $9.3 \mathrm{E0}$ & \pm 10 \\
\hline $\begin{array}{r}22 \text { Apr } 97 \text { af } \\
\text { pumping of } 5 \\
\text { gals } \\
\end{array}$ & & & & & \\
\hline${ }^{3} \mathrm{H}$ & $2.55 \mathrm{E} 6$ & $6.89 \mathrm{E} 7$ & $8.80 \mathrm{E} 6$ & $2.37 \mathrm{E} 8$ & \pm 4 \\
\hline${ }^{137} \mathrm{Cs}$ & $5.8 \mathrm{E}-1$ & $1.57 \mathrm{E} 1$ & $9.6 \mathrm{E}-1$ & $2.60 \mathrm{E} 1$ & \pm 1 \\
\hline${ }^{60} \mathrm{Co}$ & $6.2 \mathrm{E}-2$ & $1.7 \mathrm{E} 0$ & $1.13 \mathrm{E} 0$ & $3.1 \mathrm{E} 1$ & \pm 3 \\
\hline $152 \mathrm{Eu}$ & $5.1 \mathrm{~F}-2$ & $1.4 \mathrm{E} 0$ & $1.6 \mathrm{E}-1$ & $4.3 \mathrm{E} 0$ & \pm 4 \\
\hline${ }^{154} \mathrm{Eu}$ & $5.8 \mathrm{E}-2$ & $1.6 \mathrm{E} 0$ & $3.5 \mathrm{E}-1$ & $9.4 \mathrm{E} 0$ & \pm 7 \\
\hline $155_{\mathrm{Eu}}$ & $1.1 \mathrm{E}-2$ & $3.1 \mathrm{E}-1$ & $2.9 \mathrm{E}-1$ & $7.9 \mathrm{E} 0$ & \pm 15 \\
\hline
\end{tabular}


TABLE II

Gamma Emitters in ER-20-5 \#3 Water

\begin{tabular}{|c|c|c|c|c|c|}
\hline \multirow[t]{2}{*}{ Radionuclide } & \multicolumn{2}{|c|}{ Activity at count time } & \multicolumn{2}{|c|}{$\begin{array}{c}\text { Activity at } t_{0}= \\
14 \text { May } 75\end{array}$} & \multirow[t]{2}{*}{ \% Error } \\
\hline & $\mathrm{Bq} / \mathrm{L}$ & $\mathrm{pCi} / \mathrm{L}$ & $\mathrm{Bg} / \mathrm{L}$ & $\mathrm{pCi} / \mathrm{L}$ & \\
\hline $\begin{array}{c}15 \text { Feb } 96 \text { after } \\
\text { pumping of } 8.00 E 3 \\
\text { gals }\end{array}$ & & & & & \\
\hline${ }^{3} \mathrm{H}$ & $2.4 \mathrm{E} 3$ & $6.5 \mathrm{E} 4$ & $7.8 \mathrm{E} 3$ & $2.1 \mathrm{E} 5$ & \pm 4 \\
\hline${ }^{137} \mathrm{Cs}$ & $6.4 \mathrm{E}-3$ & $1.7 \mathrm{E}-1$ & $1.04 \mathrm{E}-2$ & $2.8 \mathrm{E}-1$ & \pm 7 \\
\hline $60_{\mathrm{Co}}$ & $2 \mathrm{E}-3$ & $5 \mathrm{E}-2$ & $3 \mathrm{E}-2$ & $8 \mathrm{E}-1$ & \pm 20 \\
\hline $\begin{array}{c}31 \mathrm{Jul} 96 \text { after } \\
\text { pumping of } 5.31 \mathrm{E} 6 \\
\text { gals } \\
\end{array}$ & & & & & \\
\hline${ }^{3} \mathrm{H}$ & $5.6 \mathrm{E} 3$ & $1.46 \mathrm{E} 5$ & $1.9 \mathrm{E} 4$ & $5.0 \mathrm{E} 5$ & \pm 4 \\
\hline${ }^{137} \mathrm{Cs}$ & $5.3 \mathrm{E}-3$ & $1.4 \mathrm{E}-1$ & $8.7 \mathrm{E}-3$ & $2.3 \mathrm{E}-1$ & \pm 15 \\
\hline${ }^{60} \mathrm{Co}$ & $3 \mathrm{E}-3$ & $9 \mathrm{E}-2$ & $6 \mathrm{E}-2$ & $1.5 \mathrm{E} 0$ & \pm 20 \\
\hline $\begin{array}{c}22 \text { Apr } 97 \text { after } \\
\text { pumping of } 3.8 E 4 \\
\text { gals } \\
\end{array}$ & & & & & \\
\hline $3 \mathrm{H}$ & $5.3 \mathrm{E} 3$ & $1.42 \mathrm{E} 5$ & $1.81 \mathrm{E} 4$ & $4.9 \mathrm{E} 5$ & \pm 4 \\
\hline${ }^{137} \mathrm{Cs}$ & $2 \mathrm{E}-3$ & $6 \mathrm{E}-2$ & $4 \mathrm{E}-3$ & $1 \mathrm{E}-1$ & \pm 20 \\
\hline${ }^{60} \mathrm{Co}$ & $2 \mathrm{E}-3$ & $6 \mathrm{E}-2$ & $4 \mathrm{E}-2$ & $1 \mathrm{E} 0$ & \pm 30 \\
\hline
\end{tabular}

TABLE III

Plutonium $(239+240)$ Concentrations in Water at ER-20-5

\begin{tabular}{|l|l|l|l|l|l|l|}
\hline Date Sampled & \multicolumn{3}{|c|}{$\# 1$ Well } & \multicolumn{3}{c|}{ \#3 Well } \\
\hline & $\mathrm{g} / \mathrm{L}$ & $\mathrm{pCi} / \mathrm{L}$ & $\mathrm{Bq} / \mathrm{L}$ & $\mathrm{g} / \mathrm{L}$ & $\mathrm{pCi} / \mathrm{L}$ & $\mathrm{Bq} / \mathrm{L}$ \\
\cline { 2 - 7 } & $6.59 \mathrm{E}-12$ & $5.3 \mathrm{E}-1$ & $2.0 \mathrm{E}-2$ & & & \\
3 Jan 1996 Feb 1996 & & & & & \\
3 Jun 1996 & $3.24 \mathrm{E}-12$ & $2.6 \mathrm{E}-1$ & $9.7 \mathrm{E}-3$ & & & \\
31 July 1996 & & & & $1.01 \mathrm{E}-13$ & $8.0 \mathrm{E}-3$ & $3.0 \mathrm{E}-4$ \\
22 April 1997 & $7.82 \mathrm{E}-12$ & $6.3 \mathrm{E}-1$ & $2.3 \mathrm{E}-2$ & $1.41 \mathrm{E}-13$ & $8.5 \mathrm{E}-3$ & $3.2 \mathrm{E}-4$ \\
\hline
\end{tabular}




\section{FIGURE CAPTIONS}

Figure 1:

(A) Map of the Nevada Test Site, NV USA with the location of all underground nuclear tests detonated between 1957 and 1992. Enlarged map of the field area in Pahute Mesa showing the location of the well cluster ER-20-5 (Wells \#1 and \#3) and the nearby underground nuclear events. (B) A-A' is a north-south cross-section projecting the BENHAM and TYBO nuclear events relative to the ER-20-5 well cluster. Well \#3 is located approximately $30 \mathrm{~m}$ south of well \#1. Both BENHAM and TYBO events created chimneys that propagated to the ground surface. The working point (WP) denotes the depth of the nuclear device before detonation. The nuclear melt glass coalesces below the WP at the bottom of the cavity. The radius of the cavity is a function of the nuclear yield, density of the rock type and depth of burial (distance from ground surface to WP).

Figure 2:

Comparison of radioactivity detected in unfiltered groundwater samples from ER-20-5 well cluster, wells \#1 and \#3. For the purposes of comparison, all data were decay corrected to 22 April, 1997, the time of the third sampling. (A) Concentration of radioactivity measured in unfiltered groundwater from the shallower aquifer, pumped from well \#1. The only gamma-emitting radionuclides detected were, ${ }^{60} \mathrm{Co},{ }^{137} \mathrm{Cs},{ }^{152} \mathrm{Eu},{ }^{154} \mathrm{Eu},{ }^{155} \mathrm{Eu}$. (B) Concentration of radioactivity measured in unfiltered groundwater from the deeper aquifer, pumped from well \#3. Note that the concentrations measured in water from the deeper aquifer are much lower than those measured from water in well \#1. (C) Comparison of the radioactivity detected in the three groundwater samples collected from well $\# 1$ over the duration of the field experiment. The first sample was collected after $1.89 \mathrm{E} 1 \mathrm{~m}^{3}$ (5,000 gallons) were pumped; the second after $1.52 \mathrm{E} 3 \mathrm{~m}^{3}$ (401,000 gallons). The pumps were shut down, and restarted the next spring. An additional $2.23 \mathrm{E} 2 \mathrm{~m}^{3}$ (59,000 gallons) of groundwater was pumped before the third sample was collected. A total of $1.76 \mathrm{E} 3 \mathrm{~m}^{3}$ were pumped from well \#1 and $2.19 \mathrm{E} 3 \mathrm{~m}^{3}$ from well \#3.

Figure 3:

(A) Comparison of the radioactivity collected on the different filter sizes and the ultrafiltrate fraction. Data are normalized to the total radioactivity measured in the unfiltered water. The ultrafiltrate is the water that passed through all the filters $(<\sim 7 \mathrm{~nm})$. (B) Comparison of the ${ }^{240} \mathrm{Pu} /{ }^{239} \mathrm{Pu}$ isotopic ratios measured in the groundwater from well \#1 divided by the measured ${ }^{240} \mathrm{Pu} /{ }^{239} \mathrm{Pu}$ isotopic ratios of the different samples listed on the $\mathrm{X}$ axis. Values used are averages: ER-20-5 \#1, Number of samples averaged, $(N)=3$; ER-20-5 \#3, N=2; Colloids, N=4; TYBO, $\mathrm{N}=6$; BENHAM, $\mathrm{N}=12 ;$ MOLBO, $\mathrm{N}=7$; BELMONT, $\mathrm{N}=4$; and Soil, $\mathrm{N}=2$.

Figure 4:

SEM photographs showing (A) the tabular, lath shaped morphology of the zeolite, mordenite, and (B) the platy appearance of the clay, illite. The two distinct morphologies were observed in all three size fractions ( $>1 \mu \mathrm{m}, 1000 \mathrm{~nm}-50 \mathrm{~nm}$ and $50 \mathrm{~nm}-\sim 7 \mathrm{~nm}$ ).

\section{ACKNOWLEDGMENTS}

We appreciate the contributions of B.A. Martinez, F.R. Roensch J.W. Chamberlin, and G.P. Russ III for sample analysis. Thought provoking discussions with I.R. Triay, B.E. Viani, R.W. Lougheed, J.F. Wild, and G.B. Hudson are greatly appreciated. 


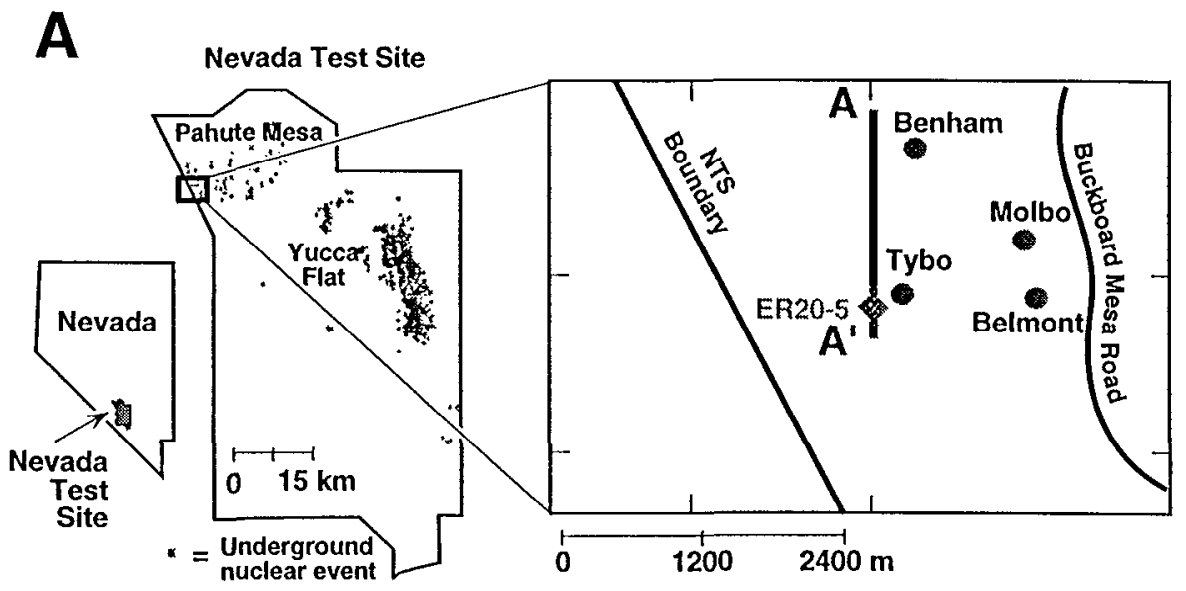

B

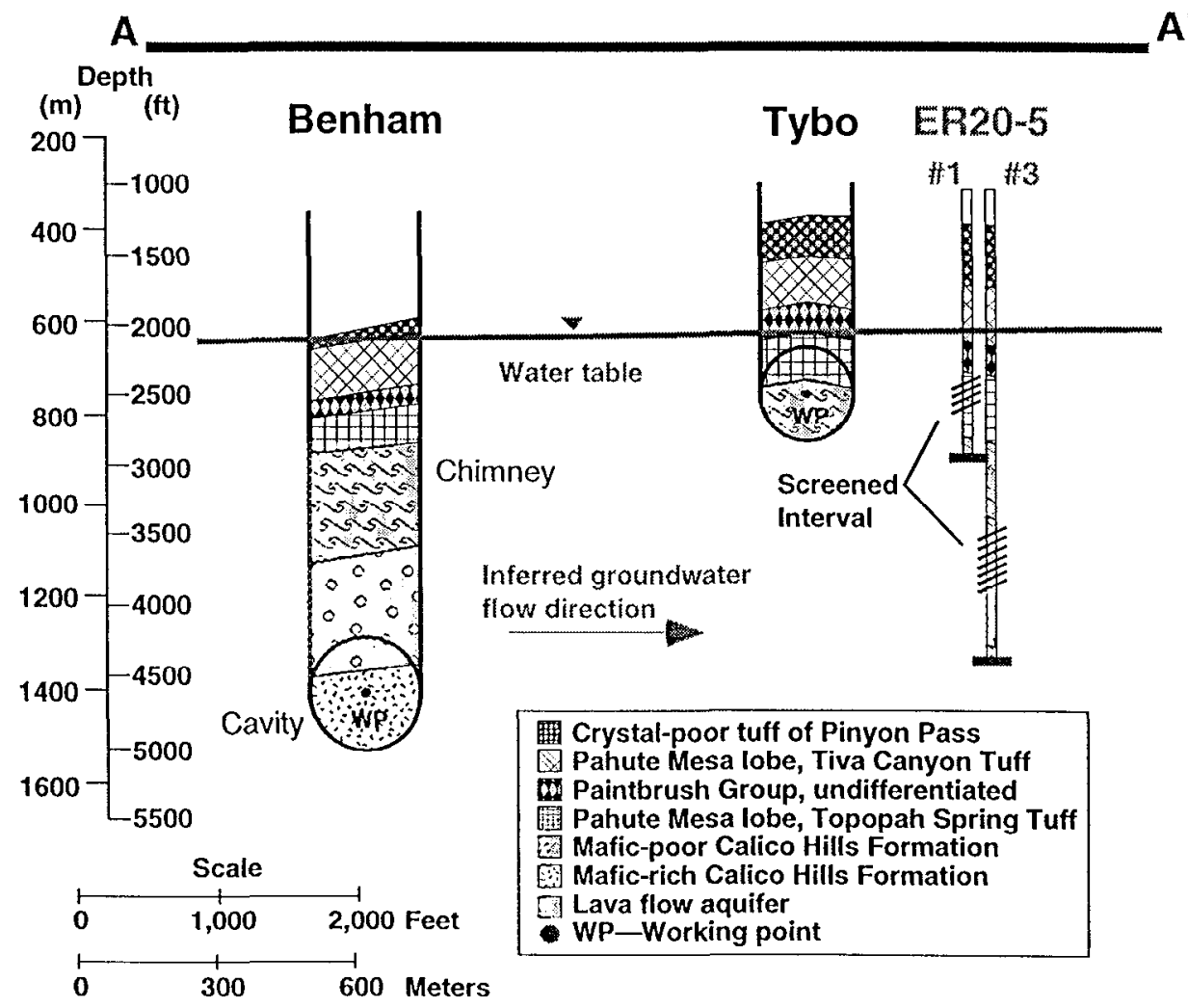

Figure 1 

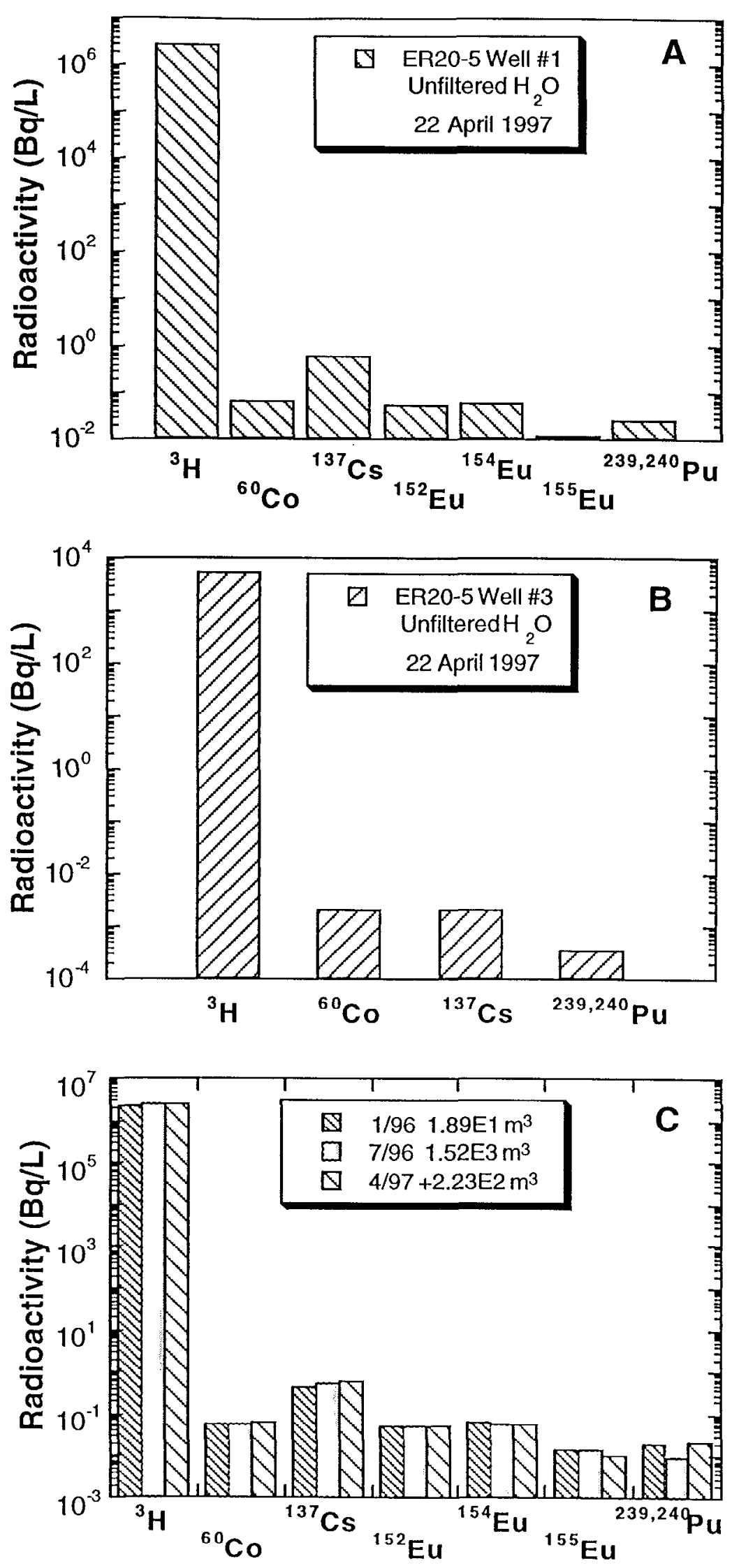

Figure 2 

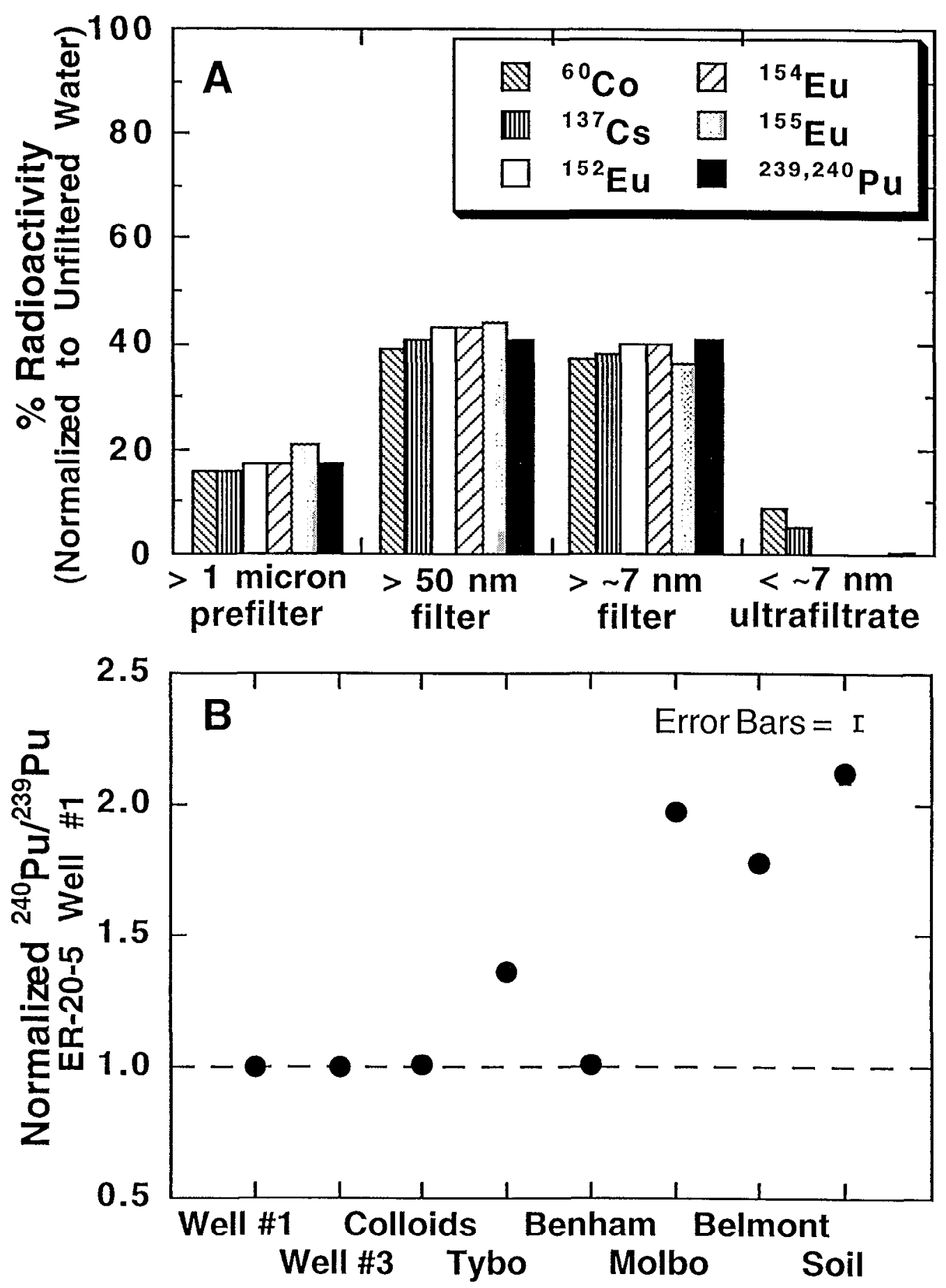

Figure 3 

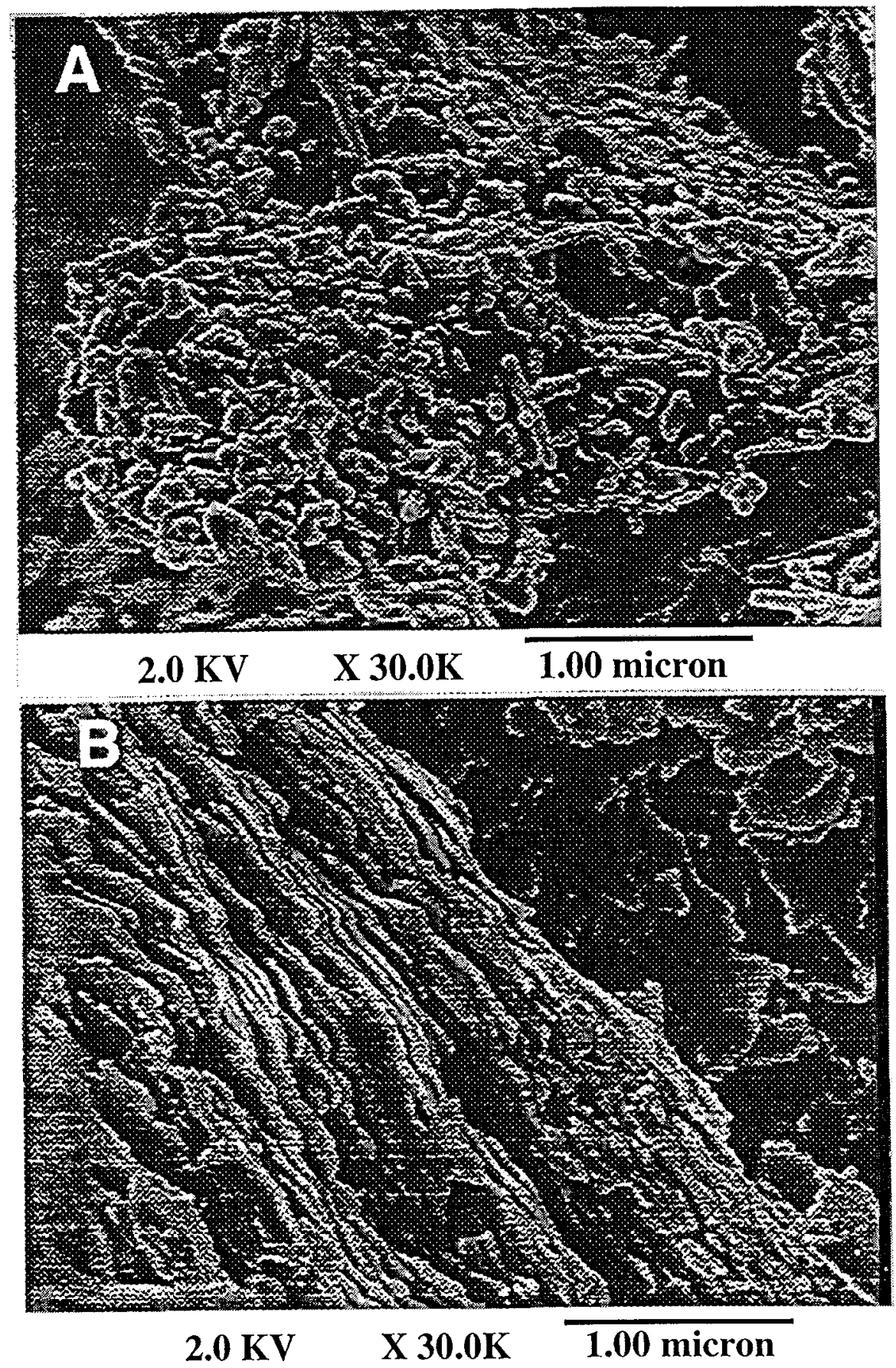

Figure 4 


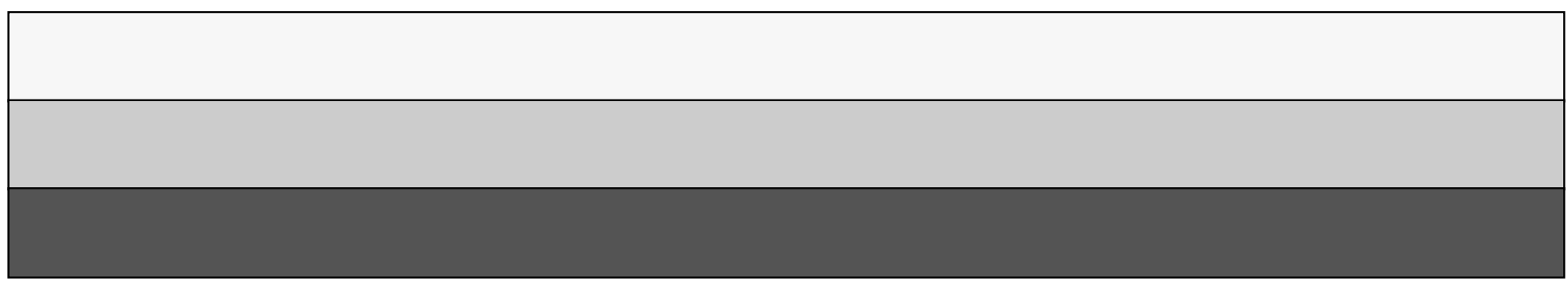

